
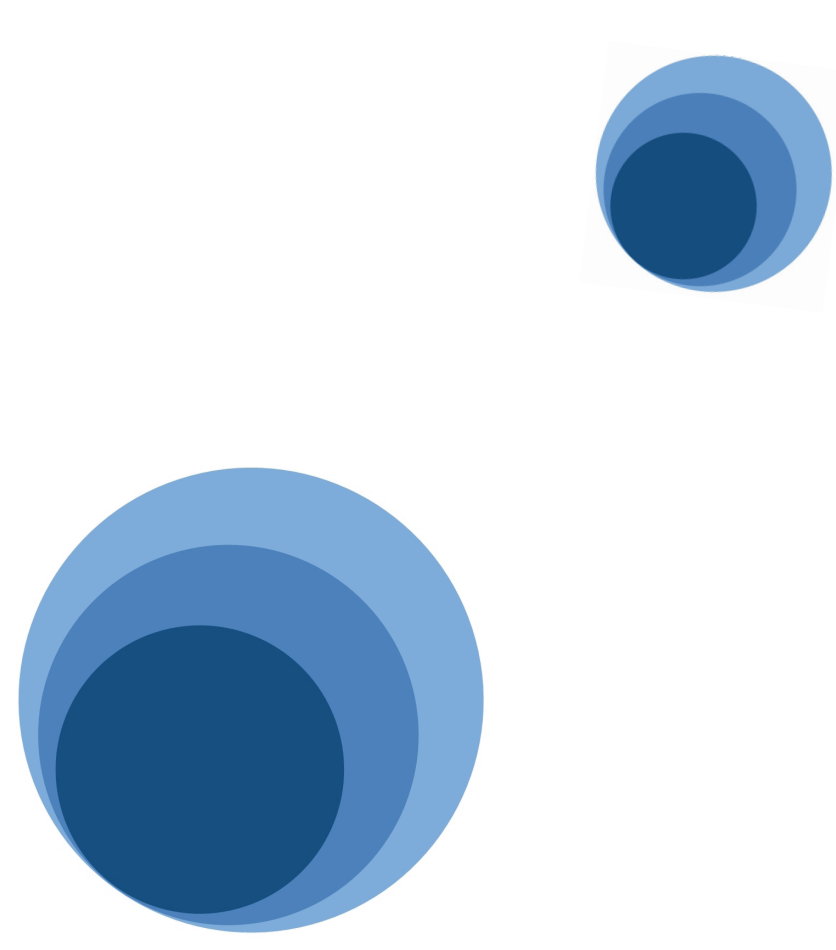

\section{INVITATION}

With immense pleasure, I would like to invite you to the public defense of my doctoral thesis, to be held on

Wednesday, the 12th of May 2010 at 14:45 hrs

Location:

Building Waaier, University of

Twente, Enschede, the Netherlands

A reception will follow the ceremony at the same venue

Ashok Sridhar +31-649312024

Paranymphs:

Pelagia Glampedaki P.Glampedaki@utwente.nl

Dimitris Christoulis D.Christoulis@utwente.nl 
AN INKJET PRINTING-BASED PROCESS CHAIN FOR CONDUCTIVE STRUCTURES ON PRINTED CIRCUIT BOARD MATERIALS 


\section{Committee members:}

\section{Chairman}

Prof. Dr. F. Eising

Promoter

Prof. Dr. R. Akkerman

Assistant Promoter

Dr. D.J. van Dijk

Members

Prof. Dr. R.R. Baumann (Chemnitz University of Technology)

Prof. Dr. R. Benedictus (Technical University of Delft)

Prof. Dr. D.J. Schipper (University of Twente)

Prof. Dr. M.M.C.G. Warmoeskerken (University of Twente)

Dr. R. Legtenberg (Thales Nederland B.V.)

Dr. J. Perelaer (Friedrich-Schiller-University Jena)

\section{AN INKJET PRINTING-BASED PROCESS CHAIN FOR CONDUCTIVE STRUCTURES ON} PRINTED CIRCUIT BOARD MATERIALS/ Copyright (C) Ashok Sridhar, 2010.

The research described in this thesis was carried out at the Production Technology Group, Faculty of Engineering Technology, University of Twente, P.O. Box 217, 7500AE Enschede, the Netherlands.

The research described in this thesis was done under the framework of the PACMAN project, supported by the Dutch Ministry of Economic Affairs.

Front cover design by Ashok Sridhar, inspired by Microsoft Office Word 2007.

Printed by: Ipskamp Drukkers B.V., Enschede, the Netherlands.

ISBN: 978-90-365-3027-9

DOI: $10.3990 / 1.9789036530279$ 


\title{
AN INKJET PRINTING-BASED PROCESS CHAIN FOR CONDUCTIVE STRUCTURES ON PRINTED CIRCUIT BOARD MATERIALS
}

\author{
DISSERTATION \\ to obtain \\ the degree of doctor at the University of Twente, \\ on the authority of the rector magnificus, \\ prof. dr. H. Brinksma, \\ on account of the decision of the graduation committee, \\ to be publicly defended \\ on Wednesday, 12 May 2010 at 15:00 hrs.
}

by

\author{
Ashok Sridhar \\ born on 02 May 1980 \\ in Mayiladuthurai, India
}


This thesis has been approved by the promoter:

Prof. Dr. R. Akkerman

and

by the assistant promoter:

Dr. D.J. van Dijk 


\section{Table of contents}

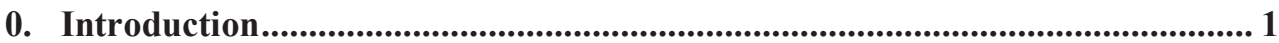

$0.1 \quad$ In the scheme of things ................................................................................................. 3

0.2 Goals and objectives of this thesis ....................................................................... 3

0.3 Structure of this thesis ............................................................................................. 4

1. Inkjet printing of functional inks and its associated pre- and postprocessing techniques................................................................................................................... 7

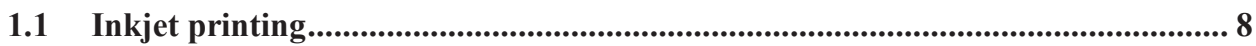

1.1.1 Inkjet printing techniques......................................................................... 8

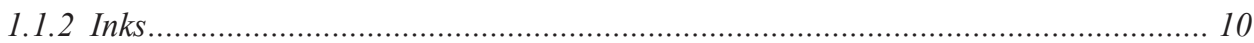

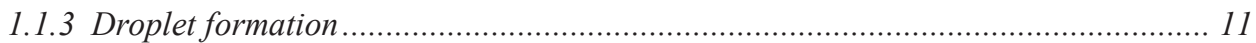

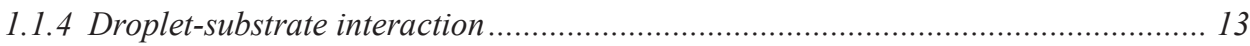

1.2 Pre-print-processing of substrates using surface modification techniques......... 16

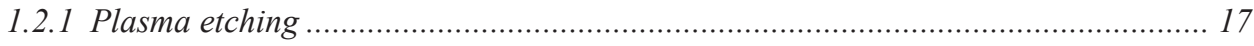

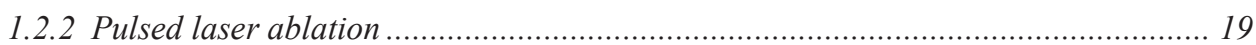

1.3 Post-print-processing of substrates ......................................................... 20

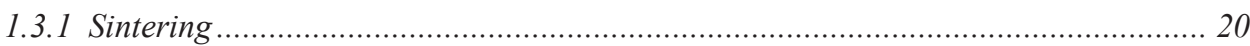

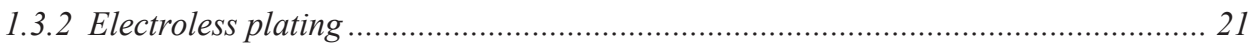

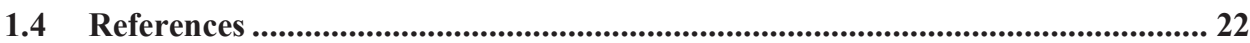

2. Fabrication of functional 3D structures by inkjet printing .............................. 27

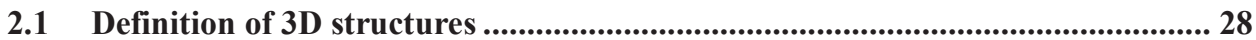

2.2 Fabrication of continuous structures from droplets.......................................... 28

2.3 Layer build-up using inkjet printing.......................................................... 31

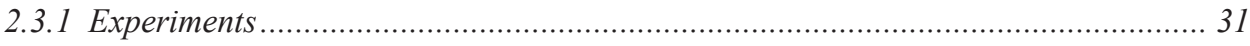

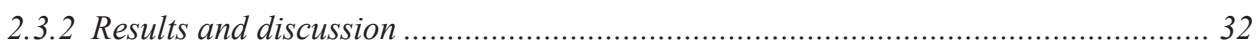

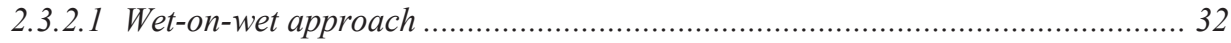

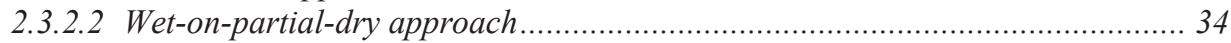

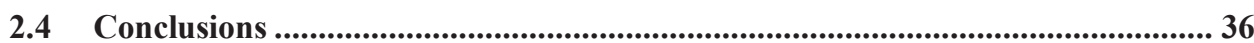

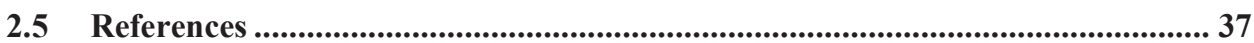

3. Inkjet printing of conductive structures on a PCB material............................ 39

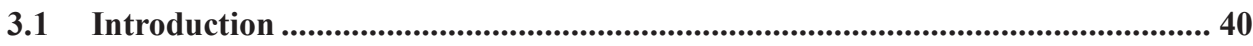

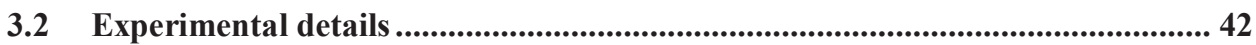




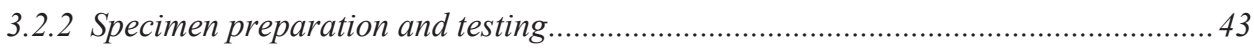

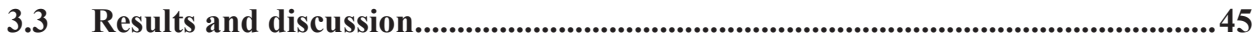

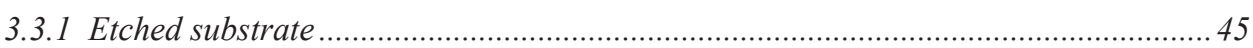

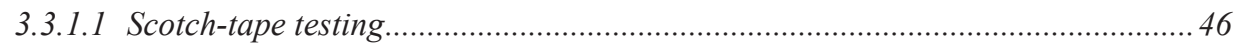

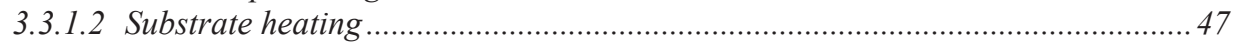

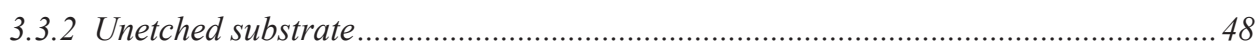

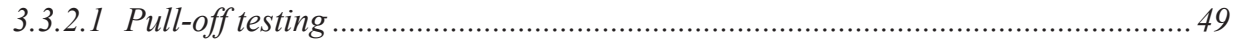

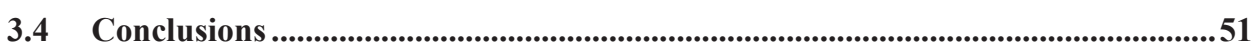

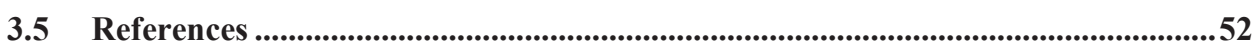

4. Plasma pre-treatment for inkjet printing- and electroless plating-based fabrication of conductive circuit structures ...........................................................55

4.1 Introduction ......................................................................................................................56

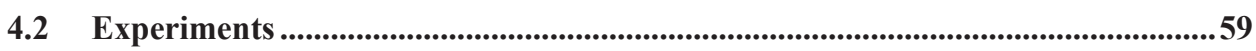

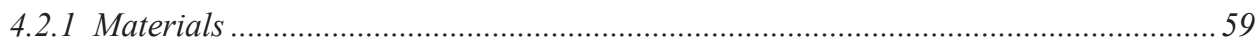

4.2.2 Initial inkjet printing-electroless plating trials without plasma treatment .................59

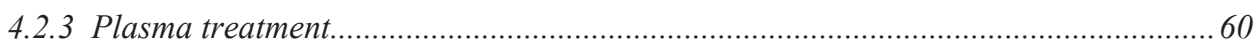

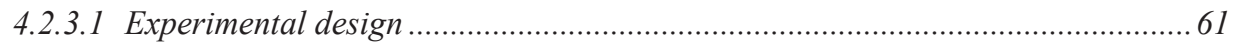

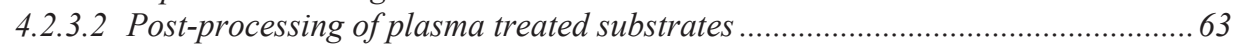

4.2.4 Inkjet printing-electroless plating trials after plasma treatment ............................... 65

4.3 Results and discussion...........................................................................................65

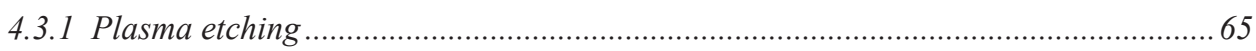

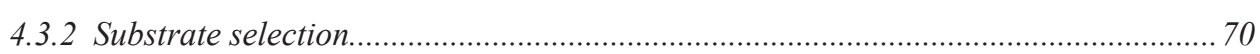

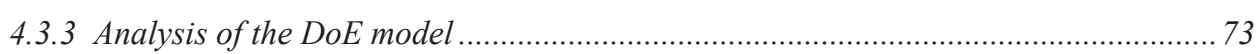

4.3.4 Electroless plating trials on plasma treated substrates .............................................. 79

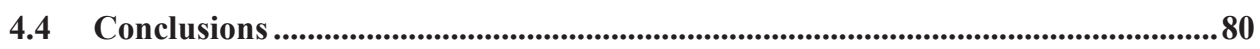

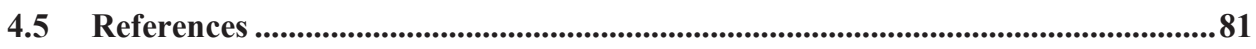

5. Quantification of adhesion in an inkjet printed metal-polymer system...........85

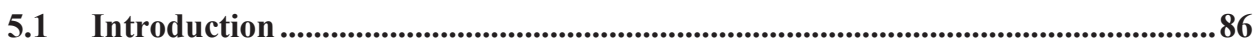

5.2 Important mechanisms of metal-polymer adhesion...............................................88

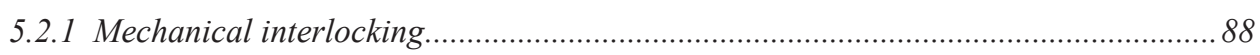

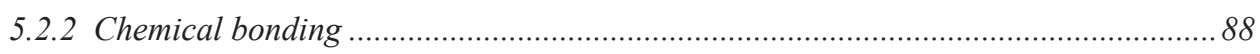

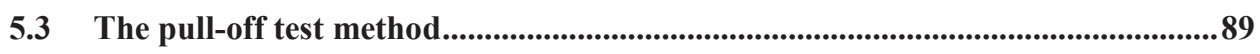

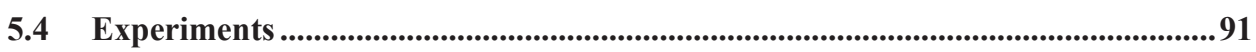

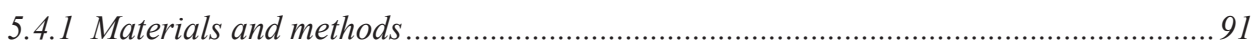




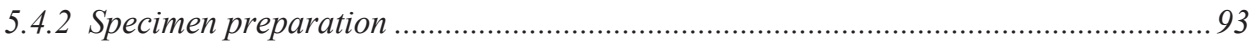

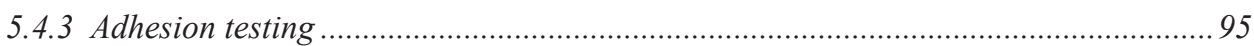

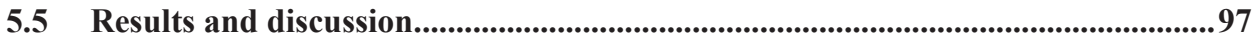

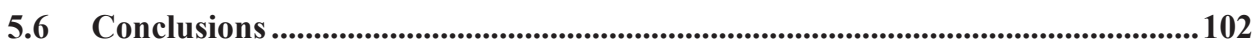

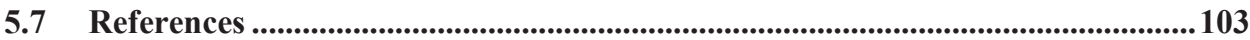

6. Fabrication of RF circuit structures using inkjet printing-electroless plating: a proof-of-concept ............................................................................105

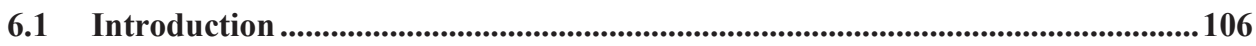

6.1.1 An inkjet printing-based process chain ................................................................... 106

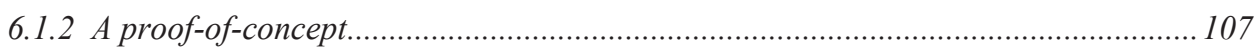

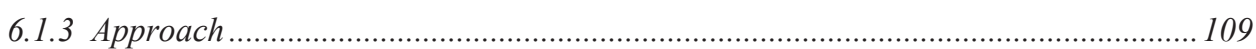

6.2 Filter design .................................................................................................................. 109

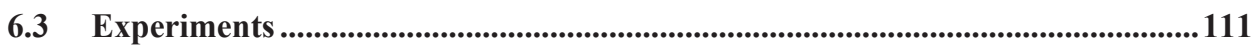

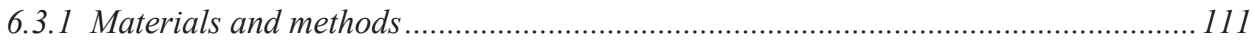

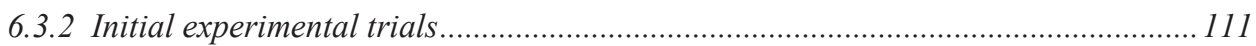

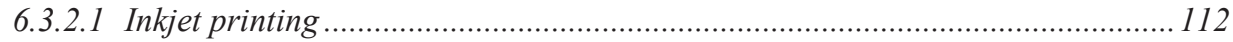

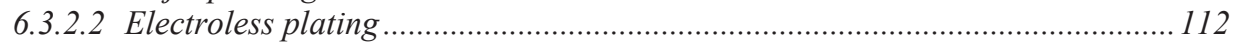

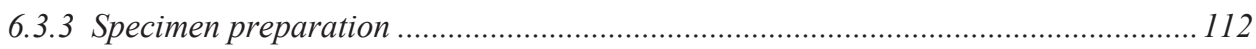

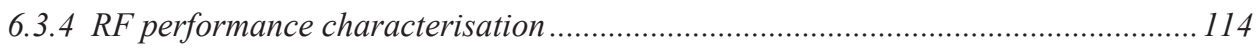

6.3.5 Reproducibility of the proposed fabrication method ............................................. 116

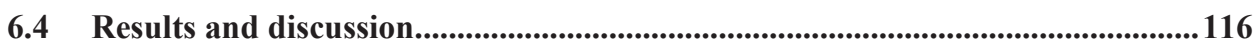

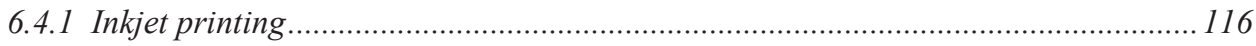

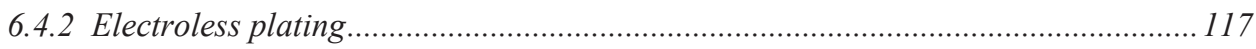

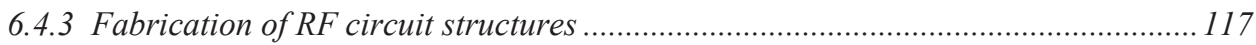

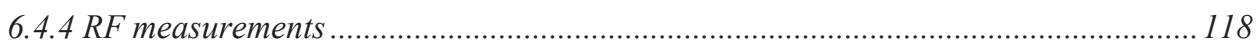

6.4.4.1 Comparison of printed-plated and reference circuit structures ........................ 118

6.4.4.2 Reproducibility of the proposed fabrication method ........................................... 121

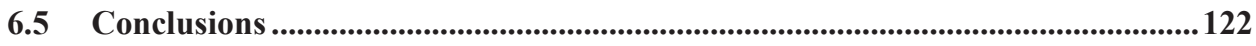

6.5.1 Reduction of manufacturing time and process scalability ......................................... 123

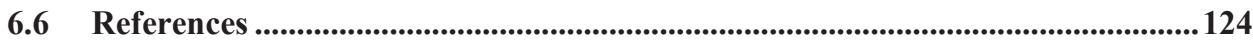

7. Conclusions and recommendations .....................................................127

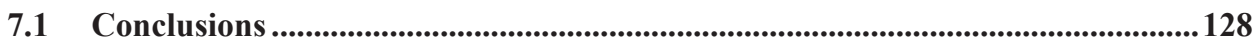

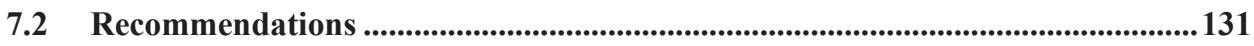

Appendix-A .....................................................................................................................133 


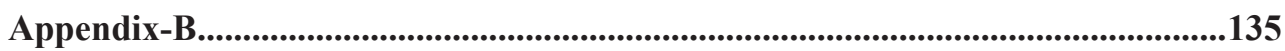

Nomenclature .................................................................................................................................139

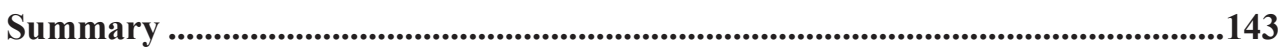

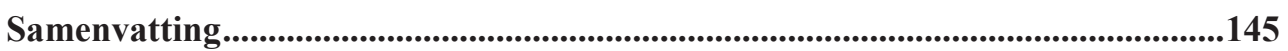

Acknowledgements ................................................................................................................147 
0. Introduction 
Chapter 0

This doctoral research was carried out under the framework of the PACMAN project funded by the Dutch Ministry of Economic Affairs. PACMAN is the abbreviation of Phased Array Communication antennas for Mass-market Application Needs. This project was initiated with the objective of developing a cluster of techniques that will enable the design, packaging and mass-manufacturing of low-cost phased-array antenna systems. Thales, ASTRON (The Netherlands Institute of Radio Astronomy), University of Twente and Eindhoven University of Technology were the collaborators in this project, which concluded in December 2008.

The research that culminated in this thesis was classified under a module of the PACMAN project, titled 'Multi-functional enabling technologies'. The intention of this module was to identify an appropriate fabrication method that has the capability to realise multiple electronics-related functionalities in an additive manner. Inkjet printing was singled-out as the key enabler for this purpose due to the following reasons:

- It is a non-contact process. Hence, it is theoretically possible to deposit material on any kind of surface irrespective of its composition and morphology.

- It is a free-form fabrication technique i.e. using inkjet printing, it is possible to generate $3 \mathrm{D}$ structures without the aid of additional jigs, fixtures and moulds. The structures to be fabricated can be designed and directly fed to the software program that controls the printhead's movements as well as droplet firing. This offers unlimited design and packaging freedom.

- Since inkjet printing is an additive process, electronic structures can be 'generated' instead of 'machined' or 'etched'. This potentially eliminates many process steps and minimises material wastage.

- By the time this research work began, many research institutions, universities and companies were already actively developing new 'functional inks' (chapter 1 presents a detailed account on such inks). Hence, the signs that inkjet printing is a suitable technique for electronics fabrication were indeed encouraging. 


\subsection{In the scheme of things}

In the context of printed electronics, inkjet printing is seen as a key enabler in fulfilling hitherto unrealised packaging possibilities and freedom of fabrication. At the start of this research project in 2006, several research institutions, universities and companies were already actively pursuing ways to identify potential application areas for inkjet printing, besides working on introducing it either as a substitute or as a complementary technique to existing fabrication methods. There was a striking similarity to most of these research initiatives: the use of flexible carrier substrates. These substrates are suitable for what is known as reel-to-reel or roll-to-roll printing, which encompasses a broad application spectrum ranging from radio frequency identification devices (RFID) to high-frequency antenna structures for radio astronomy. The sheer volume of flexible circuits required for various applications justifies the selection of flexible substrates for inkjet-oriented research. Apart from the flexible substrates, glass is also widely used for investigations on inkjet printing of organic light emitting diodes (OLED).

Rigid polymer substrates with E-glass fibre reinforcements are required in applications where robustness of the support structure i.e. carrier substrate is vital. Moreover, thick polymer substrates are needed to meet the dielectric requirements of numerous electronics applications. However, even today, there is hardly any research focussing on inkjet printing of functional structures on rigid, reinforced polymer-based substrates. For this reason, it was decided to select such substrates for this research. This brings in additional factors to be considered such as rougher surface topography when compared to thin, flexible substrates, and crimping of the polymer matrix around the E-glass fibres, resulting in surface undulations. The influence of these factors and how they were minimised/compensated constitute an important part of this thesis.

\subsection{Goals and objectives of this thesis}

The principal objective of this thesis is to demonstrate that an inkjet printing-based process chain can be successfully applied to realise radio frequency (RF) electronic functionalities on printed circuit board (PCB) materials. The term 'process chain' is used here to indicate that apart from inkjet printing, all the pre-inkjet printing- and post- 
Chapter 0

inkjet printing-processing steps have also been taken into consideration and studied in detail. Pre-inkjet printing processing improves the adhesion between the substrate and the printed structures; post-inkjet printing processing improves the electrical conductivity of the printed structures and improves their robustness. To accomplish this principal objective, the following goals and objectives were set at the beginning of this research:

- To identify a suitable functional ink and an inkjet printing system that will enable the realisation of electronic circuit structures.

- To analyse the geometrical aspects of inkjet printed structures.

- To ascertain the influence of the surface morphology of the substrate on inkjet deposited inks, based on experimental trials using the chosen functional ink. As mentioned before, 'substrate' refers to rigid, reinforced polymeric carriers.

- To identify a suitable technique to optimise the surface morphology of a substrate in order to make it favourable to inkjet printing, besides improving the adhesion between inkjet printed structures and substrates. The application of a systematic optimisation strategy was desired.

- To qualitatively and quantitatively determine the adhesion of inkjet printed structures to the substrate, making use of existing test methods either as such or with necessary modifications.

- To inkjet print a functional radio frequency electronic circuit structure on a functional substrate and to characterise its electronic performance, as a proof-ofconcept.

\subsection{Structure of this thesis}

This thesis report is the summary of the work done over a period of four years. During this period, peer-reviewed technical papers materialised as a result of this research. The contents of these papers have been extensively used in this report, with some modifications. An attempt has been made to present them in a logical sequence. Each chapter is, by and large, self-sufficient and self-explanatory. However, they are not totally mutually exclusive - there is some overlap to allow for better readability and understanding. The gist of the individual chapters are summarised below: 
- Chapter 1 explains the fundamental aspects of inkjet printing. This includes discussions on various inkjet techniques, inks, droplet formation and dropletsubstrate interaction, as well as pre- and post-processing of substrates.

- Chapter 2 is an account of fabrication of 3D structures using inkjet printing. 3D printing involves printing of structures that have more than one printed layer along their thickness. The different printing approaches followed to accomplish 3D material build-up are explained in detail.

- Chapter 3 deals with the issues arising from inkjet printing on commercially available rigid printed circuit board materials. It also sheds light on the problems in characterising the adhesion of structures printed on such substrates.

- Chapter 4 details an unconventional methodology to fabricate conductive structures on PCB substrates. This methodology involves a combination of inkjet printing and electroless plating. Due to poor adhesion, the surface morphology of the substrate had to be modified using tetrafluoromethane/oxygen $\left(\mathrm{CF}_{4} / \mathrm{O}_{2}\right)$ plasma treatment before fabricating the conductive structures. The plasma treatment-based optimisation of surface morphology of a high frequency substrate material widely used in the PCB industry, using a statistical design of experiments, is also explained in detail. The optimisation criteria were conduciveness to accurate inkjet printing and improved adhesion when compared to untreated substrate.

- Chapter 5 deals with the quantification of interfacial adhesion in an inkjet printed metal-polymeric substrate system. For this purpose, the pull-off test method was used. Additionally, an experimental methodology for segregating the principal adhesion components, namely chemical bonding and mechanical interlocking, is presented in this chapter.

- Chapter 6 deals with the proof-of-concept of the fabrication methodology presented in chapter 4. For this purpose, an S-band filter and a transmission line were inkjet printed-electroless plated on a high frequency substrate material, and subsequently characterised for their RF behaviour. The results of RF measurements were then compared with those of similar structures fabricated using the conventional lithography-based method. The comparative analysis and the associated RF measurements are presented in this chapter. 
Chapter 0

- Chapter 7 presents the conclusions of this research and recommendations concerning the directions for future research.

The components of this thesis report and their relationship are depicted in figure 0.1.

This figure shows the sequence in which the activities were carried out during this research. The proof-of-concept represents the validation of the proposed fabrication methodology.

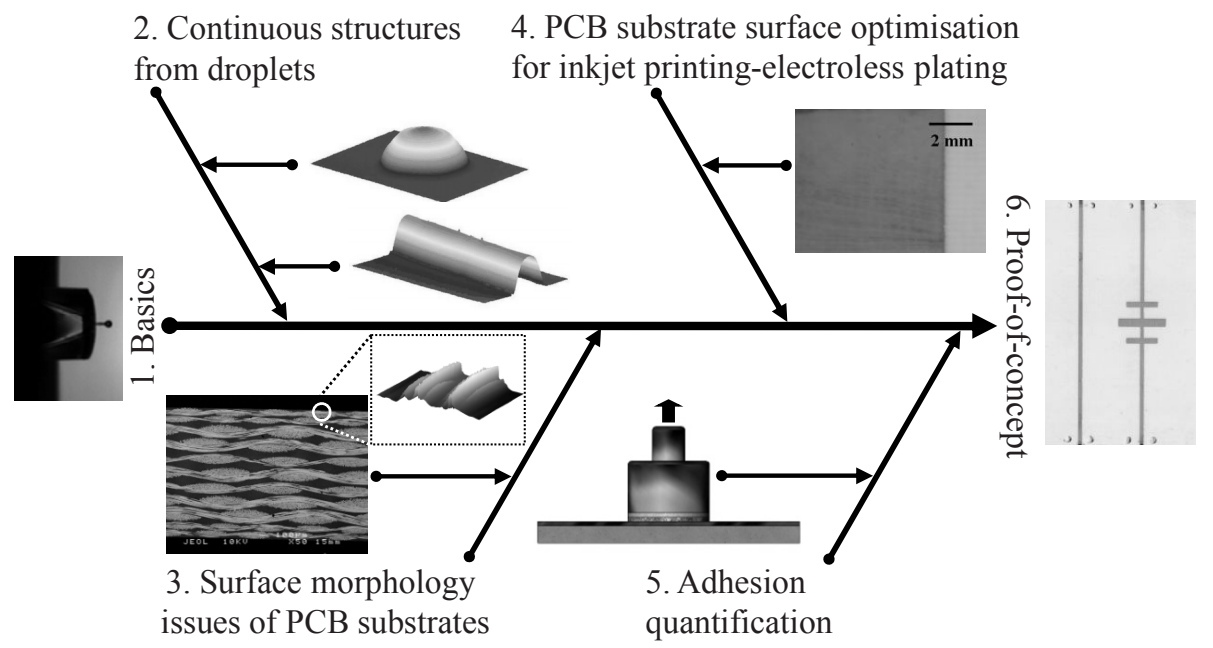

Figure 0.1: Connection between the individual chapters of this thesis; the numbers refer to the corresponding chapters. 


\title{
1. Inkjet printing of functional inks and its associated pre- and post-processing techniques
}

\begin{abstract}
In this chapter, inkjet printing of functional inks and the pre- and postprocessing techniques used in this research are discussed. Pre-processing steps are important to make the substrate suitable for printing as well as to improve the adhesion between the printed structure and the substrate. Postprocessing of the printed structures is needed in order for these structures to have physical continuity and to fulfil an intended functionality e.g. electrical conductivity. Although a wide range of pre- and post-processing techniques are available for different types of inks, substrates and functionalities, only those that are used during the course of this research are discussed in detail in this chapter.
\end{abstract}


Chapter 1

\subsection{Inkjet printing}

Inkjet printing is one of the most widely used digital printing techniques. From office printers to high-speed printing systems, it is a key technique for printing on paper. It is a non-contact printing technique that transfers ink directly onto the substrate. The ink is usually a liquid, although hot-melt inks that are liquefied just prior to printing are also used in some applications [1]. In the past decade, research initiatives and commercial applications beyond printing on paper have opened up inkjet printing to wider applications. One such application is the so-called 'printed electronics' or 'organic electronics'. Printed electronics refers to the application of various printing techniques like inkjet printing, screen printing, gravure printing etc to fabricate electronic components/circuits. The term organic electronics refers to the use of organic materials, e.g. conducting polymers, in electronics applications.

The advent of functional inks containing metal nanoparticles or conducting polymers, whose rheological properties fall within the scope of inkjet printing, has led to widespread research into its suitability to replace or complement existing electronics fabrication techniques. Using inkjet printing and functional inks, electronic circuit interconnections [2] and circuit components like resistors, capacitors and inductors [3] can be printed on a substrate. Even rectifying diodes and light emitting diodes (LED) [4,5] can be fabricated by this method. It should be mentioned though that the performance of semi-conducting inks presently available is not comparable to traditional semi-conducting materials like silicon. Inkjet printed electronics is an evolving field and still a lot of research needs to be done to develop functional inks that encompass the whole spectrum of electronics fabrication in an effective and efficient manner. Throughout this thesis, inkjet printing is dealt with as a tool to realise printed electronics only. Other applications of inkjet printing e.g. chemical and biological are not discussed. Moreover, only the application of metallic inks is discussed.

\subsubsection{Inkjet printing techniques}

Continuous inkjet printing and drop-on-demand inkjet printing are the two major classifications of this technology. As the name indicates, the former technique involves 
continuous ejection of droplets, while the latter prints only on demand i.e. only if the information to be printed requires them [1]. In continuous inkjet process, only part of the continuously generated ink droplets is directed onto the substrate in accordance with the pattern to be printed; the rest of the droplets are directed to a reservoir and are subsequently recycled. The hardware required to fulfil all the functionalities leading to successful continuous inkjet printing is much more complicated than that required for drop-on-demand inkjet printing. Mainly due to this reason, many contemporary research initiatives [6-9] make use of the drop-on-demand variant of inkjet printing. But in terms of printing speed, continuous inkjet printing is superior to the drop-on-demand variant. Continuous inkjet printing systems that operate at $500 \mathrm{kHz}$ are in existence [6].

Drop-on-demand inkjet printing is further classified into thermal inkjet (or bubble jet), piezo inkjet and electrostatic inkjet, based on the actuation methods deployed for droplet formation from the printhead of an inkjet printer [1]. Simplified representations of these three variants of drop-on-demand inkjet printing are shown in figure 1. In a thermal inkjet printhead (figure 1A), the ink droplet is squeezed out from the nozzle by the formation of a bubble, which is formed by heating the ink until it vaporises. In a piezo-actuated inkjet printhead (figure 1B), the ink droplet is ejected from the nozzle as a result of the pressure wave generated and propagated through the ink channel by the expansion-contraction cycle of the piezo element. This process is explained in detail in section 1.1.3. In the case of the electrostatic inkjet (figure 1C), the droplet is created due to an electric field that exists between the inkjet setup and the substrate. The droplet formation process involves a complex interaction of the surface tension ratio between the ink and the nozzle, and the electric field. Depending on the signal fed to the printhead, these forces can either be balanced or adjusted in such a way that a droplet is released [1].

Of these 3 variants of drop-on-demand inkjet printing, the piezo inkjet was chosen for this research due to its ready commercial availability and the following advantages it offers over the other two types:

a. Unlike the thermal inkjet, the ink need not be heated to produce droplets in a piezo-based system. This prevents degradation of functional materials present in the ink [10]. In short, piezo inkjet is an isothermal process. 
b. Due to the abovementioned reason, the range of solvents present in the ink that could be used in a piezo inkjet is broader than the thermal inkjet.

c. The investment and running costs needed for an electrostatic inkjet is much higher than a piezo inkjet. Moreover, this technique is still under development and is not as complete as either the piezo inkjet or the thermal inkjet in its evolution [1].
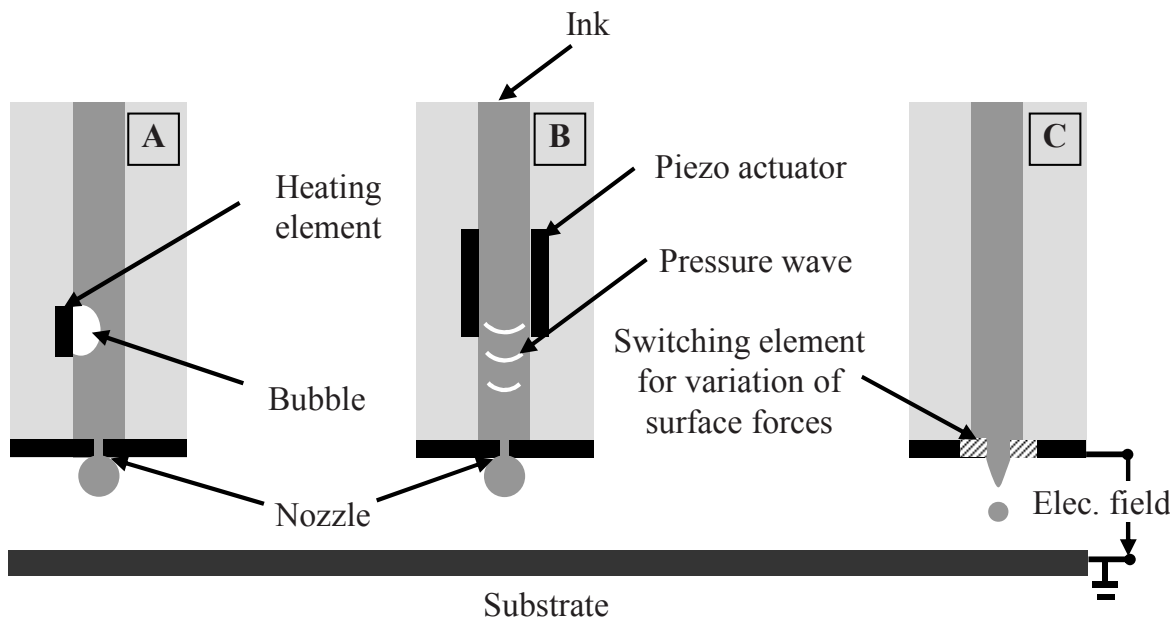

Figure 1.1: Drop-on-demand inkjet printing techniques [1]: (A) thermal inkjet or bubblejet, $(B)$ piezo inkjet and $(C)$ electrostatic inkjet.

\subsubsection{Inks}

Conventionally, majority of inks used in home and office inkjet printers are waterbased, and are dye-like or pigment-like. In the context of printed electronics, an ink contains functional material(s) that are organic or inorganic in nature. They are either dissolved (dye-like) or dispersed (pigment-like) in one or more solvents [9]. The role of the solvent is to provide a vehicle by means of which the functional material could be carried through the printhead and ejected via the nozzle. The functional material fulfils an electronic functionality. For example, conducting circuit interconnections can be fabricated by printing metal nanoparticles or organo-metallic complexes [11,12], and conducting polymers like Poly(3,4-ethylenedioxythiophene) poly(styrenesulfonate) (PEDOT:PSS) can be used as the hole-injecting material to fabricate OLEDs $[13,14]$. 
In this research, two types of metallic inks were used: (1) a silver nanoparticle-based dispersion [15], and (2) a soluble silver cluster and complex-based transparent solution [16], which can also be termed as an organic silver complex compound-based solution. While the former is a particle-based formulation that requires post-deposition heating in order to sinter the nanoparticles, the latter is a non-particle based precursor formulation that requires post-deposition heating to vaporise the organic component and to extract the metal. The mean nanoparticle diameter in the first ink type is about $5 \mathrm{~nm}$. Nanoparticles make up about $60 \%$ of the ink's weight and tetradecane is the solvent. The particles are prevented from agglomerating by dispersants.

The soluble silver cluster and complex-based ink consists of methanol and anisole as solvents, in addition to the organic silver complex, which makes up less than $77 \%$ of the ink, corresponding to a silver constituent of $15 \%$ by weight [16].

\subsubsection{Droplet formation}

Droplet formation in a piezo inkjet is dependant on a host of parameters. In the first place, the ink should be printable i.e. droplets should be produced from the ink. For this, the viscosity of the ink should be lower than $20 \mathrm{mPa}$.s. Otherwise, the energy carried by the pressure wave is viscously dissipated and no droplet is formed [17]. Surface tension of the ink is another key factor that determines droplet formation. Typically, it should be below $100 \mathrm{mN} \cdot \mathrm{m}^{-1}$. Otherwise, the piezo element should be supplied with high voltage to generate droplets [18]. However, these values are just guidelines; the actual range of these parameter values is different for different printhead makes. The viscosity and surface tension values of commonly used solvents such as water, methanol and tetradecane are well within the threshold values mentioned earlier. Figure 1.2 shows the droplet formation sequence in a piezo inkjet printer. The dispensed ink shown in this figure is PEDOT:PSS, and the nozzle diameter, $80 \mu \mathrm{m}$. 
Chapter 1

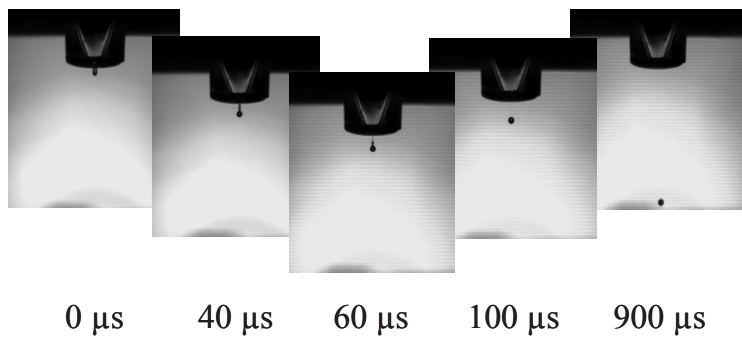

Figure 1.2: PEDOT:PSS droplet formation from a piezo drop-on-demand inkjet printer with an $80 \mu \mathrm{m}$ nozzle.

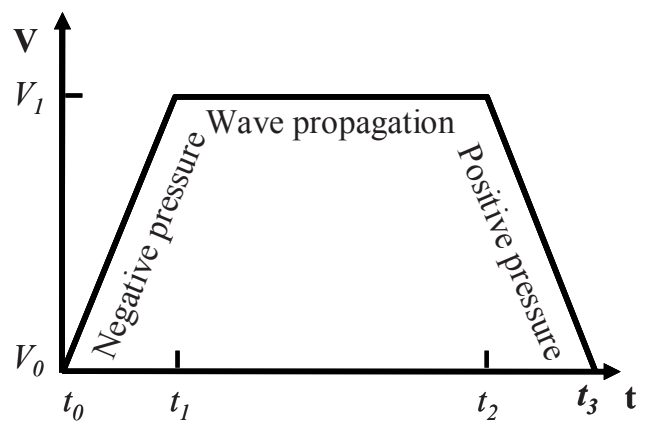

Figure 1.3: Trapezoidal pulse for piezo actuation.

As mentioned in section 1.1.1, the droplet formation is the result of the expansioncontraction cycle of the piezo element, which encloses a glass tube that contains an ink channel. This process can be explained with the example of a single trapezoidal unipolar voltage pulse shown in figure 1.3. In the timeframe $t_{0}$ to $t_{l}$ during which the voltage is ramped up from 0 to $V_{l}$ volts, the piezo element expands. This timeframe is in the order of a few $\mu \mathrm{s}$. Due to the sudden expansion of the piezo element, a negative pressure wave is set up in the channel. During the subsequent dwell stage i.e. timeframe $t_{1}$ to $t_{2}$, the pressure wave propagates inside the channel. This timeframe is usually in the order of a few tens of $\mu \mathrm{s}$. When it encounters the nozzle end, part of it is reflected. The reflected wave is superimposed by the positive pressure wave created due to the contraction of the piezo element, which in turn is caused by the voltage drop from $V_{l}$ to $V_{0}$ (timeframe $t_{2}$ to $t_{3}$ ). This leads to a constructive interference that overcomes the surface tension forces of the ink column and results in the formation of an ink jet $[6,19,20]$. If the wavelength of the pressure wave is greater than the diameter of the jet, the jet breaks up to form droplets. This effect is known as the 'Rayleigh-Tomotika 
instability' [17]. The jet is then retracted back into the nozzle and this cycle can be repeated with a frequency of up to $30 \mathrm{kHz}$ [6]. The wave parameters should be modulated in an ink-specific manner to obtain the proper break-off of droplets and to obtain optimal droplet formation without disturbances. The droplet size itself is determined by the voltage supplied to the piezo element and the nozzle diameter. The droplet volume is usually in the picolitre range.

The gap between the tip of the nozzle and the substrate is typically in the order of a few hundred micrometers. This gap is necessary to allow the droplet to stabilise itself after breaking off from the jet and to attain its equilibrium velocity [6]. However, if the gap is too high, any deviation of the droplet path from the straight line will be amplified and droplet positioning accuracy will be affected. Moreover, the larger the distance between the nozzle tip and the substrate, the longer the exposure of the droplet to atmospheric influences like temperature, humidity, convection currents due to substrate heating and draft due to the movement of the substrate holder. During the printing trials of this research, the gap was maintained between $0.5 \mathrm{~mm}$ and $2 \mathrm{~mm}$, determined on an empirical basis.

\subsubsection{Droplet-substrate interaction}

During its travel from the nozzle to the substrate, the solvent present in the droplet evaporates partially, the rate being dependant on the ambient temperature, the specific heat capacity and the vapour pressure of the solvent. For a given solvent, the percentage of solvent that evaporates in-flight depends on the nozzle tip-substrate distance and ambient conditions. After falling on a substrate, a droplet spreads due to the 'pull' of the substrate surface resulting from the latter's surface energy. While the surface tension of the droplet tends to keep its surface area as low as possible, the surface energy of the substrate tries to increase the substrate-droplet interfacial area in order to minimise its own free energy. The ability of a liquid to spread and wet a solid surface is given by the spreading coefficient $S_{S L}$. This coefficient is related to the surface free energy by the following equation [21]:

$$
S_{S L}=\gamma_{S V}-\gamma_{S L}-\gamma_{L V}
$$


Chapter 1

In this equation, $\gamma_{S V}$ is the surface free energy of the surface of the solid (J.m $\left.{ }^{-2}\right), \gamma_{S L}$ is the interfacial free energy between the solid and the liquid ( $\left.\mathrm{J}_{\mathrm{m}}^{-2}\right)$, and $\gamma_{L V}$ denotes the surface tension of the liquid $\left(\mathrm{N} . \mathrm{m}^{-1}\right)$. These physical quantities are represented in figure 1.4. If spreading of the liquid results in a decrease in interfacial free energy, then the spreading coefficient is positive. The larger the positive value of the spreading coefficient, the better the wetting behaviour, and consequently the spreading of the droplet on the surface of a solid. A negative value indicates poor wetting. Hence, if the solid has high surface free energy when compared to the surface tension of the liquid, and if the solid-liquid interfacial free energy is low (as a result of good affinity between the two), the liquid will show a greater propensity to wet the surface of the solid and consequently, the contact angle will be low.

Figure 1.4 shows the energy balance of droplet-solid surface interaction. Young's equation (eqn. 1.2) specifies the condition for a thermodynamic equilibrium of the three phases involved in this system, viz. solid, liquid (droplet) and vapour. The equilibrium contact angle is denoted by $\theta$.

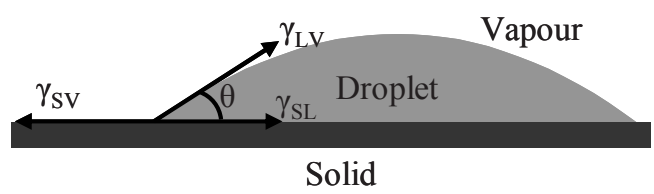

Figure 1.4: Energy balance for droplet-solid surface interaction.

$\gamma_{S L}-\gamma_{S V}+\gamma_{L V} \cos \theta=0$

Eqn. (1.2)

When an equilibrium state is reached and the droplet ceases to spread, its edge 'pins' itself and the solvent continues to evaporate. This is when the so-called 'Coffee-drop' or 'Coffee-stain' effect comes into picture. These terminologies refer to the end-result of an evaporation gradient on the surface of the droplet. The influence of evaporation at the edge of a droplet is greater than that of its interior, resulting in the onset of a replenishing flow from the interior towards the edge i.e. the pinned contact line. This flow carries the solvent, functional material as well as other components of the ink towards the edge. The outcome of these evaporation and replenishing flow mechanisms 
is the increased concentration of the functional material at the edge. The coffee-drop or coffee-stain effect was discussed in detail by Perelaer [6].

All through this process, the fluid components of the ink i.e. solvent, dispersant and other additives interact with the substrate to form bonds. The nature of these bonds could be chemical, physical or electrostatic, depending on the chemical structure of these ink components. At this stage, the metallic component of the ink droplet is still in the form of either an organo-metallic complex or nanoparticles stabilised by the dispersant.

After the solvent and other additives like the dispersant vaporise completely, only silver nanoparticles or metallic silver remain on the substrate. Usually, the complete evaporation needs additional heat energy, which can be supplied in two different ways: (1) by heating the substrate during the printing process, which ensures, depending on the substrate temperature and the ink's boiling point, flash evaporation of the solvent; and (2) by sintering the printed structure, which is necessary to remove the dispersants as well as other additives, and to impart continuity to the metallic component of the ink. While heating breaks the bonds between the substrate and the non-metallic components of the ink, and subsequently decomposes the latter, the substrate-silver interface begins to form, aided by various bonding mechanisms like chemical, physical, electrostatic, along with mechanical interlocking. These mechanisms are responsible for the adhesion between the printed structure and the substrate. A detailed treatment of bonding mechanisms, adhesion and adhesion testing can be found in chapter 5 .

In this thesis, the following terminologies are used to refer to inkjet printed entities on a substrate: track, layer and structure. These terminologies are made clear by figure 1.5: 
Chapter 1

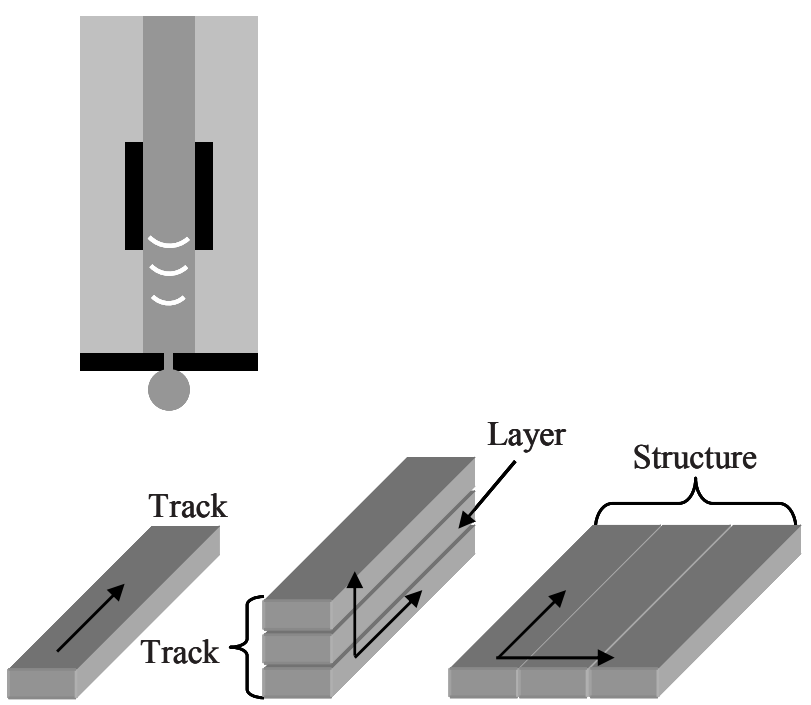

Figure 1.5: An illustration of the various terminologies associated with inkjet printed entities on a substrate; the arrows indicate the directions of material build-up by inkjet printing.

\subsection{Pre-print-processing of substrates using surface modification techniques}

Within the scope of this thesis, surface modification of substrates prior to printing was done to improve the interfacial adhesion between the inkjet printed structure and the substrate. Initial printing trials, as explained in chapters 2 and 3, were carried out without modifying the substrate surface. Adhesion testing using the Scotch tape test method qualitatively indicated that the interfacial strength was not high enough to ensure robust printed end- products. The reason for this is the fact that polymers, in general, possess low surface energy and are reluctant to form metal-polymer bonds [22, 23]. Another effect of the low surface energy is inferior wetting of the deposited ink when compared to, for instance, that on glass. Poor wetting results in high contact angles. Glass is perhaps the most commonly used substrate material in functional inkjet printing-related research. Poor wetting of the ink makes printing of multiple layers one on top of the other (i.e. stacking) difficult. Moreover silver, being a noble metal, is relatively unreactive when compared to non-noble metals [24]. Hence, mechanical interlocking between the substrate and the deposited metallic ink should be promoted by roughening the substrate. Since both wetting and adhesion are important for inkjet 
printed electronics, two surface modification techniques were deployed separately during the course of this research. They are plasma etching and pulsed laser ablation.

\subsubsection{Plasma etching}

Plasma is the fourth state of matter [25]. It is an ionised gas, in which both ions and free electrons are present. The ionisation is brought about by supplying energy to a gas (e.g. in the form of heat). The ionisation leads to radical formation, which in turn paves way for etching of materials [25].

A $\mathrm{CF}_{4} / \mathrm{O}_{2}$ plasma gas combination was chosen for the plasma trials carried out within the framework of this research, because the substrates used were polymer-based. Extensive literature is available that confirms the suitability of this gas combination to etch thermoset as well as thermoplastic polymers [26-28]. The chemical reactions are promoted by radicals in $\mathrm{O}_{2}$ and $\mathrm{CF}_{4}$. The key reactions leading to etching are as follows [28-31]:

(1) $\mathrm{CF}_{4}+\mathrm{e} \rightarrow \mathrm{CF}_{4}^{+} \rightarrow \mathrm{CF}_{3}^{+}+\mathrm{F}+2 \mathrm{e}$

(2) $\mathrm{O}_{2}+\mathrm{e} \rightarrow 2 \mathrm{O}+\mathrm{e}$

(3) $\mathbf{P}+\mathbf{F} \rightarrow \mathbf{P}^{\cdot}+\mathbf{H F} \uparrow$

(4) $\mathrm{P}+\mathrm{O} \rightarrow \mathrm{P}^{\cdot}+\mathrm{OH}$

(5) $\mathrm{P}^{\cdot}+\mathrm{O} \rightarrow \mathrm{P}^{\prime} \mathrm{O}+\mathrm{CO} \uparrow, \mathrm{CO}_{2} \uparrow$

(6) $\mathbf{P}^{\bullet}+\mathrm{F} \rightarrow \mathrm{PF}, \mathrm{PF}_{2}$

Here, $P$ represents the hydrocarbon molecule of the unmodified polymeric substrate, $P$ ' represents the polymer radical, and $P^{\prime}$ represents the final modified polymer structure, which contains $C=O$ formation. After its dissociation from the $\mathrm{CF}_{4}$ molecule, atomic fluorine (F) abstracts hydrogen atoms from the polymer, leading to the cleaving of carbon-carbon bonds. This creates radical sites on the polymer, which readily react with atomic oxygen $(\mathrm{O})$. This reaction results in the formation of carboxyl, carbonyl (both containing a $C=O)$ and hydroxyl $(\mathrm{OH})$ groups on the polymer surface [32], besides the volatile products mentioned in reaction (5). These groups enhance the hydrophilicity and the surface energy of the polymer, resulting in an enhanced tendency to form 
Chapter 1

chemical bonds with the inkjet deposited metallic ink, besides increasing the wettability. Moreover, the increased surface roughness of the substrate improves mechanical interlocking between them.

In this type of plasma etching, fluorine plays a catalytic role. However, if its concentration increases beyond an optimal level, passivation of the polymer surface occurs due to the formation of chemically stable fluorocarbon groups as in reaction (6) [28] and prevents etching by oxygen. Maximum etch rates occur at $\mathrm{CF}_{4}$ concentrations (in $\mathrm{CF}_{4} / \mathrm{O}_{2}$ plasma etching) between $10 \%$ and $25 \%$ of the gas mixture $[28,29]$, depending on the type of polymer. Hence, a substrate-specific optimisation of plasma treatment parameters is imperative before etching the substrates for improved adhesion.

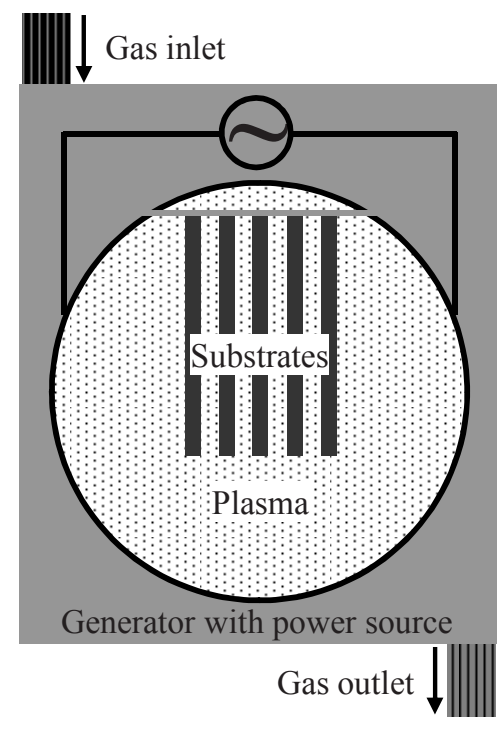

Figure 1.6: Schematic of a barrel plasma reactor.

There are different types of plasma reactors available, e.g. planar reactor, barrel reactor and downstream reactor. For this study, a barrel-type industrial-scale reactor was used. It is a tubular reactor in which the specimen to be etched is completely immersed in the plasma. An alternating current power source creates a magnetic field, which generates a voltage that sustains the plasma discharge. In this type of reactor, ion bombardment i.e. etching of the substrate by the accelerating ions plays little or no role [33]. Hence, 
chemical reactions similar to those already discussed are the dominant mechanisms of etching. This also means that the etching process is isotropic. The schematic of a barrel reactor is shown in figure 1.6.

\subsubsection{Pulsed laser ablation}

Pulsed laser etching was deployed to selectively pre-treat the substrate prior to inkjet printing, so that the mechanical interlocking between the deposited metallic ink and the substrate is enhanced. In this case, pre-treatment refers to patterning of the substrate by ablating holes, lines, hatches etc. Figure 1.7 shows polymeric substrates on which such patterns were ablated.
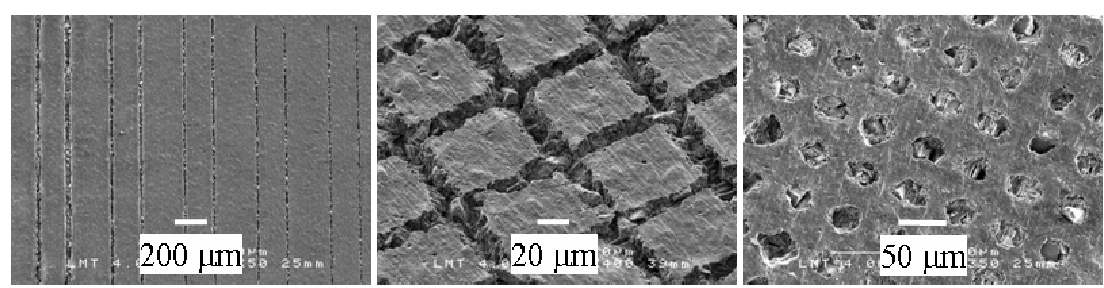

Figure 1.7: Different laser ablated patterns on a polymeric substrate (left to right): lines, hatches and holes.

The term 'ablation' means removal. The energy of the laser beam is transferred to the target material, resulting in material removal [34]. The high photon energy of the laser removes material by simultaneously inducing photolytic processes (e.g. bond scission) and pyrolytic processes (e.g. thermal degradation) [35]. A key factor to be taken into account before ablating a material is the 'ablation threshold'. It is a threshold beyond which the laser energy absorbed by the material is enough to cleave its molecular bonds. Polymers are well suited for laser pre-treatment of large areas due to their low ablation or material removal thresholds, which are typically lower than $200 \mathrm{~mJ} . \mathrm{cm}^{-2}$. Irradiation at fluences below the ablation threshold results in microstructural and chemical modification of the target material. In polymers, this may lead to reactions that lead to cross-linking, depolymerisation, induced radical reactions and photo-induced oxidation [35]. 
Chapter 1

The main advantage of using pulsed laser instead of continuous laser to ablate the polymeric substrate is that the heat diffusion into the substrate is minimal, due to the extremely short pulse duration [36]. The short pulse duration results in a very high instantaneous fluence which is laterally confined to the irradiated region [35]. This aids in precise patterning of the substrate with minimal thermal damage, due to reduced heataffected zone. In this research, a picosecond UV laser was used.

\subsection{Post-print-processing of substrates}

Subsequent to inkjet printing of metallic inks, the printed structures were subjected to two additional processes, namely sintering and electroless plating. While sintering is an integral step necessary to remove the non-metallic components of the deposited ink and impart continuity to the metallic structure, electroless plating is an optional step aimed at increasing the thickness of the inkjet-deposited metallic structures. It also offers additional advantages that are discussed in chapter 4 .

\subsubsection{Sintering}

As mentioned before, in a nanoparticle-based ink, dispersants prevent the nanoparticles from agglomeration. Nanoparticles have a lower melting point than the corresponding bulk metal due to the 'thermodynamic size effect' [37,38]. This effect arises from the increased surface-to-volume ratio of the nano-sized metal particles. However, in the context of this thesis as well as inkjet printed electronics, the term 'sintering' does not refer to melting of nanoparticles. Instead, it indicates a combination of particle growth and grain-boundary migration [39].

The most commonly used sintering technique is thermal sintering, in which the inkjet printed structure is cured in a convection oven or a hot plate. Other methods like microwave sintering and plasma sintering [6] have been experimented with, but they are still under development. Hence, thermal sintering was preferred for this study. The sintering temperature and time specified by the supplier [15] are $210^{\circ} \mathrm{C}$ and 60 minutes, respectively. The first stage of sintering is the removal of the solvent and dispersant from the deposited ink. At this stage, the nanoparticles still remain discrete [39]. 
Subsequently, as the heating continues, the particles start to coalesce and the grain size increases. The final state is reached when the deposited structure is continuous i.e. the grains have made sufficient contact to ensure continuity of the structure. However, the final structure is rarely $100 \%$ dense, as contaminants and pin holes often disturb the continuity of the sintered metal. Figure 1.8 depicts the mechanism of nanoparticle sintering [15]:

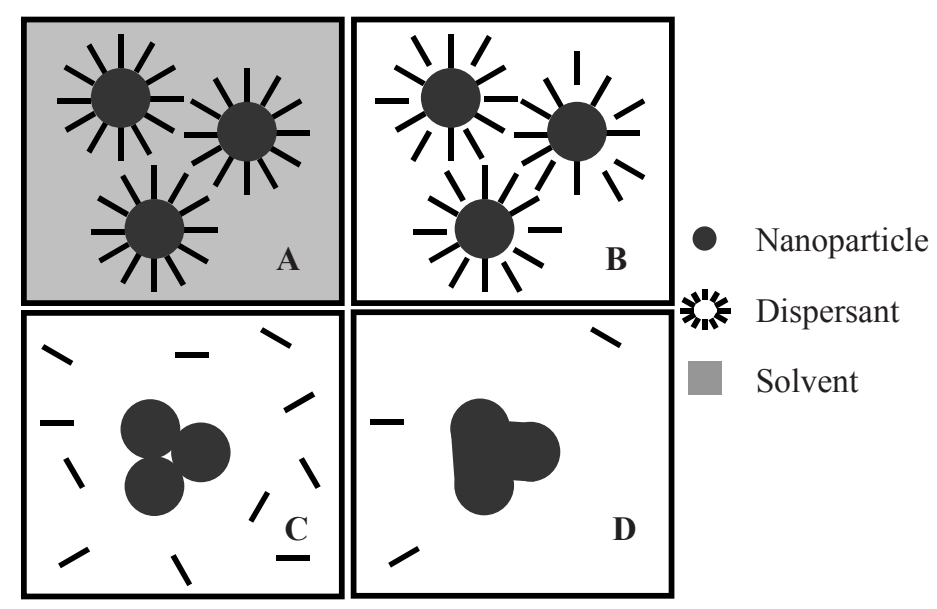

Figure 1.8: Stages in thermal sintering of nanoparticle: (A) before heating, (B) beginning of degradation by heat, $(C)$ sintering start, and $(D)$ sintering finish.

Globally speaking, the sintering mechanism of the other ink type i.e. organic silver complex compound-based solution is similar to that of the nanoparticle-based ink. However, owing to its different formulation, the specifics are different. Typically, this ink type needs lower sintering temperatures of about $150^{\circ} \mathrm{C}$, owing to the absence of surfactants. This enables the extracted metal particles to sinter more easily than in the case of nanoparticle-based inks [40].

\subsubsection{Electroless plating}

Electroless plating, as the name indicates, is a plating process that does not require an electric current. It is an auto-catalytic plating process, which is used to deposit metal layers on the target material, using a redox reaction. It is well suited for uniform metal deposition for a variety of substrates [41], including non-conducting ones. However, 
Chapter 1

when compared to electroplating, the metal deposition rate is lower [41], usually less than $5 \mu \mathrm{m} / \mathrm{hr}$ [42] for copper plating. Bath composition [43] and temperature [44] play a major role in determining the plating rate. This process is extensively used in the printed circuit board (PCB) industry $[41,45]$.

In electroless copper plating, copper ions in an alkaline solution are deposited on the target material due to their reduction. Formaldehyde is commonly used as the reducing agent. In the context of this research, the target material is the polymeric substrate on which silver seed layer is printed. The silver seed layer acts as the catalyst for this reaction. After the initial deposition, the reaction continues and more copper is deposited auto-catalytically on the freshly deposited copper [41]. The copper plating process can be expressed as the coupling of 2 partial electrochemical reactions [41]:

(1) $\mathrm{Cu}[\mathrm{L}]_{\mathrm{x}}{ }^{2+}+2 \mathrm{e}^{-} \rightarrow \mathrm{Cu}^{0}+\mathrm{xL}$

(2) $2 \mathrm{HCHO}+4 \mathrm{OH}^{-} \rightarrow 2 \mathrm{HCOO}^{-}+\mathrm{H}_{2} \uparrow+2 \mathrm{H}_{2} \mathrm{O}+2 \mathrm{e}^{-}$

In reaction (1), $L$ represents an organic ligand (known as 'chelating agent') that forms a stable $\mathrm{Cu}(\mathrm{II})$ complex. While reaction (1) is a cathodic copper reduction, reaction (2) is an anodic formaldehyde oxidation.

For this study, a commercially available electroless copper plating system [46] was used. It uses EDTA (ethylenediaminetetraacetic acid) as the chelating agent and, according to the supplier [46], has a high plating rate of 2 to $2.5 \mu \mathrm{m}$ per 30 minutes.

\subsection{References}

[1] H. Kipphan: "Handbook of print media", Springer-Verlag Berlin Heidelberg, 2001.

[2] T.H.J. van Osch, J. Perelaer, A.W.M. de Laat and U.S. Schubert: "Inkjet printing of narrow conductive tracks on untreated polymeric substrates", Advanced Materials, Vol. 20, No. 2, p. 343, 2008.

[3] D. Redinger, S. Molesa, S. Yin, R. Farschi and V. Subramanian: "An ink-jetdeposited passive component process for RFID", IEEE Transactions on Electron Devices, Vol. 51, No. 12, p. 1978, 2004. 
[4] Y. Yoshioka, P.D. Calvert and G.E. Jabbour: "Simple modification of sheet resistivity of conducting polymeric anodes via combinatorial ink-jet printing techniques", Macromolecular Rapid Communications, Vol. 26, No. 4, p. 238, 2005.

[5] Y. Liu and T. Cui: "Polymer-based rectifying diodes on a glass substrate fabricated by ink-jet printing", Macromolecular Rapid Communications, Vol. 26, No. 4, 289, 2005.

[6] J. Perelaer: "Microstructures prepared via inkjet printing and embossing techniques", Ph.D. Thesis - Eindhoven University of Technology, Eindhoven, the Netherlands, 2009.

[7] M. Mantysalo and P. Mansikkamaki: "An inkjet-deposited antenna for $2.4 \mathrm{GHz}$ applications", International Journal of Electronics and Communications, Vol. 63, p. 31, 2009.

[8] R. Kitsomboonloha, S. Baruah, M.T.Z. Myint, V. Subramanian and J. Dutta: "Selective growth of zinc oxide nanorods on inkjet printed seed patterns", Journal of Crystal Growth, Vol. 311, p. 2352, 2009.

[9] A. Kamyshny, M. Ben-Moshe, S. Aviezer and S. Magdassi: "Ink-jet printing of metallic nanoparticles and microemulsions", Macromolecular Rapid Communications, Vol. 26, p. 281, 2005.

[10] J. Sumerel, J. Lewis, A. Doraiswamy, L.F. Deravi, S.L. Sewell, A.E. Gerdon, D.W. Wright and R.J. Narayan: "Piezoelectric ink jet processing of materials for medical and biological applications", Biotechnology Journal, Vol. 1, No. 9, p. 976, 2006.

[11] A. Sridhar, J. Reiding, H. Adelaar, F. Achterhoek, D.J. Van Dijk and R. Akkerman: "Inkjet-printing- and electroless-plating-based fabrication of RF circuit structures on high-frequency substrates", Journal of Micromechanics and Microengineering, Vol. 19, No. 8, Doi:10.1088/0960-1317/19/8/085020, 2009.

[12] A. Sridhar, D.J. Van Dijk and R. Akkerman: "A comparative study of two conductive inkjet inks for fabrication of RF circuit structures", Proceedings of the LOPE-C, Frankfurt, Germany, 2009.

[13] W.-Y. Chou, S.-T. Lin, H.-L. Cheng, M.-H. Chang, H.-R. Guo, T.-C. Wen, Y.-S. Mai, J.-B. Horng, C.-W. Kuo, F.-C. Tang, C.-C. Liao and C.-L. Chiu: "Polymer lightemitting diodes with thermal inkjet printed poly $(3,4-$ ethylenedioxythiophene):polystyrenesulfonate as transparent anode", Thin Solid Films, Vol. 515, p. 3718, 2007. 
Chapter 1

[14] J. Wang, L. Sun, K. Mpoukouvalas, K. Lienkamp, I. Lieberwirth, B. Fassbender, E. Bonaccurso, G. Brunklaus, A. Muehlebach, T. Beierlein, R. Tilch, H.-J. Butt and G. Wegner: "Construction of redispersible polypyrrole core-shell nanoparticles for application in polymer electronics", Advanced Materials, Vol. 21, No. 10-11, p. 1137, 2009.

[15] Harima Silver Nanopaste (NPS-J) Datasheet, Harima Chemicals, Inc., Japan.

[16] InkTec TEC-IJ-040 Material Safety Datasheet, InkTec Co., Ltd., Korea.

[17] B.-J. De Gans, P.C. Duineveld and U.S. Schubert: "Inkjet printing of polymers: state-of-the-art and future developments", Advanced Materials, Vol. 16, No. 3, p. 203 , 2004.

[18] P.J. Smith: "Introduction to inkjet", Innovations in Inkjet - DPI Inkjet Workshop, Eindhoven, the Netherlands, 2007.

[19] H.-C. Wu, H.-J. Lin and W.-S. Hwang: "A numerical study of the effect of operating parameters on drop formation in a squeeze mode inkjet device", Modelling and Simulation in Material Science and Engineering, Vol. 13, No. 1, p. 17, 2005.

[20] D.-Y. Shin, P. Grassia and B. Derby: "Oscillatory limited compressible fluid flow induced by the radial motion of a thick-walled piezoelectric tube", Journal of Acoustical Society of America, Vol. 114, No. 3, p. 1314, 2003.

[21] J. Ge: "Interfacial adhesion in metal/polymer systems for electronics", Ph.D. Thesis - Helsinki University of Technology, Espoo, Finland, 2003.

[22] T.L. Gordon and M.E. Fakley: "The influence of elastic modulus on adhesion to thermoplastics and thermoset materials", International Journal of Adhesion \& Adhesives, Vol. 23, p. 95, 2003.

[23] C.T. Pan: "Selective electroless copper plating micro-coil assisted by $248 \mathrm{~nm}$ excimer laser”, Microelectronic Engineering, Vol. 71, No. 3-4, p. 242, 2004.

[24] L.-H. Lee: "Fundamentals of adhesion", Plenum Publishing Corporation, New York, USA, p. 71, 1991.

[25] D.C. Schram: "Is plasma unique? The presence of electrons and the importance of charge", Plasma Sources Science and Technology, Vol. 18, No. 1, Doi:10.1088/09630252/18/1/014003, 2009.

[26] G. Turban and M. Rapeaux: "Dry etching of polyimide in $\mathrm{O}_{2}-\mathrm{CF}_{4}$ and $\mathrm{O}_{2}-\mathrm{SF}_{6}$ plasmas", Journal of Electrochemical Society: Solid-state Science and Technology, Vol. 130, No. 11, p. 2231, 1983. 
[27] I. Garnev, K. Oshinov, V. Orlinov, K. Popova and B. Spangenberg: "Optimising plasma etching of base polymer materials for MLB using response surface methodology”, Bulgarian Journal of Physics, Vol. 19, No. 3-4, p. 74, 1992.

[28] A.M. Wrobel, B. Lamontagne and M.R. Wertheimer: "Large-area microwave and radiofrequency plasma etching of polymers", Plasma Chemistry and Plasma Processing, Vol. 8, No. 3, p. 315, 1988.

[29] S. Sapieha, A.M. Wrobel and M.R. Wertheimer: "Plasma-assisted etching of paper”, Plasma Chemistry and Plasma Processing, Vol. 8, No. 3, p. 331, 1988.

[30] M. Ikagawa, I. Tohno, T. Shinmura, S. Takagi, Y. Kataoka and M. Fujihara: "Study on surface modification of indium tin oxide and resist surfaces using $\mathrm{CF}_{4} / \mathrm{O}_{2}$ plasma for manufacturing organic light-emitting diodes by inkjet printing”, Japanese Journal of Applied Physics, Vol. 47, No. 12, p. 8935, 2008.

[31] C.J. Mogab, A.C. Adams and D.L. Flamm: "Plasma etching of $\mathrm{Si}$ and $\mathrm{SiO}_{2}$ - the effect of oxygen additions to $\mathrm{CF}_{4}$ plasmas", Journal of Applied Physics, Vol. 49, No. 7 , p. $3796,1978$.

[32] N. Inagaki: "Plasma surface modification and plasma polymerization", Technomic Publishing Company, Inc., USA, p. 78, 1996.

[33] S.M. Rossnagel, J.J. Cuomo and W.D. Westwood: "Handbook of plasma processing technology: fundamentals, etching, deposition, and surface interactions", Noyes Publications / William Andrew Publishing LLC, p. 18 - 20, 1990.

[34] Z. Zhang and Y. Liu: "Handbook of nanophase and nanostructured materials: volume 1", Kluwer Academic / Plenum Publishers, New York, USA, p. 219, 2003.

[35] H. Horn, S. Beil, D.A. Wesner, R. Weichenhain and E.W. Kreutz: "Excimer laser pretreatment and metallization of polymers", Nuclear Instruments and Methods in Physics Research B, Vol. 151, No. 1-4, p. 279, 1999.

[36] A.A. Serafetinides, M.I. Makropoulou, C.D. Skordoulis and A.K. Kar: "Ultra-short pulsed laser ablation of polymers", Applied Surface Science, Vol. 180, No. 1-2, p. 42, 2001.

[37] A.L. Stepanov, D.E. Hole, A.A. Bukharaev, P.D. Townsend and N.I. Nurgazizov: "Reduction of the size of the implanted silver nanoparticles in float glass during excimer laser annealing”, Applied Surface Science, Vol. 136, No. 4, p. 298, 1998. 
Chapter 1

[38] R. Garrigos, P. Cheyssac and R. Kofman: "Melting for lead particles of very small sizes; influence of surface phenomena", Zeitschrift fuer Physik D - Atoms, Molecules and Clusters, Vol. 12, No. 1-4, p. 497, 1989.

[39] V. Subramanian, S.K. Volkman and D.R. Redinger: "Printed zinc oxide based electronics: materials, devices, and outlook", Proceedings of the LOPE-C, Frankfurt, Germany, 2009.

[40] J. Perelaer, C.E. Hendriks, A.W.M. De Laat and U.S. Schubert: "One-step inkjet printing of conductive silver tracks on polymer substrates", Nanotechnology, Vol. 20, Doi: 10.1088/0957-4484/20/16/165303, 2009.

[41] J. Li and P.A. Kohl: "Complex chemistry \& the electroless copper plating process", Plating \& Surface Finishing, Vol. 91, No. 2, p. 40, 2004.

[42] C.F. Coombe: "Printed circuits handbook", The McGraw-Hill Companies, Inc., USA.

[43] G.O. Mallory and J.B. Hajdu: "Electroless plating fundamentals \& applications", Noyes Publications / William Andrew Publishing, LLC, New York, USA, p. 53, 1990.

[44] J.H. Lee, I.G. Lee, T. Kang, N.S. Kim and S.Y. Oh: "The effects of bath composition on the morphologies of electroless nickel under-bump metallurgy on $\mathrm{Al}$ input/output pad", Journal of Electronic Materials, Vol. 34, No. 1, p. 12, 2005.

[45] T. Ogura, M. Malcomson and Q. Fernando: "Mechanism of copper deposition in electroless plating”, Langmuir, Vol. 6, No. 11, p. 1709, 1990.

[46] Enthone Envision EC2130 Datasheet, Enthone Inc., USA. 


\section{Fabrication of functional 3D structures by inkjet printing}

This chapter is an account of experimental trials carried out to ascertain the possibility of using drop-on-demand inkjet printing to fabricate functional 3D structures. Since the main objective of this study is to evaluate the dimensional aspects of $3 \mathrm{D}$ inkjet printing and not the electrical or mechanical properties, glass was used as the substrate material. Three approaches were followed to print 3D structures: wet-on-wet, wet-on-dry and wet-on-partial-dry. The outcomes of these approaches were compared with the aid of optical microscopy and interferometry. This study is vital to understand the potential and limitations of fabricating 3D structures using inkjet printing. 
Chapter 2

\subsection{Definition of 3D structures}

A single inkjet printed layer is a few hundred nanometres thick. The exact thickness depends on the ink-type, substrate-type, printing parameters and the nozzle diameter. However, for most practical applications, including electronics applications, higher thicknesses are needed to fulfil the required functionalities. For example, a conducting structure with $5 \mu \mathrm{m}$ thickness may be needed for an RF circuit, so that the resistance to current flow is lowered (electrical resistance is inversely proportional to the thickness or cross-section of a conductor).

Technically speaking, even if five or ten layers are built-up i.e. stacked, the thickness is still negligible when compared to the other two dimensions of the printed structure, viz. length and width. Nevertheless, the term '3D structure' is used in this context to denote any structure that has more than one inkjet printed layer along its thickness. This implies that when the second layer is printed, the ink is not deposited directly on the substrate, but on the previous layer. This changes the surface and interface mechanisms quite drastically, as it involves metal-metal contact and bonding. In addition to that, the morphology of the previous layer is crucial in determining the droplet spreading behaviour, which means how the previous layer was deposited becomes important.

\subsection{Fabrication of continuous structures from droplets}

For the printing trials discussed in this chapter, an organic silver complex compoundbased solution (from InkTec Co. Ltd., Korea) was used as the ink. A drop-on-demand inkjet printer (Jetlab-4, from MicroFab Technologies Inc., USA) with an $80 \mu \mathrm{m}$ nozzle was used for all the printing trials of this study. The $\mathrm{X}$ and $\mathrm{Y}$ stages of the printer are capable of independent movements and they position the substrate holder. The $Z$ axis motion of the printer enables the adjustment of the height of the printhead that holds the nozzle. It is possible to heat up the substrate holder to $100{ }^{\circ} \mathrm{C}$. The same printer was used for all the printing trials that are mentioned in this thesis.

Before inkjet printing layer-upon-layer, single dots and single layers of silver were printed and analysed. Figure 2.1(A) shows a glass slide on which individual silver 
droplets were printed; figure 2.1(B) shows another glass slide on which a continuous track was printed. All the images in this chapter were recorded after sintering the ink, at $150^{\circ} \mathrm{C}$ for 30 minutes.
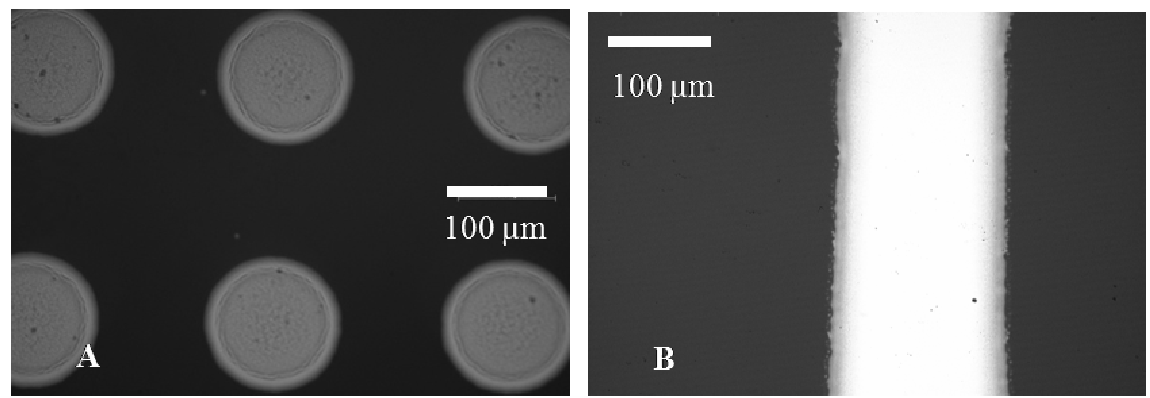

Figure 2.1: Inkjet printed $(A)$ single dots and $(B)$ single track on glass substrates.

To fabricate a continuous structure using inkjet printing, the first step is to understand the spreading behaviour of a droplet on the selected substrate material. Subsequently, the pitch i.e. centre-to-centre distance of two adjacent droplets should be determined. An arbitrary value for the pitch cannot be chosen; instead, it should be determined empirically to avoid dimensional irregularities. This is illustrated in figure 2.2 , which shows a single track (i.e. a structure printed by adding droplets in only one direction) printed with three different droplet pitches: $100 \mu \mathrm{m}, 110 \mu \mathrm{m}$ and $120 \mu \mathrm{m}$. The track printed with $100 \mu \mathrm{m}$ pitch is the best one of this lot. Scalloping of the edges of the tracks printed with the other two pitches can be observed from this figure. The cause for this is that the spacing between two adjacent droplets is too high, and hence a deposited droplet is unable to flow into the already generated track completely. If the spacing is reduced below $100 \mu \mathrm{m}$, bulging, which refers to the local lateral enlargement of the track, was observed. Similar observations were made by Perelaer [1] and Subramanian et al [2]. 
Chapter 2
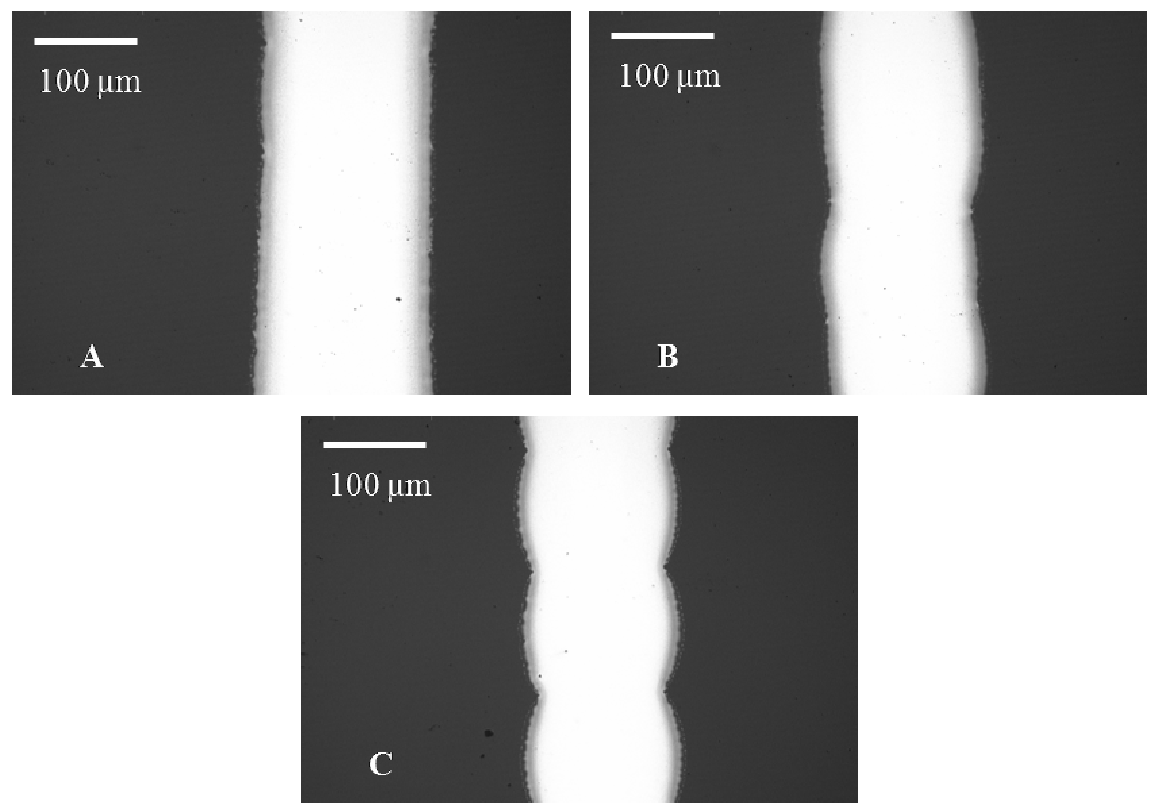

Figure 2.2: Inkjet printed single tracks on a glass substrate with $(A) 100 \mu \mathrm{m}$ droplet pitch, $(B)$ $110 \mu \mathrm{m}$ droplet pitch and (C) $120 \mu \mathrm{m}$ droplet pitch.

To print structures wider than a single track width, two different droplet pitches for $\mathrm{X}$ and $\mathrm{Y}$ directions of the $\mathrm{X}-\mathrm{Y}$ plane (i.e. plane of printing) should be optimised. This brings in an additional challenge: assuming that the width of a single printed track is $150 \mu \mathrm{m}$, if a track of $250 \mu \mathrm{m}$ width is needed, then the second track should be printed in such a way that it overlaps the first one by $50 \mu \mathrm{m}$. This gives rise to cross-sectional variations, which are not acceptable in some applications like high-radio-frequency electronics. There are ways to circumvent this problem, like e.g. local surface energy modification of the substrate. However, it should be done in a case-specific manner, and is time and cost intensive. 


\subsection{Layer build-up using inkjet printing}

\subsubsection{Experiments}

There are two ways of fabricating 3D microstructures using inkjet printing: (1) wet-onwet and (2) wet-on-dry. A comparison of these two approaches for functional printing has been done before for the case of screen printing [3]. Wet-on-wet printing involves printing a layer of ink without drying the previous layer i.e. the previous layer is still in a 'wet' condition. Wet-on-dry printing, in turn, could be done in two ways: (a) printing a layer after the previous one is moderately heated to remove the solvent and the additives (henceforth called 'wet-on-partial-dry') present in the ink, or (b) printing a layer after the previous one is sintered completely. In this research, only the wet-onpartial-dry approach along with wet-on-wet was followed. The approach in which the previous layer is completely sintered is, in most cases impractical, as the substrate has to be heated to the ink-specific sintering temperature (varies between $150^{\circ} \mathrm{C}$ to $210^{\circ} \mathrm{C}$ depending on the ink) for complete sintering. Since the tip of the nozzle is very close to the substrate, such high temperatures lead to modification of the ink behaviour and clogging of the nozzle due to the evaporation of the solvent inside the nozzle. Of course, sintering can be done externally, but very accurate repositioning of the substrate is required.

For the wet-on-partial-dry approach, the substrate temperature was maintained at $90^{\circ} \mathrm{C}$. Even in this case, the ink inside the nozzle heated up and the droplet size increased due to a reduction in viscosity, while printing large areas i.e. structures with large dimensions. Moreover, there were problems with droplet stability as well. These issues were addressed by moving the nozzle to a higher position every time after printing one layer. Sometimes, the ink had to be purged from the nozzle so that a small quantity of the ink close to the tip of the nozzle is ejected into a reservoir. This resulted in the unheated portion of the ink rushing in to replace the ejected ink. Obviously, purging is uneconomical, since the metallic inks are very expensive. There are other ways of solving the problem of the ink getting heated up. For instance, an adaptive cooling system integrated into the printhead can maintain the ink at a constant temperature, irrespective of the ambient and substrate temperature. One other approach was discussed in [1]: the gap between the tip of the nozzle and the substrate can be increased 
Chapter 2

while printing, so that the influence of the heat is reduced. However, it is not advisable to increase the gap beyond a few millimetres as external disturbances e.g. draft and vibrations begin to play a major role in droplet deposition accuracy. Hence, this approach has its limitations.

With a droplet pitch of $100 \mu \mathrm{m}$ in $\mathrm{X}$ and $\mathrm{Y}$ directions, multiple layers were printed on glass substrates in wet-on-wet and wet-on-partial-dry modes. Three stacked layers were printed in both cases and subsequently observed with an optical microscope and an interferometer. Surface roughness, track thickness and dimensional integrity of the track were the criteria studied. Based on the results of the analyses, up to five stacked layers were printed using the wet-on-partial-dry approach and analysed.

\subsubsection{Results and discussion}

\subsubsection{Wet-on-wet approach}

The structures printed using the wet-on-wet approach highlighted the following shortcomings: (1) there was no linear or quasi-linear increase in thickness of the printed tracks with increase in the number of layers, as shown in figure 2.3, and (2) the surface roughness was pronounced on thrice-printed structure as seen in figure 2.3(C). Such high roughness is unsuitable for most practical applications [4-6]. It was observed that the increase in track thickness in this case was less than the other (wet-on-partial-dry) approach. The high values associated with the legends of figure 2.3(B) and (C) are due to the surface roughness. The actual thicknesses of these tracks without considering the roughness peaks are much lower. Figure 2.3 also clearly shows that a single layer has a curved profile (cross-section), whereas the subsequent layers led to the flattening and widening of the cross-section. This can be attributed to the impact of a droplet on the previous undried one and the subsequent re-arranging influenced by the surface tension of the ink as well as the surface energy of the substrate. Figure 2.4 illustrates how the flattening occurs. It can be seen in this figure that when a second droplet is deposited on an undried preceding one, it leads to flattening and ripple formation. 

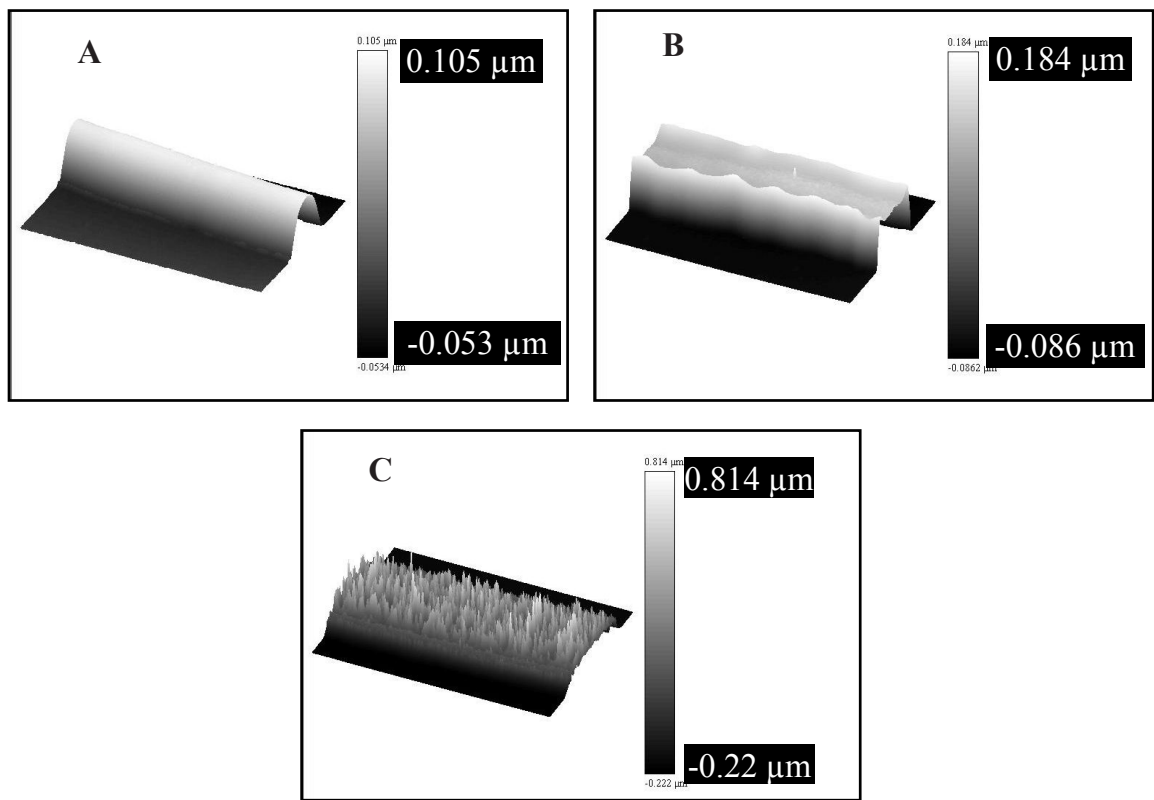

Figure 2.3: 3D interferometric images of inkjet printed tracks (wet-on-wet) on glass, containing $(A)$ one layer, $(B)$ two stacked layers and $(C)$ three stacked layers.
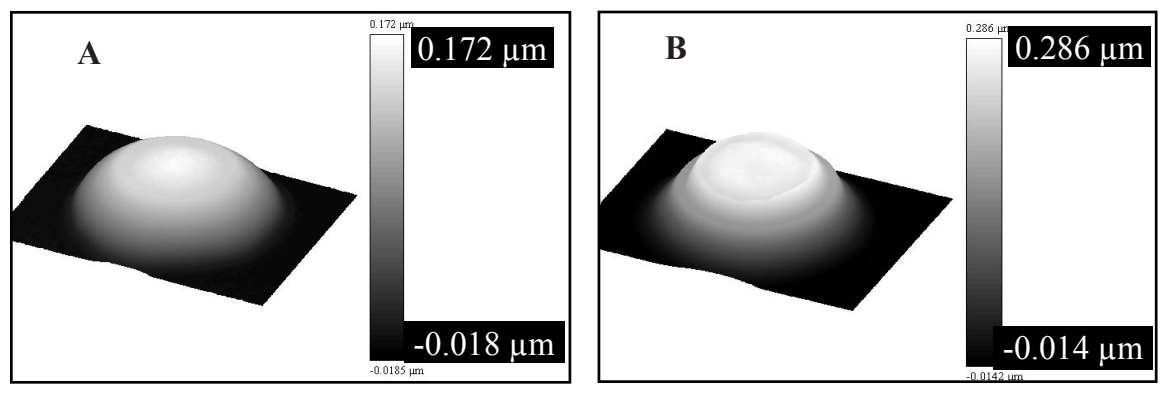

Figure 2.4: 3D interferometric images of inkjet printed $(A)$ single droplet and $(B)$ two droplets (wet-on-wet) on glass.

Even though the exact mechanism of surface roughness formation was not thoroughly investigated, it is hypothesised that the high surface roughness seen in figure 2.3(C) can be attributed to the drying process. As more droplets are stacked, the amount of solvent as well as additives to be removed by heating increases. Since the evaporation-start instant is different for different layers, and since the edges dry at a faster rate than the interior of the printed layers, the drying process occurs in an inhomogeneous manner, 
Chapter 2

resulting in higher roughness. Such observations have also been recorded in $[7,8]$. However, Garnett et al [9] found that the roughness of PEDOT:PSS films increase with increase in substrate temperature. Observations indicated that surface roughness of printed profiles is case-specific, and depends on the type of ink as well as printing and drying parameters.

In addition to the increase in surface roughness with increasing number of layers, it was also observed from the tracks printed using the wet-on-wet approach that the track width increases with the number of layers. This can be clearly seen in figure 2.5 , which depicts the optical microscope images of twice and thrice printed tracks.
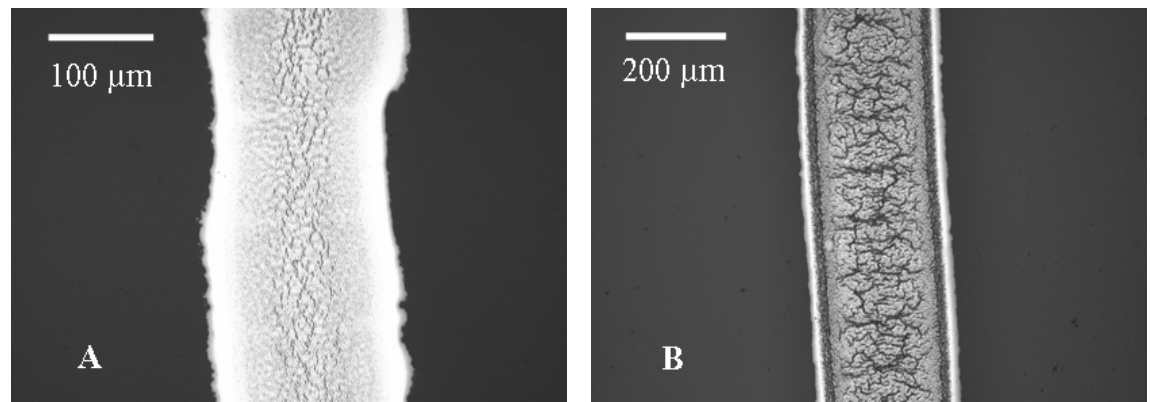

Figure 2.5: Micrographs of inkjet printed tracks (wet-on-wet) on glass, containing (A) two stacked layers and $(B)$ three stacked layers; the irregular surface morphology of $(B)$ is attributed to its high surface roughness. It is obviously broader than the one on the left.

\subsubsection{Wet-on-partial-dry approach}

The wet-on-partial-dry approach yielded better results in terms of the increase in track thickness with the number of layers - the increase was quasi-linear, as shown in figures 2.6. Moreover, there was only a minimal increase in track width. For instance, the increase in the track width of a twice-printed track when compared to a once-printed track was about $15 \%$ of the latter's width. These observations can be attributed to the relative rigidity of the printed layer due to the evaporation of the non-metallic components of the ink. As a result of this, when the next layer was printed, the spreading of the ink was not as pronounced as in the previous case. The printed structures were quite smooth, unlike those printed using the wet-on-wet approach. 
These three criteria, viz. quasi-linear increase in track thickness, minimal increase in track width and low surface roughness values were met satisfactorily by the wet-onpartial-dry approach for up to five stacked layers.
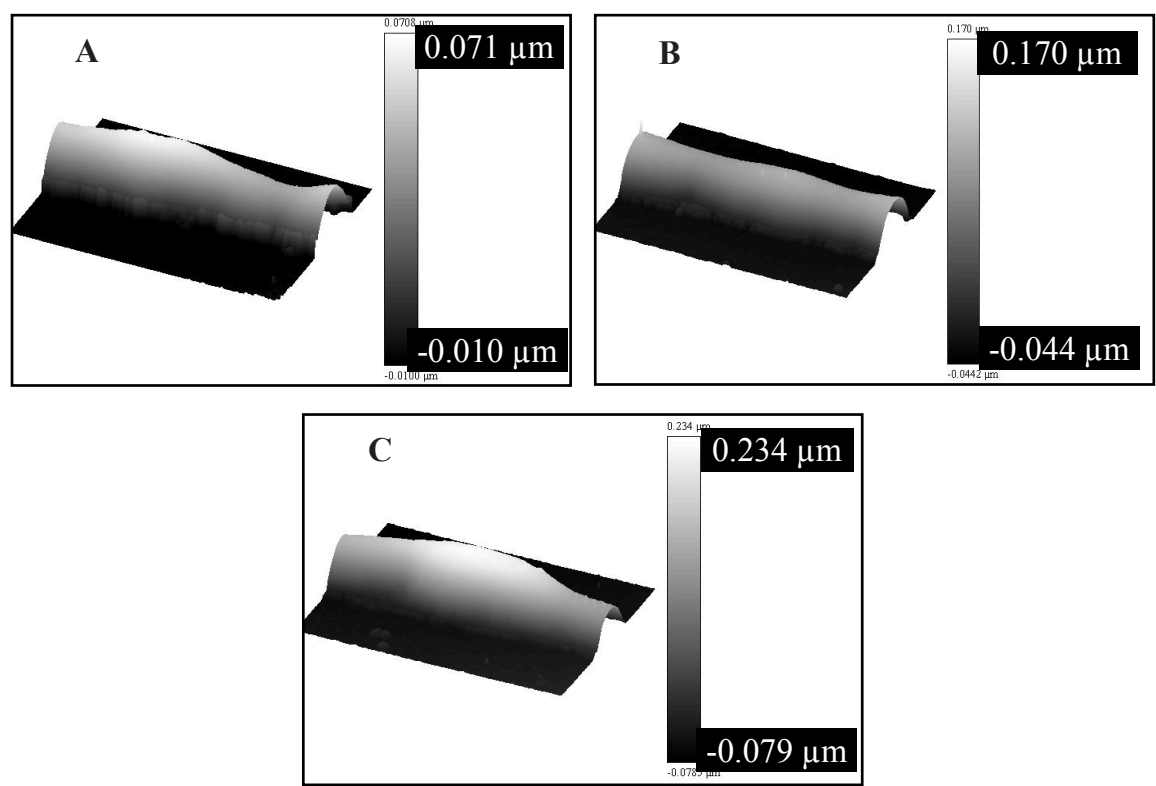

Figure 2.6: 3D interferometric images of inkjet printed tracks (wet-on-partial-dry) on glass, containing $(A)$ one layer, $(B)$ two stacked layers and $(C)$ three stacked layers.
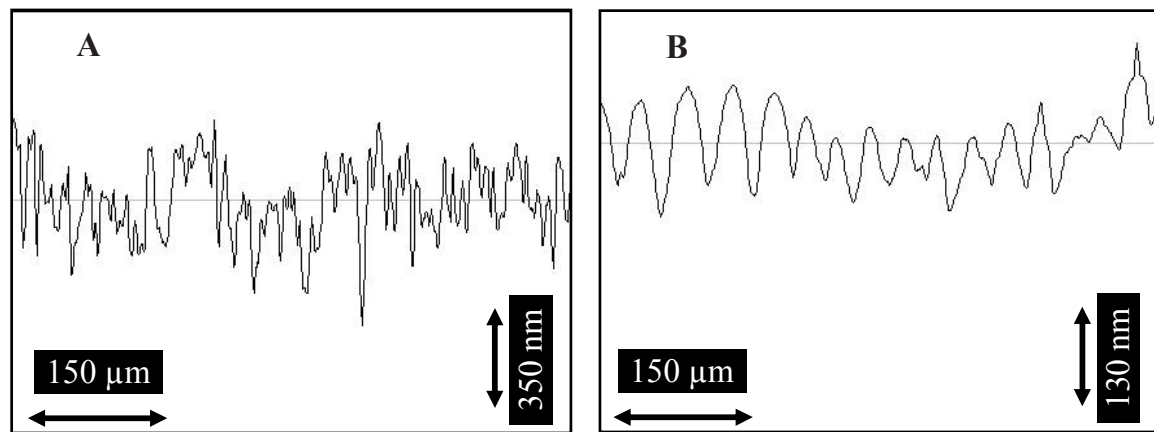

Figure 2.7: Surface roughness comparison of inkjet printed structures with five layers on glass, using $(A)$ wet-on-wet approach and $(B)$ wet-on-partial-dry approach.

Figure 2.7 compares the surface morphology of structures with five stacked layers inkjet printed using the wet-on-wet and wet-on-partial-dry approaches. These structures are 
Chapter 2

not single tracks with a number of stacked layers as discussed above, but they are stacked films which were generated by printing multiple droplets in $\mathrm{X}, \mathrm{Y}$ and $\mathrm{Z}$ directions. It can be clearly seen that while the wet-on-wet approach results in a very rough surface, the wet-on-partial-dry approach results in a much better surface morphology.

\subsection{Conclusions}

The geometrical aspects of inkjet printing of multilayered 3D structures were studied in this chapter. Three different ways of fabricating 3D structures viz. wet-on-wet, wet-ondry and wet-on-partial dry were studied. The droplet pitch is crucial for the build-up of $3 \mathrm{D}$ microstructures, in order to avoid bulging or scalloping of the printed structure. The wet-on-wet approach leads to much wider tracks than intended and hence is not the right approach to print 3D microstructures. Moreover, it also results in higher surface roughness and lower thickness of printed tracks than the corresponding wet-on-partialdry approach.

The wet-on-partial-dry approach was found to be the suitable one for inkjet printing of 3D microstructures. Though only one type of ink was used in this study, this methodology adopted can be applied to other types of inks as well. Under the framework of this research, fabrication of structures requiring inkjet printing of multiple layers one of top of the other i.e. stacking was not pursued further, due to the successful implementation of an alternate approach. This approach involves inkjet printing of a single metallic layer on a substrate and subsequently depositing copper on this layer using electroless plating. This has several advantages that are discussed in chapter 4 .

Nevertheless, further investigations are recommended to ascertain the suitability inkjet printing to fabricate thick structures that need stacking of ten or more inkjet printed layers. The reason being, electroless plating is not suitable for every type of ink used in conjunction with inkjet printing. Moreoever, since it involves dipping of the whole substrate into the plating bath, it does not offer flexibility to the process chain. For example, if the substrate has any pre-fabricated structures/components on which copper 
deposition is not required, they have to be insulated or hermetically sealed prior to plating, to avoid any interaction with the plating solution.

\subsection{References}

[1] J. Perelaer: "Microstructures prepared via inkjet printing and embossing techniques", Ph.D. Thesis - Eindhoven University of Technology, Eindhoven, the Netherlands, 2009.

[2] V. Subramanian, S.K. Volkman and D.R. Redinger: "Printed zinc oxide based electronics: materials, devices, and outlook", Proceedings of the LOPE-C, Frankfurt, Germany, 2009.

[3] U. Geyer, F. Siegel, A. Kreutzer, T. Blaudeck, R. R. Baumann, M. Bohnke, H. Rouault, B. Fillon, L.-O. Hennerdal and M. Loegdlund: "Printing electrode materials for rechargeable lithium thin-film batteries", Proceedings of the LOPE-C, Frankfurt, Germany, 2009.

[4] S.-H. Ko, J. Chung, H. Pan, C.P. Grigoropoulos and D. Poulikakos: "Fabrication of multilayer passive and active electric components on polymer using inkjet printing and low temperature laser processing", Sensors and Actuators A: Physical, Vol. 134, No. 1, p. $161,2007$.

[5] I. Nagy: “Accurate impedance control”, Printed Circuit Design \& Fab, p. 20, November 2009.

[6] V. Sanchez-Romaguera, M.-B. Madec and S.G. Yeates: "Inkjet printing of 3D metal-insulator-metal crossovers", Reactive \& Functional Polymers, Vol. 68, No. 6, p. 1052, 2008.

[7] H.C. Jung, S.-H. Cho, J.W. Joung and Y.-S. Oh: "Studies on inkjet-printed conducting lines for electronic devices", Journal of Electronic Materials, Vol. 36, No. 9, p. 1211, 2007.

[8] T. Kaydanova, M.F.A.M. Van Hest, A. Miedaner, C.J. Curtis, A.L. Alleman, M.S. Dabney, E. Garnett, S. Shaheen and D. Ginley: "Direct write contacts for solar cells", $31^{\text {st }}$ IEEE Photovoltaics Specialists Conference and Exhibition, Florida, USA, January 2005. 
Chapter 2

[9] E. Garnett and D. Ginley: "Electrical and morphological properties of inkjet printed PEDOT/PSS films", U.S. Department of Energy Journal of Undergraduate Research, Vol. 5, p. 24, 2005. 


\section{Inkjet printing of conductive structures on a PCB material}

This chapter describes the issues pertaining to inkjet printing of a silver nanoparticle-based ink on a commercially available PCB material with different surface morphologies. Two variants of the substrate, viz. etched and unetched, were used in order to determine the influence of the surface morphology of the substrate on adhesion as well as accuracy of the printed structures. In the context of this chapter, etching refers to the removal of copper cladding by a chemical etchant. The pull-off adhesion test method was used to quantify adhesion strength. The dependence of the pull-off test results on the local geometry of the test area is illustrated with the aid of scanning electron microscope (SEM) images and interferometric studies. The main conclusion of this chapter concerns the nature of substrate surface morphology that is suitable for inkjet printing, and the conflicting requirements posed by adhesion as well as printing accuracy on surface morphology.

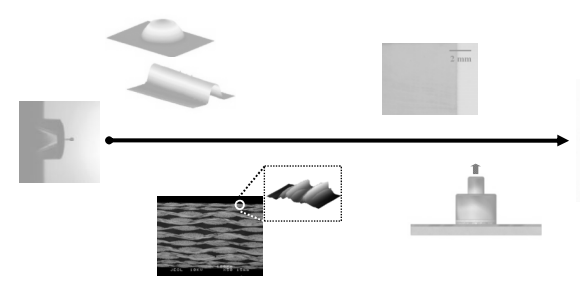


Chapter 3

\subsection{Introduction}

The potential of inkjet printing as a method to deposit functional inks on various substrate materials has been widely published [1-6]. However, the substrates discussed in most contemporary scientific publications are either idealised materials like glass or relatively smooth, flexible films like polyimide and polyester. Rigid, fibre-reinforced PCB materials that are widely used in the electronics industry have seldom been discussed in the context of inkjet printing.

Figure 3.1 shows the cross-section of a rigid substrate. Such a substrate is usually manufactured by pressing thin laminates, known as prepregs, together and allowing the thermoset matrix material to cure. Each prepreg has fibre reinforcement. Special electronic grade glass fibre, known as E-glass, is usually used for this purpose. Substrates of this type may have filler materials in addition to the matrix material and the fibre reinforcement. The fillers as well as the fibre reinforcement lend the desired mechanical, electrical and chemical properties to the substrate, and their composition depends on the nature of application of the end product. In general, the surface morphology and the surface mechanisms of the substrate are predominantly governed by the matrix material.

The process of manufacturing of a rigid substrate from prepegs is shown in figure 3.2. It is common practice to press copper foils together with the prepegs, so that the copper can be etched off at a later stage to create electronic circuit interconnections. These copper foils are, by and large, produced by an electrodeposition process, even though rolled copper foils are used as well. A copper foil produced by electrodeposition is smooth on one side that is in contact with the polished stainless steel drum that acts as the cathode; the other side is microscopically rough as a result of columnar copper grain growth during electrodeposition [7]. When the rougher side of a copper foil is pressed together with the prepregs at temperatures exceeding the glass transition temperature $\left(\mathrm{T}_{\mathrm{g}}\right)$ of the matrix material of the prepreg, the matrix material deforms around the roughness peaks and valleys of the copper foil. This provides mechanical interlocking (anchoring) upon cooling. 


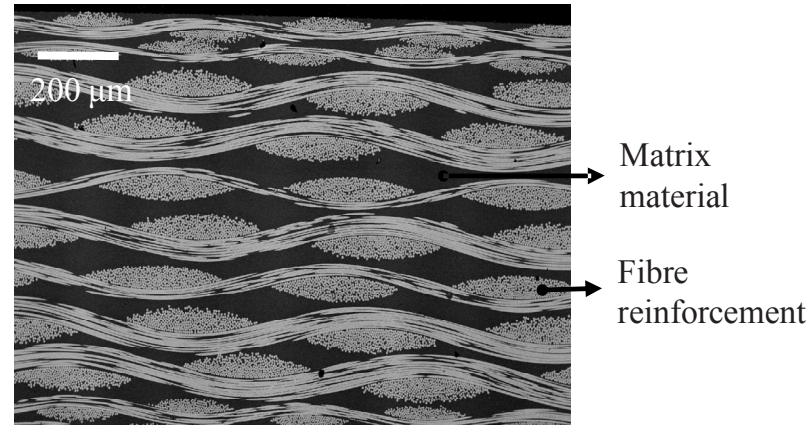

Figure 3.1: Cross-section of a fibre-reinforced PCB material.

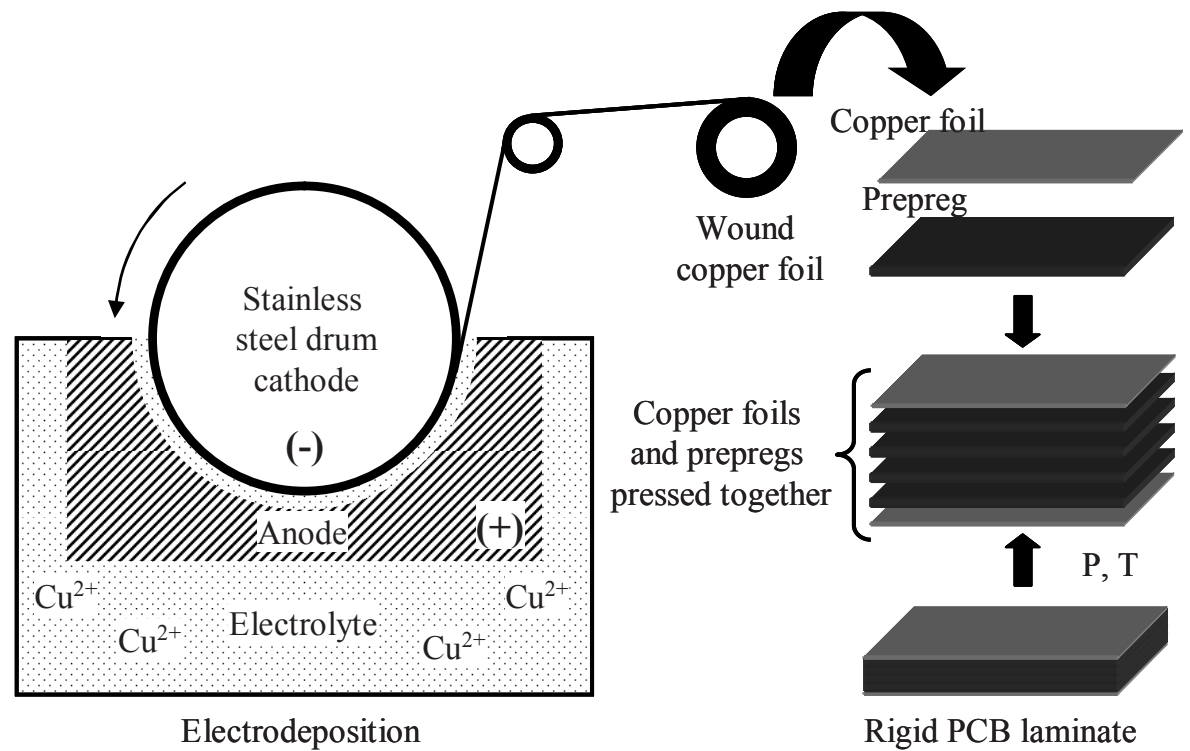

Figure 3.2: Manufacture of rigid PCB laminate.

In this chapter, significant observations and challenges encountered during inkjet printing on reinforced PCB substrates, manufactured using the procedure just described, are reported. 
Chapter 3

\subsection{Experimental details}

\subsubsection{Materials and methods}

A commercially available E-glass reinforced epoxy material (FR4 from Park Electrochemical Corp., USA) was used as the substrate. The procured FR4 substrate, with a nominal thickness of $1.6 \mathrm{~mm}$, had a copper cladding. Initial printing trials were done after etching off this cladding using the conventional chemical etching process widely used in the PCB industry. A plasma cleaning step was subsequently carried out to remove the contaminants. Another type of FR4 substrate was used in the later stages of this study. This substrate, also from the same supplier, had no copper cladding, and was relatively flexible with a thickness of $0.1 \mathrm{~mm}$. Since it had no copper cladding, it was not subjected to etching. When compared to the substrate used for the initial printing trials, this substrate comprised a smaller number of plies. Conductive structures were printed using Harima silver nanopaste, containing approximately $60 \%$ silver nanoparticles by weight.

Two test methods were used to characterise the adhesion between the printed structures and the substrate: the Scotch-tape test and the pull-off test. The Scotch-tape test is a method for evaluating the adhesion of a coating to the substrate using a pressuresensitive tape. However, it is not possible to quantify adhesive strength using this test method. It was used to identify which of the two substrate types, viz. rigid substrate with an initial copper cladding and flexible substrate with no cladding, was best suited for inkjet printing and subsequent characterisation. Since the Scotch-tape is a pressuresensitive tape, it was applied on each specimen in a fairly controlled manner with the aid of a roller, to make the test specimen preparation process repeatable.

The pull-off test is an ISO-standardised method widely used in the paint and varnish industry to quantify adhesion [8]. A schematic of this test method is shown in figure 3.3. In this method, an aluminium dolly (stud) is glued to the specimen whose adhesion or cohesion is to be tested, and a tensile load is applied until failure. For this study, aluminium dollies with a diameter of $10 \mathrm{~mm}$ were used and Araldite 2011, a twocomponent epoxy-based adhesive, was used to glue them to the test specimens. PosiTest AT, a portable pull-off tester (from DeFelsko Corp., USA), was used for adhesion 
characterisation. This tester exerts tensile loads generated by a hand-operated hydraulic pump. SEM was used to characterise the surface morphology of the substrates. The operating voltage of the SEM was $20 \mathrm{kV}$. Interferometric analyses were also performed to determine the surface roughness of the substrates. Fracture surfaces resulting from pull-off testing were viewed and analysed under an optical microscope.

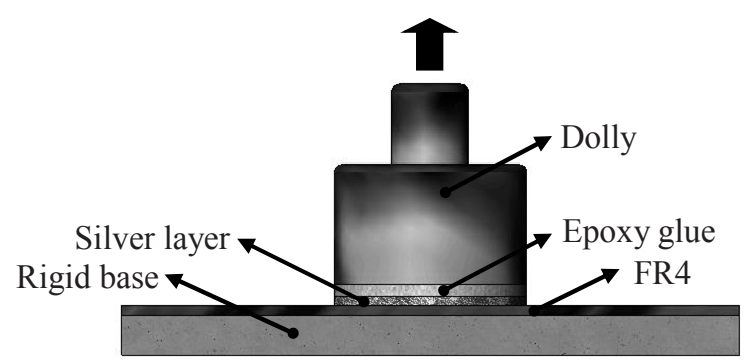

Figure 3.3: Schematic representation of the pull-off test method; the block arrow indicates the pulling direction.

\subsubsection{Specimen preparation and testing}

A set of identical silver structures was inkjet printed for Scotch-tape testing and pull-off testing. Prior to printing, all substrates were cleaned and dried in a convection oven. The substrate holder was pre-heated to $55^{\circ} \mathrm{C}$ and maintained at that temperature during printing, to accelerate the evaporation of the solvent i.e. tetradecane. The bipolar waveform shown in figure 3.4 [9] was used to actuate the nozzle. The wave parameters (voltage in terms of volts and time in terms of $\mu$ ) are also shown in this figure. The dimensions of the printed structures were $10 \mathrm{~mm} \times 10 \mathrm{~mm}$ (length $\times$ width), and their nominal thickness was $0.6 \mu \mathrm{m}$. Figure 3.5 shows an inkjet printed silver structure similar to those subjected to adhesion tests. Even though the dollies used in pull-off testing had a circular base with a diameter of $10 \mathrm{~mm}$, the structures were not printed to exactly match it, to avoid irregularities along the circumference of a circular profile, as shown in figure 3.6. The dotted line in the figure represents the desired edge. This deviation from the desired edge, termed as the 'staircase effect' [10], was caused due to the lack of combined X-Y movement of the machine stages while printing. 
Chapter 3

The printed structures were subsequently sintered at $210^{\circ} \mathrm{C}$ in a convection oven for 60 minutes, as specified by the ink supplier, to impart continuity. After sintering, Scotchtape tests were performed on some of the test specimens. Upon analysing the results of the tests, it was decided to perform the quantifying pull-off tests only on the structures printed on the unclad, flexible FR4 substrate. The reasons behind this are explained in the next section.

Four test specimens, henceforth referred as FR4-Ag 1 to FR4-Ag 4, were subjected to pull-off tests. They had to be glued to a rigid base prior to testing. This is due to the fact that during pull-off testing of flexible substrates, high tensile stress peaks tend to concentrate near the edges of the dolly. The bending of the substrate during testing is the cause of the uneven stress distribution, which decreases the force needed to induce fracture, as confirmed by [11]. In the next step, the dollies were glued to the test specimens. The excess glue present between the dolly and the test specimen was extruded from the test area before curing, by exerting a compressive load acting vertically on the dolly, after which it was wiped off with a soft cloth. In spite of wiping, a thin layer of glue still remained around the test area. Upon curing of the glue, a cutting tool was used to isolate the test area, to prevent the surrounding glue layer from influencing the test results, as the local geometry dominates the local stress distribution and hence the pull-off load. All the pull-off tests were performed at a constant rate of load application (0.5 MPa. $\left.\mathrm{s}^{-1}\right)$, to ensure consistency.

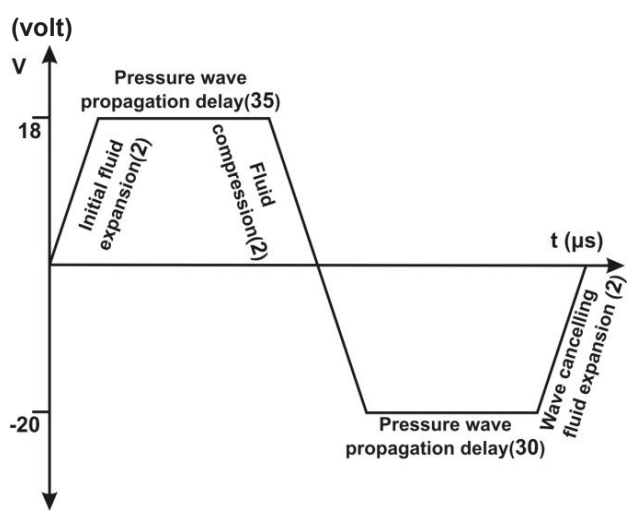

Figure 3.4: The bipolar waveform used for actuating the piezoelectric drop-on-demand nozzle; the wave parameter values are also shown. 


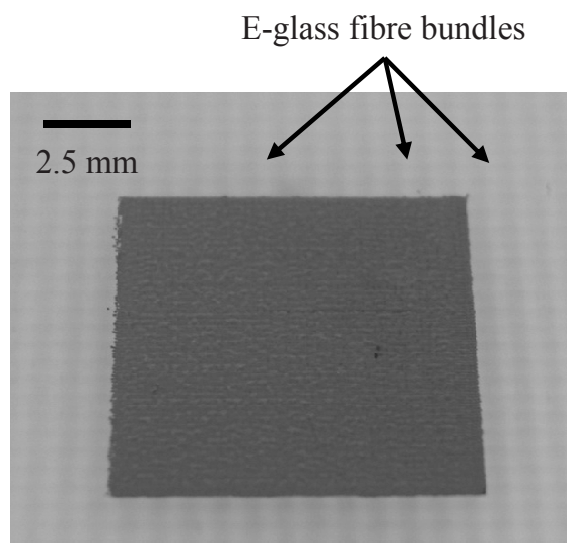

Figure 3.5: Inkjet printed silver structure (a square) with a nominal thickness of $0.6 \mu \mathrm{m}$, on FR4. Due to the translucent nature of the substrate, the E-glass fibre bundles very close to the surface of the substrate are visible in this figure.

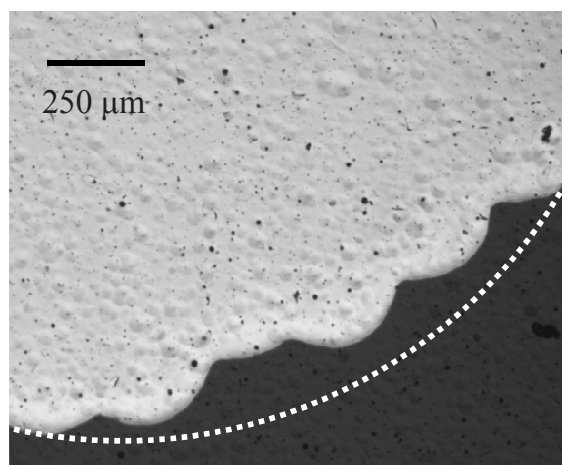

Figure 3.6: Edge irregularity of a printed circular profile; the dotted line represents the desired edge.

\subsection{Results and discussion}

\subsubsection{Etched substrate}

After printing and sintering the structures on the etched substrate, it was found that characterising them was extremely difficult, as the process of etching left behind a dense and uneven distribution of surface pores. This surface porosity, created due to the roughness of the etched copper cladding, resulted in a very high proportion of the printed structures to lie inside the pores. As mentioned before, during the substrate 
Chapter 3

manufacturing process at the supplier's end, the cladding is deliberately kept rough on one side, so that the matrix material i.e. epoxy deforms around these roughness profiles during the pressing stage and mechanical interlocking is promoted. The diameters of the resulting surface pores were found to be in the order of $2 \mu \mathrm{m}$ or more as shown in the SEM images in figure 3.7, and their depths, as high as $10 \mu \mathrm{m}$. It can also be seen from this figure that most of these pores are so close to each other, that they should be considered as closely-packed clusters of pores instead of individual entities. This is true not just for FR4, but also for other PCB materials such as RO4003, a glass fibre reinforced hydrocarbon/ceramic material (from Rogers Corp., USA) that is typically used in high frequency circuits.
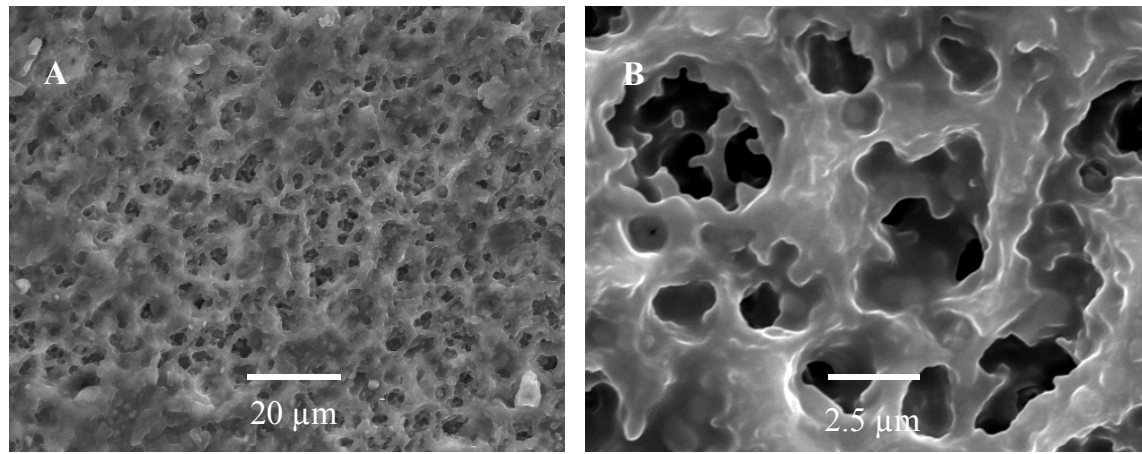

Figure 3.7 $(A \& B)$ : SEM images of the reinforced epoxy resin substrate at different magnifications.

\subsubsection{Scotch-tape testing}

The porous surface morphology of the etched substrate also led to complications during adhesion characterisation, exemplified by the Scotch-tape tests done on the printed structures. Figure 3.8 compares the results of the Scotch-tape test done on the substrate under discussion, with the result of the test done on the unclad (and hence unetched) substrate. In the case of the former substrate, a substantial portion of the test geometry remained within the substrate while, in the case of the latter substrate, which will be discussed later in detail, almost the entire test structure was peeled off with the tape. 


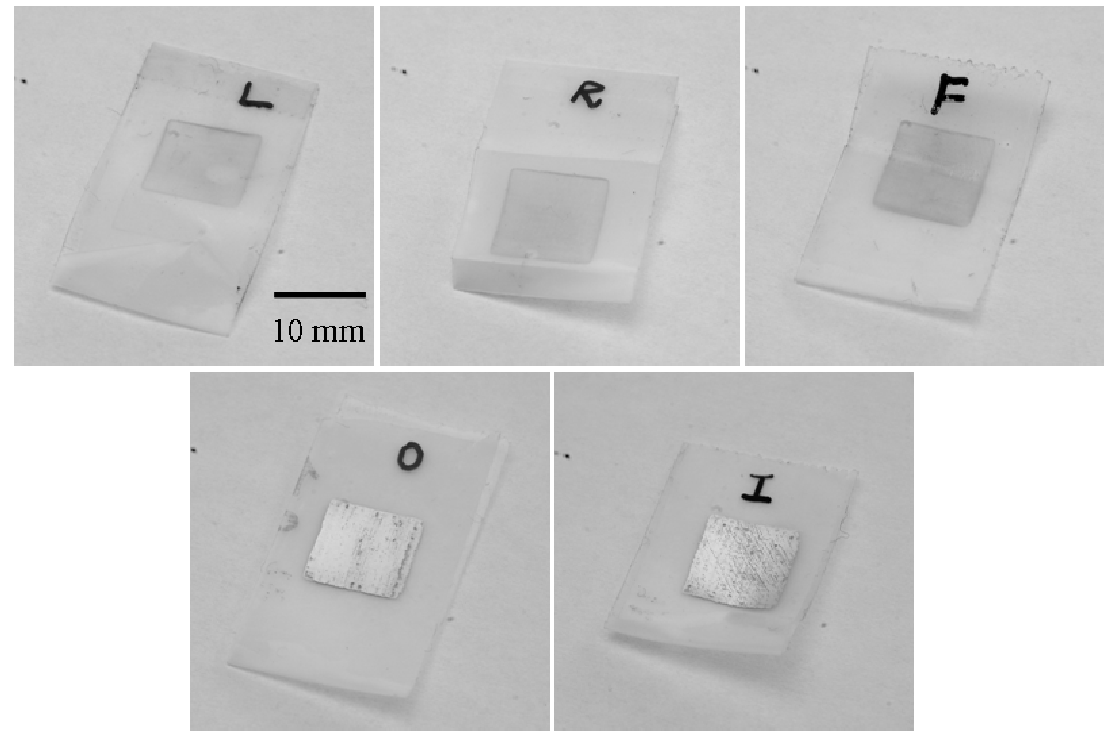

Figure 3.8: Scotch-tapes using which silver structures were peeled off from etched FR4 substrates (top) and unetched FR4 substrates (bottom); multiple specimens shown here to demonstrate consistency of the test procedure. The letterings on the specimens are not relevant for this discussion.

From these results, it can be inferred that the application of inkjet printing as a re-work tool to repair or replace components like resistors or conductive structures on an existing PCB, as proposed in [12], is not straightforward. Printing on existing PCBs that have undergone etching would result in a highly uncontrolled deposition, as the lowviscous, picolitre-sized ink droplets will spread into the gaping surface pores, resulting in printed structures having discontinuities as well as varying thicknesses and widths. In the context of the current research, such structures may result in a deviation from the desired RF performance level.

\subsubsection{Substrate heating}

During the course of this research, inkjet printing was done on porous substrates that were heated up to and maintained at $100{ }^{\circ} \mathrm{C}$. It was observed that by doing so, the spreading of the ink on the substrate was minimised not only in the plane of printing, but also through the substrate thickness. Quite logically, the solvent present in the ink evaporated much quicker on a heated substrate due to flash-evaporation, than an 
Chapter 3

unheated one. However, heating the substrate cannot completely prevent the flow of the ink into the pores. In addition to that, at high substrate temperatures, the viscosity of the ink in the nozzle decreases due to its proximity to the substrate, as discussed in [13]. Even if the substrate is maintained at a constant temperature, the temperature of the nozzle and hence the ink increases until equilibrium is reached, as shown in figure 3.9.

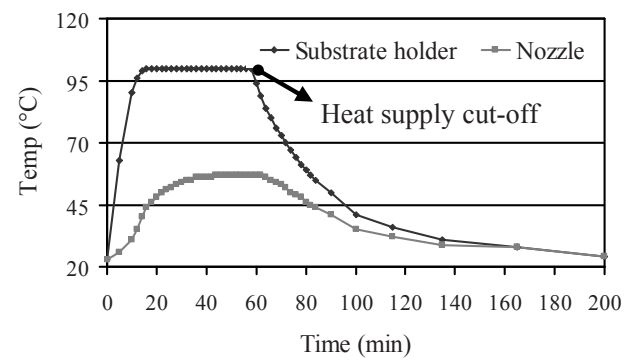

Figure 3.9: Time evolution of the substrate holder and nozzle temperatures due to the heating of the substrate.

For a given parameter set and pressure wave at the nozzle, the lower the viscosity, the higher the velocity and the volume of fluid pushed forward, leading to formation of long tails behind the droplet [14]. This will result in larger droplets than intended. Even though this problem was circumvented in the study presented in chapter 2, it is obviously uneconomical and unsuitable for series production. Hence, this approach has its limitations. For this reason, it was concluded that in order to integrate inkjet printing into the PCB manufacturing chain as a complementary process or as a re-work process, substrates with favourable morphology are needed. In other words, there should be a compromise between the roughness of the copper cladding and its desired adhesion to the substrate, so that inkjet printing could be done on etched substrates as well.

\subsubsection{Unetched substrate}

If inkjet printing is thought of as a stand-alone fabrication process for the PCB industry i.e. if the intention is to fabricate complete circuits using only inkjet printing (or, for that matter, any other additive process), procuring substrates with copper cladding and then etching the copper away does not make sense. Instead, such a fabrication process would involve printing on unclad substrates, thereby eliminating additional process steps and 
material wastage. Hence, it was decided to inkjet print on unclad substrates. The results in this case were, as expected, better than those obtained using the other substrate type, as depicted by the Scotch-tape tests discussed already.

\subsubsection{Pull-off testing}

The results of the pull-off tests are graphically represented in figure 3.10 as the maximum force divided by the loaded area. It is clear from the graph that the adhesion values of the test specimens FR4-Ag 1 to FR4-Ag 4 are low in comparison with the average value of tests done on the conventional PCB (FR4-copper), obtained from the literature [15]. However, the reference FR4-copper interface was strong due to the same reason discussed in the beginning of this section: adhesion of copper to epoxy was enhanced by the mechanical interlocking due to the surface roughness of copper.

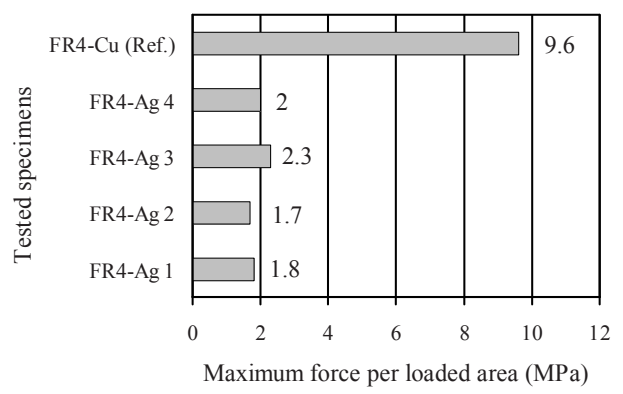

Figure 3.10: Results of the pull-off tests on inkjet printed silver structures on the unclad FR4 substrate; reference value from [15].

The fracture mode of the test specimens was basically cohesive-adhesive, i.e. in some areas of the test specimens the failure occurred within the silver structure and in other areas, it occurred at the substrate-silver interface. This is evident from figure 3.11. This figure also supplies additional information pertaining to the substrate morphology. The crimping of the fibres of the bidirectional fibre-reinforcement is visible on the surface of the substrate. The surface roughness created as a result of this crimping is quite pronounced, as shown by the interferometer image depicted in figure 3.12. In this figure, the peaks represent the individual fibre filaments of a bundle that are projecting outwards from the surface of the substrate. 
Chapter 3

The mechanism of fracture of the test specimens under study was heavily influenced and complicated by the crimping of the fibres. When a droplet from the nozzle of the printer falls on a spot where the fibre bundles project from the surface of the substrate, it will initially be in contact with two different materials - E-glass and epoxy simultaneously. These materials have different surface energies, resulting in varying contact conditions. The ink droplet will then flow into the channels between two adjoining fibre filaments. It can be clearly seen from the SEM image of an inkjet printed substrate, depicted in figure 3.13, that silver is concentrated at the edges of the filaments. It is also evident from this SEM image that the thickness of the silver layer trapped between two adjacent fibre filaments is greater than the layer thickness beyond the 'zone of crimping'. Thus, in the context of pull-off testing, the crimping of the fibres leads to:

1. Variation in thickness of the silver structure and the glue layer.

2. Direct contact between the glue and the projecting fibre bundles as a result of discontinuities in the silver structure.

3. Creation of new edges, resulting in uneven stress distribution in the test area.

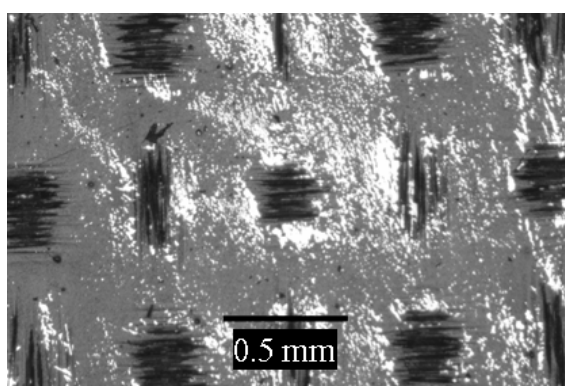

Figure 3.11: Optical microscope image of an inkjet printed substrate after pull-off testing, showing the cohesive-adhesive fracture of the silver structure. The bright areas are the remnants of silver. 


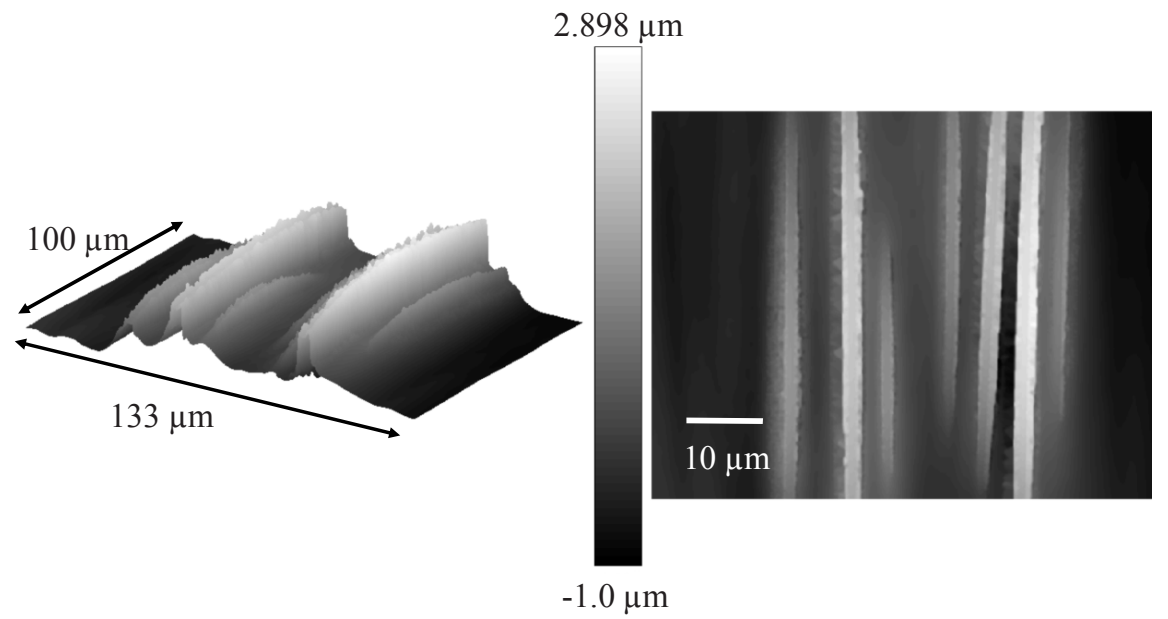

Figure 3.12: The surface roughness $\left(\mathrm{S}_{\mathrm{a}}\right)$ of the unclad substrate, in $3 \mathrm{D}$ view (left) and $2 \mathrm{D}$ view (right) using interferometry; the peaks and the valleys represent the crimping of the fibre reinforcement.

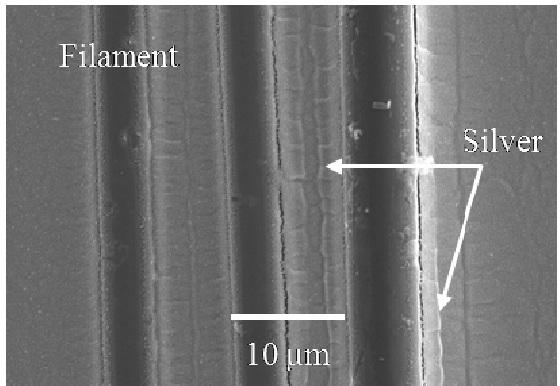

Figure 3.13: Accumulation of printed silver at the edges of the fibres as well as between two fibres, as viewed from the top.

\subsection{Conclusions}

Microscopic studies showed that the printed structures on the unclad FR4 were rather continuous and uniformly thick, except the places where the fibre bundles protrude from the surface of the substrate. From the outcomes of the printing experiments and adhesion tests, it can be concluded that though the unclad substrate is quite suitable for inkjet printing, further development and optimisation of the surface morphology of fibre-reinforced substrates are needed, to cater to the demands of inkjet printed 
Chapter 3

electronics. The accuracy of droplet positioning is bound to be affected as a result of the undulations caused by crimping of the fibre bundles. In addition, the surface roughness created by the protruding fibres poses problems during adhesion characterisation. On the other hand, these fibre intersections could be considered as 'adhesion promoters' that act as anchoring locations for the printed structures, resulting in better mechanical interlocking in comparison with a smooth substrate.

Optimal substrate morphology should lend itself to accurate printing as demanded by RF applications, in addition to being favourable to better adhesion of the printed ink to the substrate. As mechanical interlocking promoted by surface roughness is a dominant factor that determines adhesion, these rather contradictory requirements need a more case-specific approach to find out the suitable substrate surface morphology for applications within the electronics industry.

The relatively high sintering temperature $\left(210^{\circ} \mathrm{C}\right)$ of the nanoparticle-based ink is another area that needs attention, especially for electronics applications. The glass transition temperature $\left(\mathrm{T}_{\mathrm{g}}\right)$ of most of the materials used as PCB substrates, including the one used in this research, is lower than $200^{\circ} \mathrm{C}$. By heating the material beyond its $\mathrm{T}_{\mathrm{g}}$ value and subsequently cooling it induces residual stresses that could have a negative effect on the printed structures as well as the interface between the structures and the substrate. Inks with a lower sintering temperature $\left(150^{\circ} \mathrm{C}\right.$ and below) have already been developed, along with alternate sintering methods like microwave sintering [16], UVenhanced annealing [17] and laser sintering [18]. These developments will go a long way in aiding the commercialisation of inkjet printed electronics on all kinds of substrate materials.

\subsection{References}

[1] Y. Liu, T. Cui and K. Varahramyan: “All-polymer capacitor fabricated with inkjet printing technique", Solid-State Electronics, Vol. 47, No. 9, p. 1543, 2003.

[2] D. Redinger, S. Molesa, S. Yin, R. Farschi and V. Subramanian: "An ink-jetdeposited passive component process for RFID”, IEEE Transactions on Electron Devices, Vol. 51, No. 12, p. 1978, 2004. 
[3] E. Tekin, B-J. de Gans and U.S. Schubert: "Ink-jet printing of polymers - from single dots to thin film libraries", Journal of Materials Chemistry, Vol. 14, No. 17, p. 2627, 2004.

[4] Y. Yoshioka, P. D. Calvert and G. E. Jabbour: "Simple modification of sheet resistivity of conducting polymeric anodes via combinatorial ink-jet printing techniques", Macromolecular Rapid Communications, Vol. 26, No. 4, p. 238, 2005.

[5] Y. Liu and T. Cui: "Polymer-based rectifying diodes on a glass substrate fabricated by ink-jet printing”, Macromolecular Rapid Communications, Vol. 26, No. 4, p. 289, 2005.

[6] T.H.J. van Osch, J. Perelaer, A.W.M. de Laat and U.S. Schubert: "Inkjet printing of narrow conductive tracks on untreated polymeric substrates", Advanced Materials, Vol. 20, No. 2, p. 343, 2008.

[7] M.W. Jawitz and M.J. Jawitz: "Materials for rigid and flexible printed wiring boards", Taylor \& Francis Group, p. 66, 2007.

[8] "Paints and varnishes - Pull-off test for adhesion", ISO 4624:2002, 2002.

[9] D.B. Wallace, V. Shah, D.J. Hayes and M.E. Grove: "Photo-realistic ink-jet printing through dynamic spot size control”, Journal of Imaging Science \& Technology, Vol. 40, No. 5, p. 390, 1996.

[10] R.L. Hope, R.N. Noth and P.A. Jacobs: "Adaptive slicing with sloping layer surfaces”, Rapid Prototyping Journal, Vol. 3(3), p. 89, 1997.

[11] M.P.K. Turunen, P. Marjamaki, M. Paajanen, J. Lahtinen and J. K. Kivilahti: "Pulloff test in the assessment of adhesion at printed wiring board metallisation/epoxy interface”, Microelectronics Reliability, Vol. 44, No. 6, p. 993, 2004.

[12] J.J. Howarth, C. Edwards and K. Vanheusden: "Replacement of passive electrical components", U.S. Patent Application Publication No. US 2006/0176350 A1, 10 Aug. 2006.

[13] A. Sridhar, D.J. van Dijk and R. Akkerman: "Inkjet printing of functional inks on PCB materials", Proceedings of the EIPC Winter Conference, Rome, Italy, 2008.

[14] B.J. de Gans, P.C. Duineveld and U.S. Schubert: "Inkjet printing of polymers: state of the art and future developments", Advanced Materials, Vol. 16, No. 3, p. 203, 2004.

[15] J. Ge, R. Tuominen and J.K. Kivilahti: “Adhesion of electrolessly-deposited copper to photosensitive epoxy", Journal of Adhesion Science and Technology, Vol. 15, No. 10, p. $1133,2001$. 
Chapter 3

[16] J. Perelaer: "Microstructures prepared via inkjet printing and embossing techniques", Ph.D. Thesis - Eindhoven University of Technology, Eindhoven, the Netherlands, 2009.

[17] M. Mueller, M. Knothe, J. Wend, S.F. Jahn, T. Blaudeck and R.R. Baumann: "UVenhanced thermal annealing of inkjet-printed silver structures", Proceedings of Printing Future Days, Chemnitz, Germany, 2009.

[18] S.H. Ko, H. Pan, C.P. Grigoropoulos, C.K. Luscombe, J.M.J. Frechet and D. Poulikakos: "All-inkjet-printed flexible electronics fabrication on a polymer substrate by low-temperature high-resolution selective laser sintering of metal nanoparticles", Nanotechnology, Vol. 18, No. 34, DOI: 10.1088/0957-4484/18/34/345202, 2007. 


\section{Plasma pre-treatment for inkjet printing- and electroless plating-based fabrication of conductive circuit structures}

A method to fabricate conductive (RF-) electronic circuit structures is described. This method involves inkjet printing of a metallic ink on a polymeric substrate to create the seed layer for a subsequent electroless copper plating process. This approach has several advantages, and they are discussed in detail in this chapter with the aid of structures fabricated on RO4003 substrate material.

Prior to inkjet printing, surface treatment of the substrate was necessary to improve the adhesion between the substrate and the seed layer. For this purpose, a $\mathrm{CF}_{4} / \mathrm{O}_{2}$ plasma etching process was selected. In order to identify the optimum surface characteristics of the substrate, a statistical design of experiments (DoE) based on a central composite rotatable design (CCRD) was used. The CCRD enables a systematic variation of process parameters and the exploration of process characteristics. The substrates subjected to plasma treatment based on the designed parameter sets were analysed in terms of surface energy, surface roughness and adhesion. From the outcomes of these analyses, substrates with favourable surface characteristics for inkjet printing-electroless plating were identified.

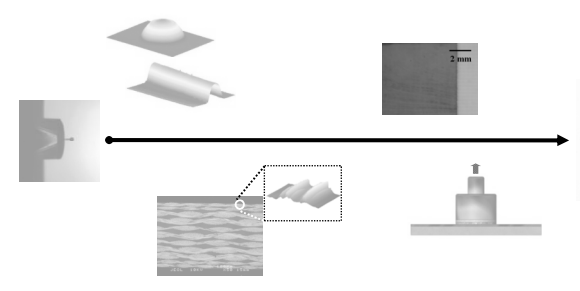


Chapter 4

\subsection{Introduction}

In chapter 2, two approaches to inkjet print 3D structures were discussed: wet-on-wet and wet-on-dry. It was shown that a variant of the wet-on-dry approach, namely the wet-on-partial-dry, is suitable for fabricating 3D structures. It was also mentioned in that chapter that during this research, a totally different approach was followed to buildup the thickness of conductive circuit structures. In this approach, a single silver seed layer is inkjet printed on a polymeric substrate, after which copper is deposited on the silver layer using an electroless plating process. The silver seed layer acts as a catalyst for copper deposition, thus making the electroless plating process highly selective. The mechanism of electroless copper plating was discussed in chapter 1 .

There are several advantages of 3D build-up of conductive circuit structures by inkjet printing-electroless plating process combination, when compared to purely inkjet-based fabrication:

- The conductivity of inkjet printed materials is much lower when compared to bulk metals [1,2], due to pores, cracks, surfactants and other impurities/imperfections. The conductivity values of silver structures inkjet printed during the course of this research did not exceed $16 \%$ of the conductivity of bulk silver. Similar conductivity values for silver inkjet inks have been reported by Perelaer [3] and Valeton [4]. Hence, metallization that ensures conductivity close to bulk copper (which is roughly the same as that of silver) is necessary for electronics applications. The high electrical conductivity of electroless plated copper on silver seed layers has been discussed by Kao et al [5].

- Soldering is an integral process in electronics fabrication. Experiments were conducted to determine whether it is possible to solder on inkjet printed structures. The results showed that even at a solder temperature of $200^{\circ} \mathrm{C}$, the molten solder damaged the printed structure, as shown in figure 4.1(A). The same phenomenon was observed on thicker silver structures (about $100 \mu \mathrm{m}$ thickness) as well. The molten solder seemed to dissolve inkjet printed silver. According to Van Bellegem et al [6] soldering on silver results in the formation of an $\mathrm{Ag}_{3} \mathrm{Sn}$ layer due to the dissolution of silver in tin. The difficulty in 
soldering on inkjet printed structures has also been reported by Mantysalo et al [2]. On the other hand, the structures fabricated using inkjet printing-electroless plating combination were found to be favourable to soldering, as shown in Figure 4.1(B). Soldering temperatures of up to $300^{\circ} \mathrm{C}$ were applied without any visually observable change in these structures.
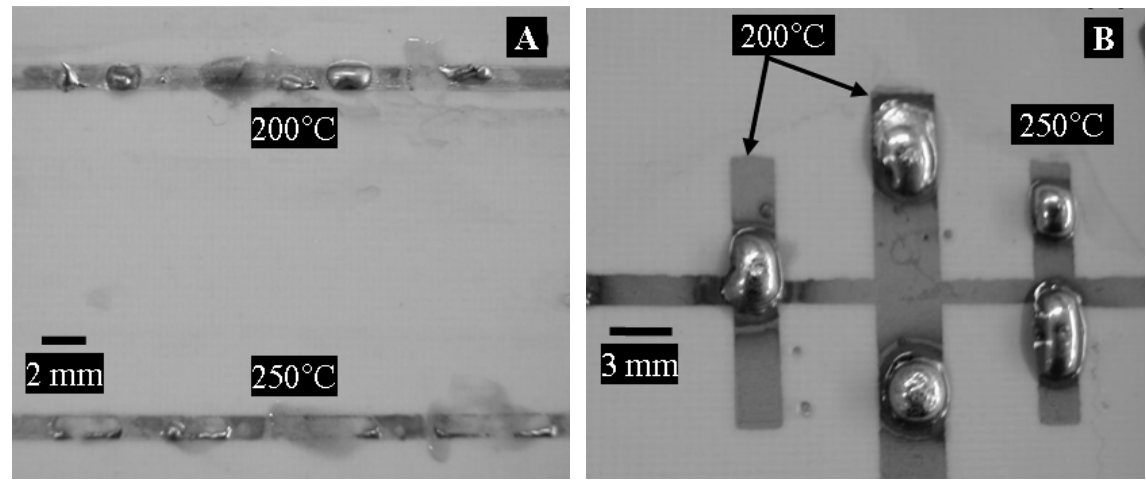

Figure 4.1: (A) Inkjet printed silver structures on a RO4003 substrate dissolved due to soldering; (B) Successful deposition of solder on inkjet printed-electroless plated structures on a RO4003 substrate. The soldering temperatures are shown in the figure.

- A single inkjet printed layer is typically about $1 \mu \mathrm{m}$ or less in thickness. If structures with greater thicknesses are desired, multiple layers should be printed and subsequently sintered. There is a problem here: the organic ligands encapsulating the nanoparticles present in a typical inkjet ink have to be removed during the sintering step. There should be little or no residual ligands left after sintering for the printed structure to be sufficiently conductive. However, if multiple layers are printed before sintering, the sheer thickness of the final layered structure could prevent the complete removal of the organic component of the ink from the inner layers. This results in contamination within the printed structure, which is undesirable [7]. Even in the case of the wet-onpartial-dry approach, where the substrate is heated during the printing process, the complete removal of ligands may not occur due to the relatively low process temperature as well as the short duration of heating.

- Irregularities like pores and micro-cracks that occur on inkjet printed structures as a result of sintering could be covered up by the plated copper. Since the 
Chapter 4

specimen to be plated is completely immersed in the plating bath, the plating solution penetrates cracks and pores. This initiates copper growth in those locations, leading to a continuous structure. The continuity of the inkjet printedelectroless plated structures is of utmost importance for RF performance as well as mechanical reliability.

Prior to the fabrication of conductive circuit structures using the chosen method, the polymeric substrate was subjected to plasma etching using a $\mathrm{CF}_{4} / \mathrm{O}_{2}$ gas combination. The effect of plasma etching on the substrate is two-fold: (1) it activates the surface of the substrate, thereby making it favourable to chemical bond formation with the deposited silver ink, and (2) it roughens the substrate surface, thereby creating anchoring locations for the deposited ink. This enhances the mechanical interlocking between the substrate and the ink, upon the latter's drying. Both these factors contribute to improvement in interfacial adhesion. The importance of surface roughness and chemical bonding for good adhesion in metal-polymer systems has been widely published [8-15]. The role of plasma etching in enhancing interfacial adhesion in metalpolymer systems has also been given sufficient attention [15-18]. Besides the abovementioned effects of plasma etching, one of the main reasons for choosing it as a tool for surface modification is its widespread commercial application in the PCB industry. It is a very versatile process applicable for a wide variety of PCB materials.

During this study, the plasma etching process was used to impart varying degrees of roughness and surface energy to the substrate material, by varying the etch parameters systematically. For this purpose, an experimental design based on a second-order CCRD was used. Finally, the optimal surface characteristics of the substrate were identified based surface energy calculations, surface roughness measurements and adhesion tests using the Scotch tape test method. The main criterion for identifying the optimal surface characteristics of the substrate was improved adhesion compared to an untreated substrate, without affecting the printing accuracy. 


\subsection{Experiments}

\subsubsection{Materials}

The materials and equipments used for this study are listed in table 4.1:

Table 4.1: Details of raw materials and equipments relevant to this chapter.

\begin{tabular}{|l|l|l|}
\hline Raw material/equipment & Type/Name & Supplier \\
\hline Substrate & RO4003 & Rogers Corporation, USA \\
Plasma etching setup & TePla 3067-E & $\begin{array}{l}\text { Technics Plasma GmbH, } \\
\text { Germany }\end{array}$ \\
Inkjet ink & Organic silver complex & InkTec Company Limited, Korea \\
Electroless copper plating system & Envision-2130 & Enthone Inc., USA \\
Cleaning prior to plating & Neutraclean & Shipley Company, USA \\
Contact angle measurement & OCA (optical system) & Dataphysics Instruments GmbH, \\
Surface roughness measurement & DEKTAK surface & Veeco Instruments Inc., USA \\
Electron microscope & profiler & JEOL Limited, Japan \\
\hline
\end{tabular}

\subsubsection{Initial inkjet printing-electroless plating trials without plasma treatment}

To study the possibility of electroless plating on inkjet printed silver seed layers, test structures like the ones shown in figure 4.2 (inset) were printed and subsequently sintered. For this as well as all other printing trials dealt with in this chapter, a nozzle with $80 \mu \mathrm{m}$ diameter was used. These square-shaped structures comprised a single layer of printed silver. Prior to plating, they were cleaned by immersing the substrate containing these structures in a bath containing 'Neutraclean', a neutral aqueous cleaning solution, for 5 minutes at room temperature. After cleaning, the substrate was rinsed for 1 minute in de-ionised water. 
Chapter 4

The plating bath [19] comprised 78.5\% demineralised water, 12\% Envision EC $2130 \mathrm{M}$, 6\% Envision EC 2130 A, 3\% Envision EC 2130 B and $0.5 \%$ formaldehyde. It was maintained at $48{ }^{\circ} \mathrm{C}$ during plating. The outcome of the plating process that lasted 30 minutes can be understood from figure 4.2: some silver seed layers delaminated almost completely, while a few others that remained were very fragile, and they came off the substrate on slightest disturbance. Copper was deposited on these layers, but the morphology was inhomogeneous as a result of blister formation. It can be reasoned that there are 2 main causes for delamination of silver: (1) as the copper grains grew on the silver seed layers, they coalesced. This resulted in mechanical stresses on the seed layer below; and (2) the alkaline plating bath attacked the interface between the seed layer and the substrate. Since the chemical bonding between these two was not strong, and since the mechanical interlocking between them was insufficient, delamination of silver occurred due to the abovementioned causes.

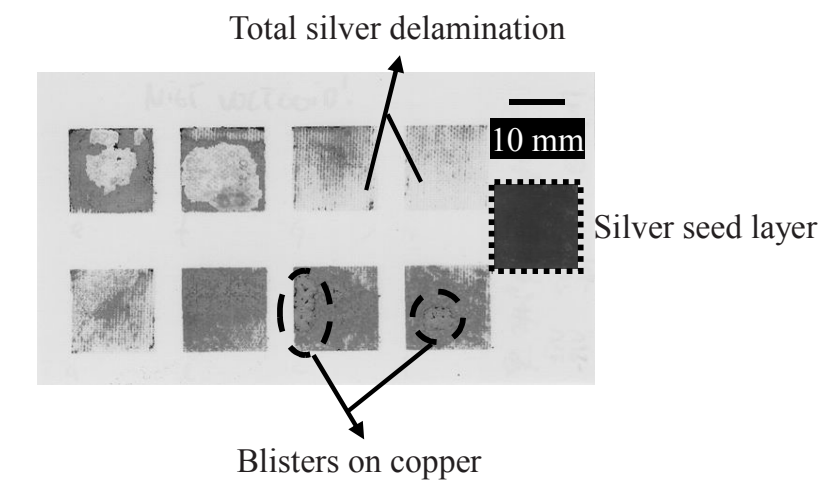

Figure 4.2: Silver seed layers on an untreated RO4003 substrate after copper plating; Inset: a silver seed layer prior to plating.

\subsubsection{Plasma treatment}

Since it was not possible to obtain robust electroless plated copper on seed layers printed on untreated RO4003, it was decided to modify the surface morphology of the substrate using plasma treatment. To find the optimal surface morphology, a DoE-based analysis was carried out. 


\subsubsection{Experimental design}

The experimental design techniques commonly used for process analysis are full factorial, fractional factorial and central composite rotatable design. The CCRD gives sufficient information to describe majority of steady-state process responses [20]. It requires much fewer runs when compared to the full factorial design and gives a clearer picture about interactions between the process variables than a fractional factorial method.

The CCRD was chosen in such a way that it contains ' $2^{\mathrm{n}}$ ' factorial treatment designs, ' $2 n$ ' axial or star points and sufficient replications at the centre of the design. Here, ' $n$ ' represents the number of process variables under study. Initial plasma etching trials showed that four factors, namely power $(\mathrm{P})$, time of exposure of the substrate to plasma $(\mathrm{t})$, flow rate of $\mathrm{O}_{2}\left(\mathrm{f}_{-} \mathrm{O}_{2}\right)$ and flow rate of $\mathrm{CF}_{4}\left(\mathrm{f}_{-} \mathrm{CF}_{4}\right)$, are the most relevant parameters that need to be studied. The operating pressure, which is generally considered important in plasma etching, could not be pre-set in the available equipment. As a result, the CCRD consisted of 16 factorial treatment designs, with 8 star points and 7 replicates of the centre point, thus 31 experiments in total. In comparison, a full factorial design for the same process would have resulted in $3^{4}=81$ experiments. Star points and replicates were added to the design to estimate the curvature and error of the model [21]. The response model for 4 variables can be expressed as [22]:

$Y=\beta_{0}+\sum_{i=1}^{4} \beta_{i} x_{i}+\sum_{i=1}^{4} \beta_{i i} x_{i}^{2}+\sum_{i=1}^{3} \sum_{j=i+1}^{4} \beta_{i j} x_{i} x_{j}$

Eqn. (4.1)

Here, $Y$ represents the yield; $\beta_{0}$ is a constant intercept; $\beta_{i}, \beta_{i i}$ and $\beta_{i j}$ represent linear, quadratic and interaction coefficients, respectively; $x_{i}$ represents the coded independent variables. The DoE model was used to understand the influence of the process parameters on the outcome of the plasma etching process. The experimental design and the coded parameter levels are listed in table 4.2; the corresponding parameter names and their actual values are listed in table 4.3. 
Chapter 4

The magnitude of the process parameters listed in table 4.3 are much higher than those found in the literature. The reason for this is that the plasma etching equipment used for this research is an industrial-scale one with a large chamber $\left(\approx 0.7 \mathrm{~m}^{3}\right)$ that necessitated these parameter values for effective etching. The range of values of the process parameters dealt with in this chapter were selected based on prior experience of the technicians at Thales Nederland B.V., a partner in this research and the owner of the said plasma equipment. In table 4.2, runs 1-16 represent the 2-level full factorial model, runs 17-24 represent the star points, and runs 25-31 represent the centre point of the model.

Table 4.2: CCRD for four coded process variables.

\begin{tabular}{|c|c|c|c|c|}
\hline Runs & $\boldsymbol{x}_{\boldsymbol{I}}$ & $\boldsymbol{x}_{2}$ & $\boldsymbol{x}_{\boldsymbol{3}}$ & $\boldsymbol{x}_{\boldsymbol{4}}$ \\
\hline 1 & -1 & -1 & -1 & -1 \\
2 & 1 & -1 & -1 & -1 \\
3 & -1 & 1 & -1 & -1 \\
4 & 1 & 1 & -1 & -1 \\
5 & -1 & -1 & 1 & -1 \\
6 & 1 & -1 & 1 & -1 \\
7 & -1 & 1 & 1 & -1 \\
8 & 1 & 1 & 1 & -1 \\
9 & -1 & -1 & -1 & 1 \\
10 & 1 & -1 & -1 & 1 \\
11 & -1 & 1 & -1 & 1 \\
12 & 1 & 1 & -1 & 1 \\
13 & -1 & -1 & 1 & 1 \\
14 & 1 & -1 & 1 & 1 \\
15 & -1 & 1 & 1 & 1 \\
16 & 1 & 1 & 1 & 1 \\
17 & -2 & 0 & 0 & 0 \\
18 & 2 & 0 & 0 & 0 \\
19 & 0 & -2 & 0 & 0 \\
20 & 0 & 2 & 0 & 0 \\
21 & 0 & 0 & -2 & 0 \\
22 & 0 & 0 & 2 & 0 \\
23 & 0 & 0 & 0 & -2 \\
24 & 0 & 0 & 0 & 2 \\
$25-31$ & 0 & 0 & 0 & 0 \\
\hline
\end{tabular}


Table 4.3: Actual values of the process variables.

\begin{tabular}{|c|c|c|c|c|}
\hline Code & $\begin{array}{c}\mathbf{P}\left(\boldsymbol{x}_{1}\right) \\
\text { watt }\end{array}$ & $\begin{array}{c}\mathbf{t}\left(\boldsymbol{x}_{2}\right) \\
\boldsymbol{m i n}\end{array}$ & $\begin{array}{c}\mathbf{f}_{-} \mathbf{O}_{2} \\
\left(\boldsymbol{x}_{3}\right) \\
\boldsymbol{m l} / \boldsymbol{m i n}\end{array}$ & $\begin{array}{c}\mathbf{f}_{-} \mathbf{C F}_{4} \\
\left(\boldsymbol{x}_{4}\right) \\
\boldsymbol{m l} / \mathbf{m i n}\end{array}$ \\
\hline-2 & 2500 & 10 & 0 & 0 \\
-1 & 2900 & 20 & 500 & 50 \\
0 & 3300 & 30 & 1000 & 100 \\
1 & 3700 & 40 & 1500 & 150 \\
2 & 4100 & 50 & 2000 & 200 \\
\hline
\end{tabular}

As per the experimental design, 31 substrates, each measuring $100 \mathrm{~mm} \times 100 \mathrm{~mm}$, were cut and subsequently plasma etched. During this etching process, the specimen i.e. substrate is immersed in plasma-containing gases that react with it. At relatively high process pressures of more than 0.2 mbar, the mechanism for etching is predominantly chemical, and the physical bombardment is minimal; the chemical reactions are promoted by radicals in $\mathrm{O}_{2}$ and $\mathrm{CF}_{4}$ [23]. For the chosen flow rates, the operating pressure was above 0.2 mbar for all the experimental runs. The addition of $\mathrm{CF}_{4}$ to an oxygen plasma greatly increases the etch rate. Even though oxygen is the etchant for polymers, atomic fluorine creates radicals at the surface of the polymer for further attack by oxygen [24]. The process temperature is a complex function of power input and heat transfer phenomena, and was not controlled during the experimental runs. The substrate temperature (maximum $88^{\circ} \mathrm{C}$ ), indicated by a digital readout on the plasma equipment during all the runs was well below the glass transition temperature $\left(\mathrm{T}_{\mathrm{g}}\right)$ of the substrate material, which, according to the supplier, is greater than $280^{\circ} \mathrm{C}$; hence, it was not expected to play a major role in the outcome of the etching process.

\subsubsection{Post-processing of plasma treated substrates}

After etching, the contact angle of water on these substrates was measured with the purpose of calculating the surface energy of the latter, using the Neumann's equation of state, given below [25]. This approach is one of many that are available.

$\cos \theta=2 \sqrt{\frac{\gamma_{S}}{\gamma_{L}}} e^{-\beta\left(\gamma_{L}-\gamma_{S}\right)^{2}}-1$

Eqn. (4.2) 
where $\theta$ is the contact angle (degree), $\gamma_{S}$ is the surface free energy of substrate $\left(\mathrm{J}^{-\mathrm{m}^{-2}}\right), \gamma_{L}$, the surface tension of the liquid i.e. water $\left(\mathrm{N} \cdot \mathrm{m}^{-1}\right)$, and $\beta$, a constant with a value of 1.247E-04. The surface tension of water used for the calculations was $72.75 \mathrm{mN} \cdot \mathrm{m}^{-1}$.

The next step was the surface roughness measurement, using a DEKTAK surface profiler. The radius of the stylus fitted to this surface profiler was $5 \mu \mathrm{m}$. Subsequent to surface characterisation, rectangular test patterns with arbitrarily determined dimensions $(30 \mathrm{~mm} \times 10 \mathrm{~mm})$ were inkjet printed on these substrates. The thickness of the patterns was highly dependant on surface roughness and surface energy of the individual substrates, and was difficult to characterise due to the pronounced roughness of certain substrates. Measurements on selected substrates after sintering of the test patterns indicated that the thicknesses were in the order of $1 \mu \mathrm{m}$. Scotch tape tests were done on these patterns to qualitatively rank the adhesive strength of the substrates under study. The spreading of the ink on the substrates was also studied for consistency of the droplet edges and the extent of spreading. For this purpose, a micropipette was used to deposit ink droplets with a constant volume of $20 \mu$. The reason behind deposition of such a large droplet, the diameter of which was nearly 20 times that of a droplet from the inkjet printer, was to make the ink cover a larger area on the substrate, to keep the influence of local roughness peaks and valleys minimal. Prior experience showed that the roughness peaks and valleys on a roughened substrate's surface can be several times the thickness of an inkjet printed droplet upon spreading on the substrate. A local cluster of roughness peaks or valleys will radically modify the spreading behaviour of such a droplet. However, scaling effects due to the increase in droplet size were not investigated.

From the results of the Scotch tape tests, surface roughness as well as surface energy measurements and droplet spreading studies, substrates with optimal surface morphology were selected. For a given material, a substrate with high surface energy provides better adhesive strength than one with lower surface energy, by decreasing the contact angle of the deposited liquid, thereby increasing the interfacial area. An increase in the interfacial area results in more opportunities for chemical bond formation. Mechanical interlocking resulting from surface roughness is also enhanced by larger interfacial area. On the other hand, if the surface is too rough, it is not possible to inkjet 
print with accuracy. The edge as well as the cross-sectional accuracy of printed structures is very important for (RF-) electronic applications, especially in the gigahertz frequency range dealt with in this thesis. For this reason, it was attempted to select substrates that are sufficiently rough, but not too rough, and have high enough surface energy, so that the printed droplet does not bead up with a high contact angle.

\subsubsection{Inkjet printing-electroless plating trials after plasma treatment}

Silver test structures were inkjet printed on selected RO4003 substrates, and were subsequently electroless copper plated. This was done to ascertain whether or not the surface modification by plasma treatment enables delamination-free electroless copper plating on inkjet printed seed layers. Finally, Scotch tape tests were done on these structures to qualify their adhesion.

\subsection{Results and discussion}

\subsubsection{Plasma etching}

The measured contact angles and the corresponding surface energy values on plasma treated substrates are depicted in figure 4.3. In figure 4.4, the measured surface roughness values in terms of $R_{a}$ are plotted. Specimen number 0 indicates the untreated RO4003 series substrate. The numbers of the substrates correspond to the runs as listed in table 4.2. It is interesting to note that three substrates with the highest surface roughness (numbers 7,8 and 22) also have surface energy values that are among the highest measured. This is perhaps logical, as a surface with pronounced surface roughness has a larger exposed area; to create such an area, more bonds between the molecules of the solid would have been broken than for a smoother surface [26] and hence the higher surface energy. That said, this observation can also be attributed to the complete exposure of silica fillers present in the substrate material, due to the complete etching off of the polymer top layer of these substrates. The surface energy of silica is greater than that of the polymeric matrix material. 


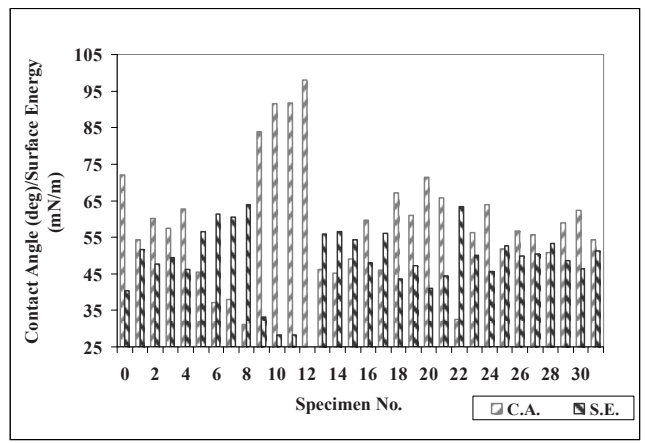

Figure 4.3: Contact angle (C.A.) and surface energy (S.E.) values of the plasma treated substrates.

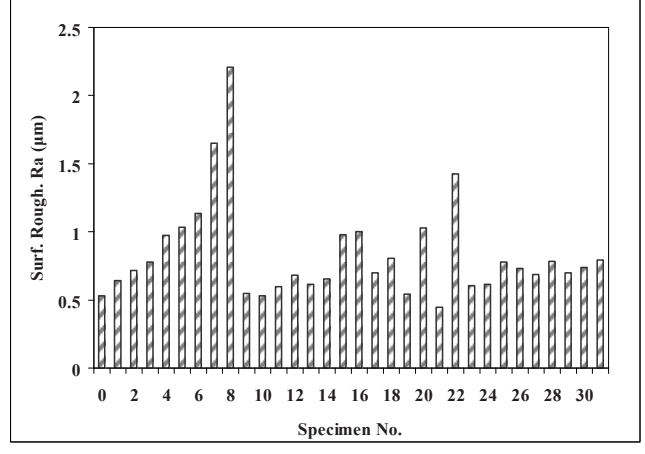

Figure 4.4: Surface roughness $\left(\mathrm{R}_{\mathrm{a}}\right)$ values of the plasma treated substrates.

Figure 4.5 shows a scatter plot of surface roughness $\left(\mathrm{R}_{\mathrm{a}}\right)$ versus contact angle and surface energy. As seen clearly in this graph, low surface roughness values yield high contact angles, and high surface roughness values result in low contact angles. Similarly, the surface energy, by and large, increases with surface roughness. However, the relationship between surface roughness and these 2 physical quantities is too complex to be derived based on these observations alone. Even though an increase in surface roughness of the substrate results in a higher contact area between the substrate and the ink, resulting in lower contact angles, there are deviations as seen in figure 4.5 , since the substrate material contains more than one phase. This complexity due to the composition of the substrate material is discussed in detail later in this section. 


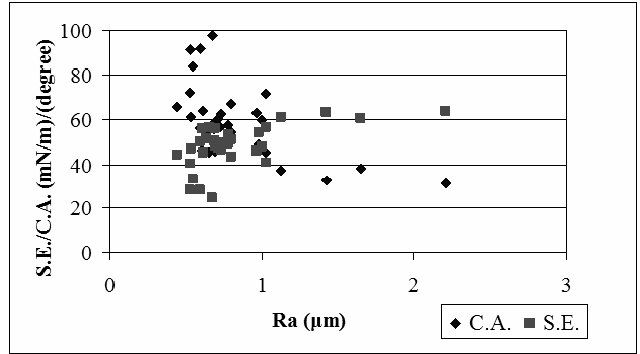

Figure 4.5: Surface roughness $\left(\mathrm{R}_{\mathrm{a}}\right)$ versus surface energy (S.E.) and contact angle (C.A.).

Table 4.4: Ranking-based system for assessing the substrate characteristics with respect to adhesion and droplet spreading.

\begin{tabular}{|c|c|c|}
\hline Substrate & Scotch tape & Droplet \\
\hline 0 & - & 0 \\
1 & - & + \\
2 & - & + \\
3 & - & + \\
4 & + & + \\
5 & + & + \\
6 & 0 & 0 \\
7 & + & - \\
8 & + & - \\
9 & - & - \\
10 & - & - \\
11 & - & - \\
12 & + & 0 \\
13 & + & + \\
14 & + & + \\
15 & + & + \\
16 & + & + \\
17 & 0 & + \\
18 & 0 & + \\
19 & - & + \\
20 & + & - \\
21 & - & - \\
22 & + & - \\
23 & - & + \\
24 & - & \\
$25-31$ & + & - \\
& & \\
\hline
\end{tabular}

In order to analyse and classify the results of the adhesion test and droplet spreading studies, a ranking-based system was formulated. The reason for developing such a system is that both these studies were qualitative - adhesion using the scotch tape test 
Chapter 4

and subsequent visual assessment, and droplet spreading by visual assessment. The criterion for droplet spreading was not just the contact angle, but also the smoothness of the edge of the droplet after spreading. Table 4.4 shows the results of these studies.

In table 4.4, '+' represents a favourable response, ' 0 ' represents a moderately favourable response and '-' represents an unfavourable response. It is important to note that for all the scotch tape tests, some material was peeled off the printed silver layer. The type of failure was cohesive and not adhesive. The reason for this is that since all the substrates were roughened albeit with varying magnitudes by the plasma treatment, the resulting mechanical interlocking averts an adhesive failure, thereby giving rise to a cohesive failure. A detailed description of adhesion mechanisms is given in chapter 5 .

For the adhesion test, a ' + ' represents minimal peel off of the printed silver structure; a ' 0 ' represents a greater amount of material removed when compared to '+', and a '-' represents almost complete peel off. Figure 4.6 shows the processed images of three peeled off tapes, representing the three classifications. They were processed using the image processing software program ImageJ. Similarly, for the droplet spreading, a ' + ' represents controlled spreading of the droplet with no beading-up and regular (smooth) edge; a ' 0 ' represents controlled spreading of the droplet with either partial beading-up or unsmooth edges, and a '-' indicates either pronounced beading-up or uncontrolled spreading of the droplet. Figure 4.7 shows three substrates on which the ink droplets deposited using the micropipette demonstrate the abovementioned spreading behaviours. 

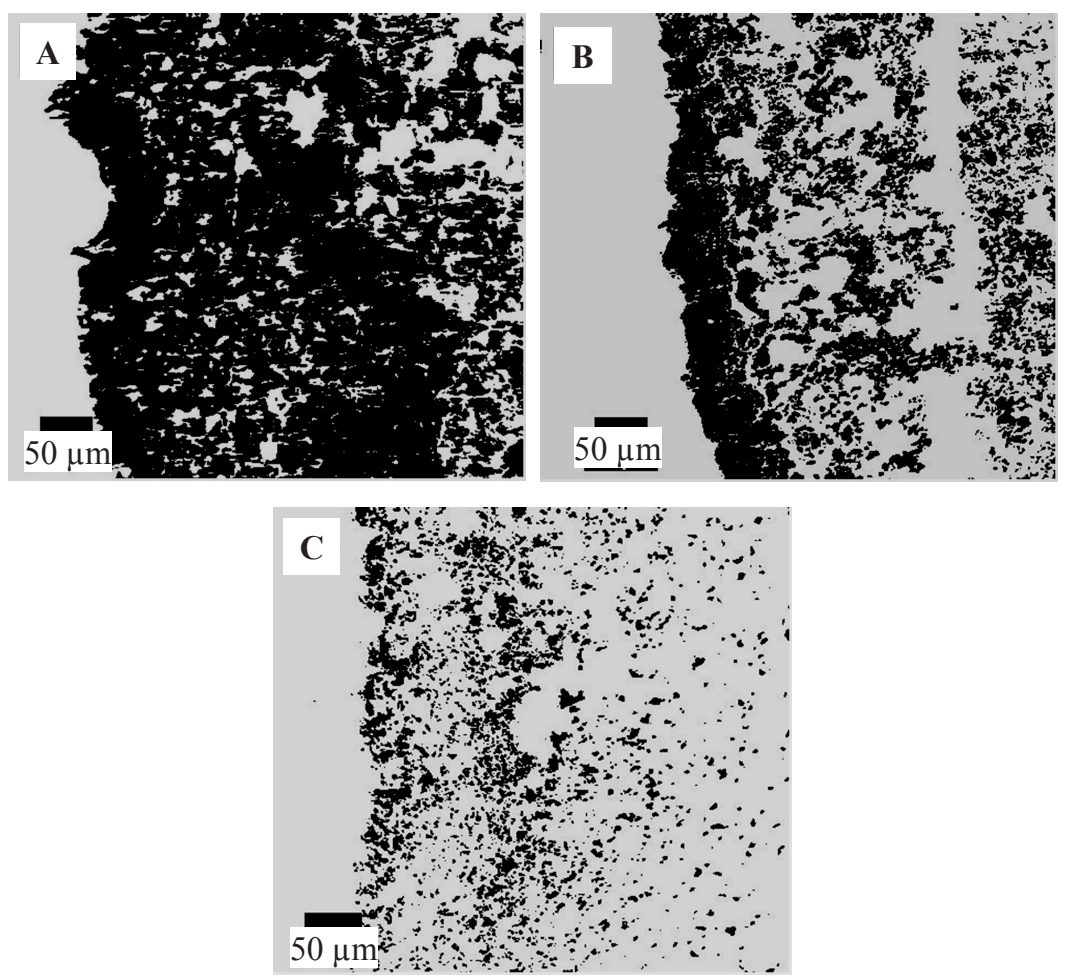

Figure 4.6: Processed images of peeled off scotch tapes representing (A) almost complete peel off $(-),(B)$ average peel off $(0)$ and $(C)$ minimal peel off $(+)$.

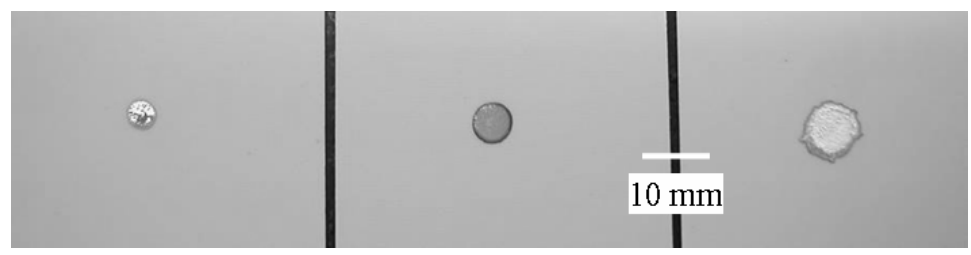

Figure 4.7: Substrates with different droplet spreading characteristics viz. (from left to right): beading-up (-), controlled spreading $(+)$ and controlled spreading with unsmooth edges $(0)$.

It can be seen from table 4.4 that substrates 4, 5, 14, 15, 16 and 20, along with substrates 25 to 31 , exhibit a favourable behaviour and meet the requirements mentioned before. Since substrates 25 to 31 represent the centre point of the DoE model, they were all subjected to the same plasma treatment parameter set. So, it is not surprising that all of them exhibit same or similar properties. The surface energy values of these substrates are on the higher side $\left(>45 \mathrm{mNm}^{-1}\right)$ indicating favourable droplet spreading, and their $\mathrm{R}_{\mathrm{a}}$ 
Chapter 4

values are in the order of $1 \mu \mathrm{m}$, which, incidentally, corresponds to the layer thickness of the inkjet printed test structures.

\subsubsection{Substrate selection}

From the results of adhesion tests, roughness and surface energy measurements, as well as droplet spreading studies, a logical conclusion will be to select one or more of the substrates highlighted in table 4.4 as optimal one(s). However, SEM observations of these surfaces revealed a problem: it was mentioned before that the substrate material is composite, with a thermoset matrix, glass fibres and silica fillers. The plasma etching process etched away either partially or completely the top polymer layer of the substrates highlighted in table 4.4, exposing the silica fillers. Especially affected were substrates 7, 8 and 22, which had the highest surface roughness and surface energy values - there were no visible remnants of the polymer top layer on their surface after etching. The removal of the polymer top layer would have some influence on the dielectric behaviour of the substrates, which would in turn affect their RF performance especially at high frequencies.

SEM images of an unetched RO4003 substrate and a plasma etched RO4003 substrate (number 18), shown in figure 4.8, reveal the etching away of polymer top layer of the latter.
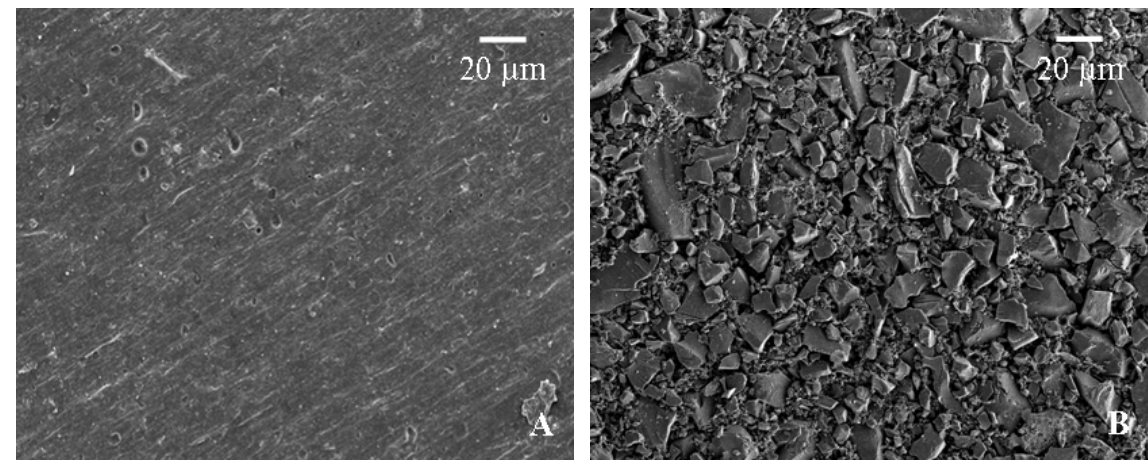

Figure 4.8: (A) An unetched RO4003 substrate and (B) a plasma etched RO4003 substrate based on DoE parameter set number 18 . 
It became clear from the SEM observations that one or more of the plasma treatment parameters are too high in magnitude and that a compromise has to be found concerning the substrate characteristics (namely surface roughness and surface energy), test results (namely adhesion testing and droplet spreading), and the extent of etching. SEM observations indicated that only the substrates numbered 1, 10 and 19 had their polymer top layer intact, with only the roughening of the polymer resulting from the plasma treatment. While the surface roughnesses of all these substrates were similar, only substrates 1 and 19 had high surface energy $\left(>45 \mathrm{mN} \cdot \mathrm{m}^{-1}\right)$. Substrate 10 had a very low surface energy due to the higher proportion of $\mathrm{CF}_{4}$ in the gas mixture. Detailed explanation concerning the influence of $\mathrm{CF}_{4}$ as well as the other process parameters on etching is provided in the next section.

Owing to their lower surface roughnesses when compared with those of the substrates that are highlighted in table 4.4, substrates 1, 10 and 19 all demonstrated poor adhesion with the printed silver layer, confirmed by the '-' ratings in the ranking system. This lead to a conundrum: on the one hand are the substrates highlighted in table 4.4, with good adhesion performance and good droplet spreading behaviour, but with surface morphology that would affect RF performance; on the other hand are substrates 1,10 and 19 with good surface morphology that will have minimal effect on RF performance, but with poor adhesion behaviour. After deliberation about the problems and the prospects of all these substrates, it was decided that the latter substrates i.e. those with good surface morphology but relatively poor adhesion are better for high frequency RF applications. Even more specifically, it was decided to select substrates 1 and 19, as substrate 10 had a very low surface energy leading to a high contact angle of the deposited droplet. The SEM images of substrates 1 and 19 are shown in figure 4.9. 
Chapter 4
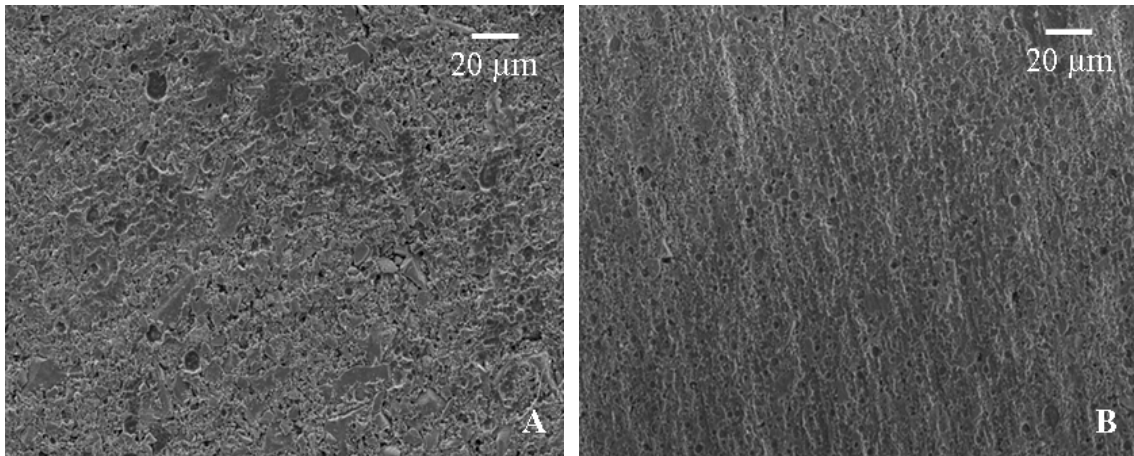

Figure 4.9: SEM images of $(A)$ substrate 1 and $(B)$ substrate 19.

The increase of surface roughness from an unetched substrate $(0.5 \mu \mathrm{m})$ to the selected etched substrates $(0.64 \mu \mathrm{m}$ for substrate 1 and $0.56 \mu \mathrm{m}$ for substrate 19$)$ is only marginal. However, even this marginal increase proved to be sufficient for the electroless plating process. Silver seed layers on RO4003 substrates that were plasma treated with the same parameter sets as substrates 1 and 19 were able to withstand the process-generated stresses resulting from the growth of copper, and were hence plated without problems. The increased anchoring sites i.e. roughness profiles per unit area of plasma treated substrates is an important contributor to the success of the plating process. Figure 4.10 depicts the surface topography of untreated and treated substrates at a high magnification.

The contribution of enhanced chemical bonding due to the functional groups created on the surface of the polymer due to plasma etching also played an important role in improving adhesion. The surface energy of the etched substrates $\left(51.5 \mathrm{mN} \cdot \mathrm{m}^{-1}\right.$ for substrate 1 and $47.2 \mathrm{mN} \cdot \mathrm{m}^{-1}$ for substrate 19) showed a noticeable increase when compared to the untreated substrate $\left(40.4 \mathrm{mN} \cdot \mathrm{m}^{-1}\right)$. Scotch tape adhesion tests done on printed-plated structures on these substrates showed that there was neither cohesive nor adhesive failure. 

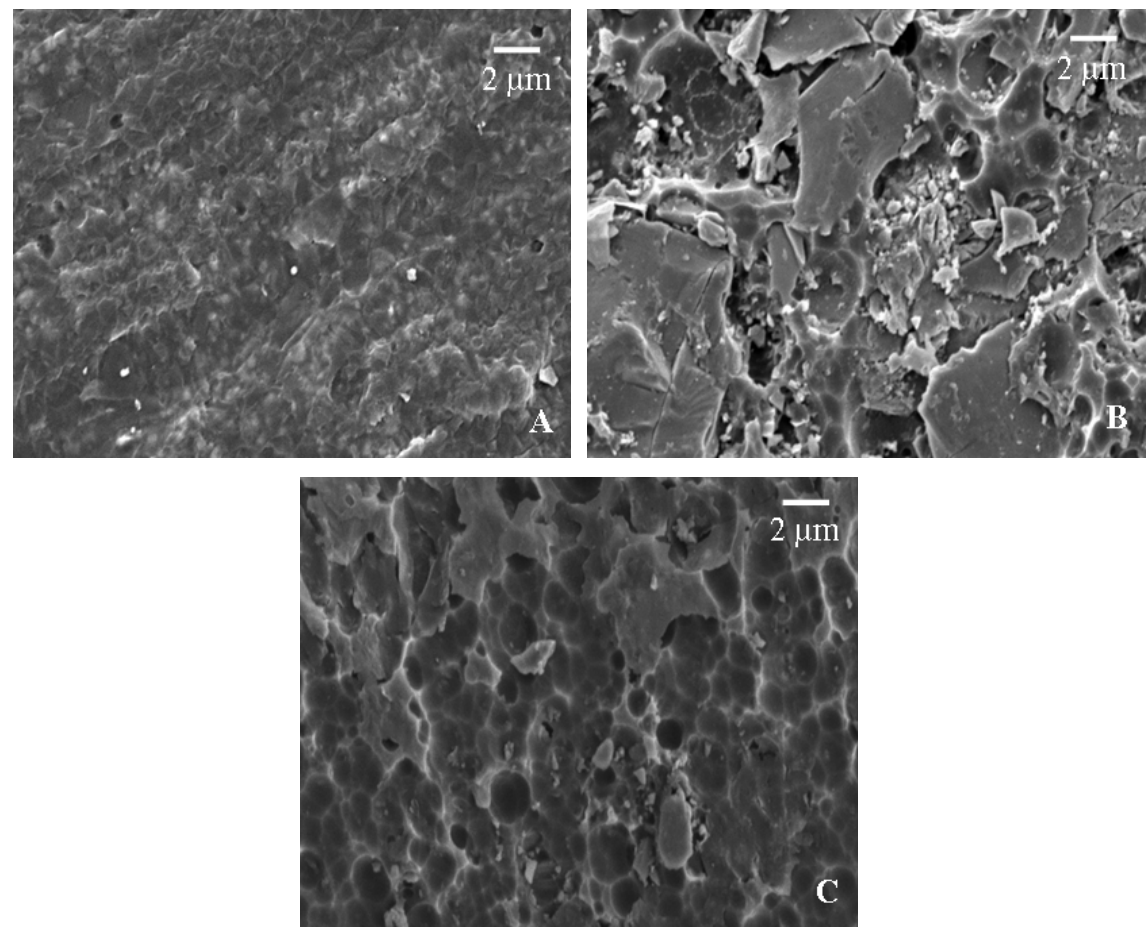

Figure 4.10: High magnification micrographs of the surface topography of (A) untreated substrate, $(B)$ substrate 1 and $(C)$ substrate 19.

\subsubsection{Analysis of the DoE model}

The experimental responses of the DoE were analysed using MINITAB15 statistical software. The key formulae used by the software in analysing the model are listed in Appendix- $A$. The model equations for the two variables under study, namely surface roughness and surface energy, were derived based on eqn. 4.1 .

\section{Surface roughness model:}

$Y=0.643+0.099 x_{1}+0.168 x_{2}+0.32 x_{3}-0.19 x_{4}+0.034 x_{1}{ }^{2}+0.044 x_{2}{ }^{2}+0.131 x_{3}{ }^{2}+0.011 x_{4}{ }^{2}$ $+0.042 x_{1} x_{2}+0.075 x_{1} x_{3}-0.101 x_{1} x_{4}+0.138 x_{2} x_{3}-0.16 x_{2} x_{4}-0.235 x_{3} x_{4}$

Eqn. (4.3) 
Chapter 4

Surface energy model:

$$
\begin{aligned}
& Y=50.32-1.43 x_{1}-1.35 x_{2}+7.6 x_{3}-5.05 x_{4}-0.27 x_{1}{ }^{2}-1.7 x_{2}{ }^{2}+0.73 x_{3}{ }^{2}-0.77 x_{4}{ }^{2} \\
& -0.65 x_{1} x_{2}+0.91 x_{1} x_{3}-1.21 x_{1} x_{4}+0.81 x_{2} x_{3}-1.13 x_{2} x_{4}+3.59 x_{3} x_{4}
\end{aligned}
$$

Eqn. (4.4)

In these models, $x_{1}, x_{2}, x_{3}$ and $x_{4}$ represent the process variables listed in table 4.3. Substituting the coded values of the process variables gives the yield in terms of $\mu \mathrm{m}$ for eqn. 4.3 and $\mathrm{mN} \cdot \mathrm{m}^{-1}$ for eqn. 4.4 . It is clear from eqn. 4.3 that the $\mathrm{O}_{2}$ flow rate has the strongest effect on surface roughness, as it has the highest $\beta$ coefficient. Positive sign of this coefficient indicates that the surface roughness will increase with increasing $\mathrm{O}_{2}$ flow rate. The $\mathrm{CF}_{4}$ flow rate also has strong effect, albeit a negative one. As the $\mathrm{CF}_{4}$ flow rate increases, the surface roughness values tend to be lower, even at high $\mathrm{O}_{2}$ flow rates. This phenomenon can be explained by fact that high concentration of fluorine radicals results in passivation via the formation of a fluorinated surface layer, which inhibits attack i.e. etching by oxygen [27]. The interaction of the two flowrates is significant, as the percentage of $\mathrm{CF}_{4}$ in $\mathrm{O}_{2}$ is a key factor in determining the extent of etching. The analysis also revealed that the effect of power and time on surface roughness is not significant for the practical range of parameter values studied. The other terms in the model are also insignificant. The significance and the insignificance of the process parameters were ascertained based on a null hypothesis ${ }^{1}$ with an $\alpha$-level ${ }^{2}$ of 0.05 . However, for a slightly increased $\alpha$-level of 0.07 , time becomes significant. So, insignificance does not mean an absence of influence - it is just that the influence of an insignificant parameter on the process outcomes is not as prominent as that of a significant parameter.

As far as the surface energy is concerned, the $\mathrm{O}_{2}$ flow rate again has the strongest effect, as seen from eqn. 4.4. In this case also, $\mathrm{CF}_{4}$ flow rate has a strong negative effect due to its contribution towards the formation of the surface passivation layer. The interaction

\footnotetext{
${ }^{1}$ Null hypothesis is one in which the initial claim is assumed to be true. Here, the initial claim is that the process parameters do not influence the outcomes.

${ }^{2}$ Level of significance; it was chosen based on the most commonly used level. With an $\alpha$-level of 0.05 , the probability of finding an effect that does not exist is only $5 \%$, which is acceptable under most situations. If the 'p' value of a process parameter is greater than this level, the null hypothesis cannot be rejected. On the other hand, if the ' $p$ ' value is less than or equal to the chosen $\alpha$-level, the null hypothesis can be rejected [28].
} 
effect of the flow rates of these gases are, understandably, very important as well. The influence of power and time, as well as other terms in the model are insignificant.

The ANOVA (analysis of variance) showed that there is a significant lack-of-fit in the models and that they contain a few observations with large residuals. The $\mathrm{R}^{2}$ values (calculated using the equation listed in Appendix- $A$ ) of both the models represented in equations (3) and (4), are about $85 \%$. Even though these $\mathrm{R}^{2}$ values are acceptable, they do indicate that the error component i.e. the amount of variation in the response data that is not explained by the predictors (process parameters) in the regression model is not negligible. Predicted $\mathrm{R}^{2}$ values, which indicate how well the model can predict future outcomes (responses), are very low: 12\% for surface roughness and $31 \%$ for contact angle. The inability of the predictors to explain the amount of variation in the response data and the very low predicted $\mathrm{R}^{2}$ values can be due to the following influences:

(1) The presence of different phases in the substrate (thermoset polymer, silica and glass fibre) has a major effect on $\mathrm{R}^{2}$ values: SEM images revealed that after plasma etching, a number of substrates had their polymer top layer completely etched away, revealing silica and, in some cases, glass fibres present underneath. Consider the surface roughness model represented by equation (3): the measured surface roughness i.e. the observed responses might represent the roughness of the polymer top layer for a particular measurement, whereas for another measurement, it might represent the roughness of the exposed silica or glass fibre, or a combination of these materials. In this case, the effect of the etching parameters is difficult to quantify. The presence of probable outliers in both the models, as shown in figure 4.11 depicting standardized residuals ${ }^{3}$ supports this explanation. An outlier is an observation with a large residual value. In layman terms, an outlier is a point in the residual plot that is far away from zero. The probable outliers are encircled in figure 4.11 .

(2) The influence of the operating pressure inside the plasma equipment could be pronounced. Since it could not be pre-set and hence left out of the DoE model, its influence was not quantified. Even though the operating pressures were recorded

\footnotetext{
${ }^{3}$ Standardized residuals pave way for the comparison of all the residuals on a standard scale. Values between -1 and +1 are usual, between -2 and +2 indicate something unusual (with respect to the process response), and between -3 and +3 indicate something out-of-the-ordinary [29].
} 
Chapter 4

during each plasma treatment cycle, including these values in the DoE model will not reveal the influence of this parameter on the process outcomes, as operating pressure is a dependant parameter in this case i.e. the magnitude of operating pressure was governed by the flowrates of the gases. In order to ascertain the influence of the operating pressure, a plasma etching setup with independent pressure regulation is needed.

(3) Although all the substrates subjected to plasma treatment were machined from a single large substrate, there were local variations in the initial roughness values and profiles. The variations resulted in dissimilar initial surface conditions among the substrates. Since the final roughness profiles produced by the plasma treatment also depend on the initial substrate surface condition, the local variations may have also contributed to the low predicted $\mathrm{R}^{2}$ values and not-so-high $\mathrm{R}^{2}$ values.
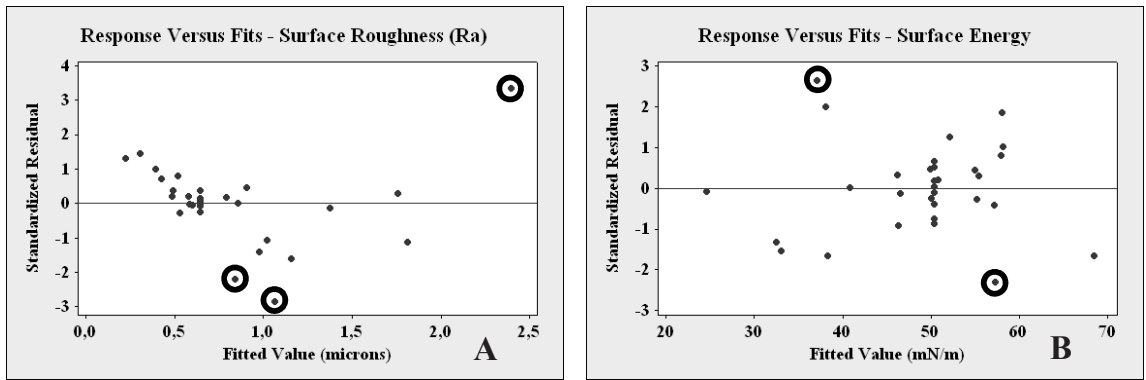

Figure 4.11: Response versus fits plotted against (A) surface roughness and (B) surface energy.

Surface plots and contour plots are generally used to explain the effects of process parameters on process outcomes. In both plot types, two process parameters are varied, the others fixed at a specific level (hold value) and the process outcomes are analysed. In this chapter, contour plots have been preferred due to their straightforward representation and the absence of perspective (unlike the surface plots), which might have to be varied based on the shape of the profile of the plot. Since the DoE in discussion has 4 process parameters, each contour plot was plotted by varying two of them and keeping the other two at mid-level i.e. at ' 0 ' level. The actual values of the levels specified in the plots are found in table 4.3. The important contour plots from the DoE analyses are depicted in figures 4.12 and 4.13 , with the former representing the dependence of surface roughness $\left(\mathrm{R}_{\mathrm{a}}\right)$ and the latter, that of surface energy. 

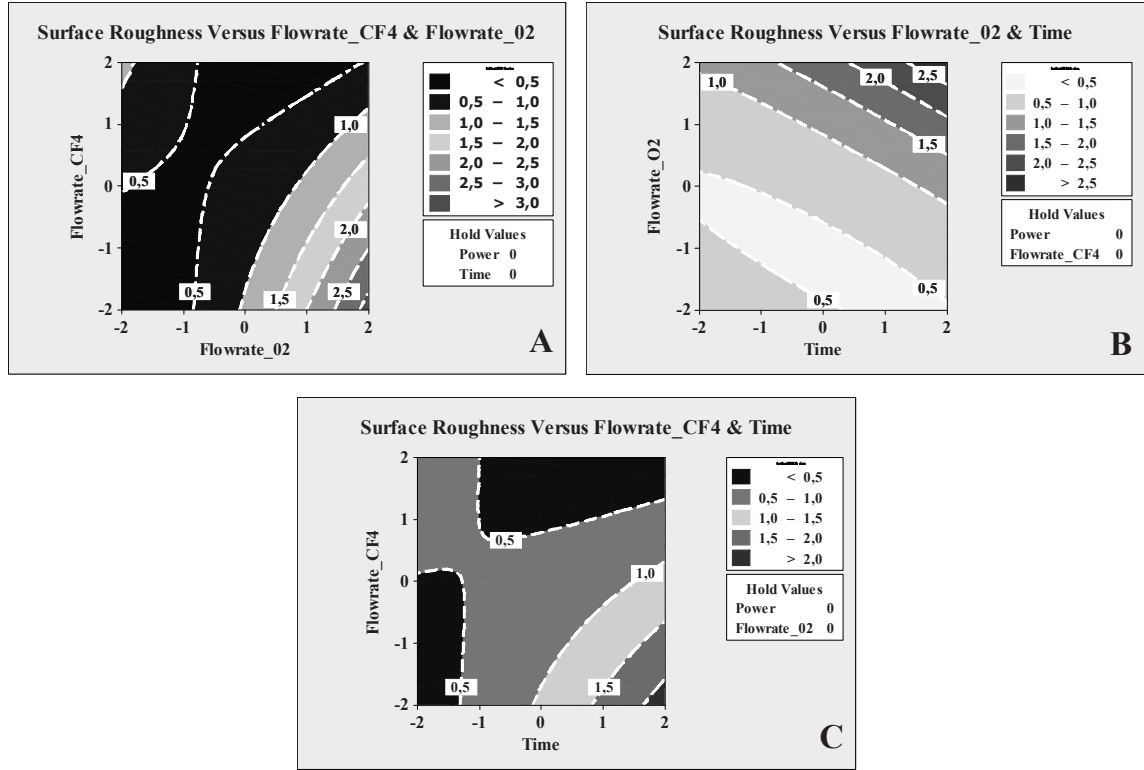

Figure 4.12: Contour plots depicting the relationship between the significant process parameters and the surface roughness of the plasma etched substrates.
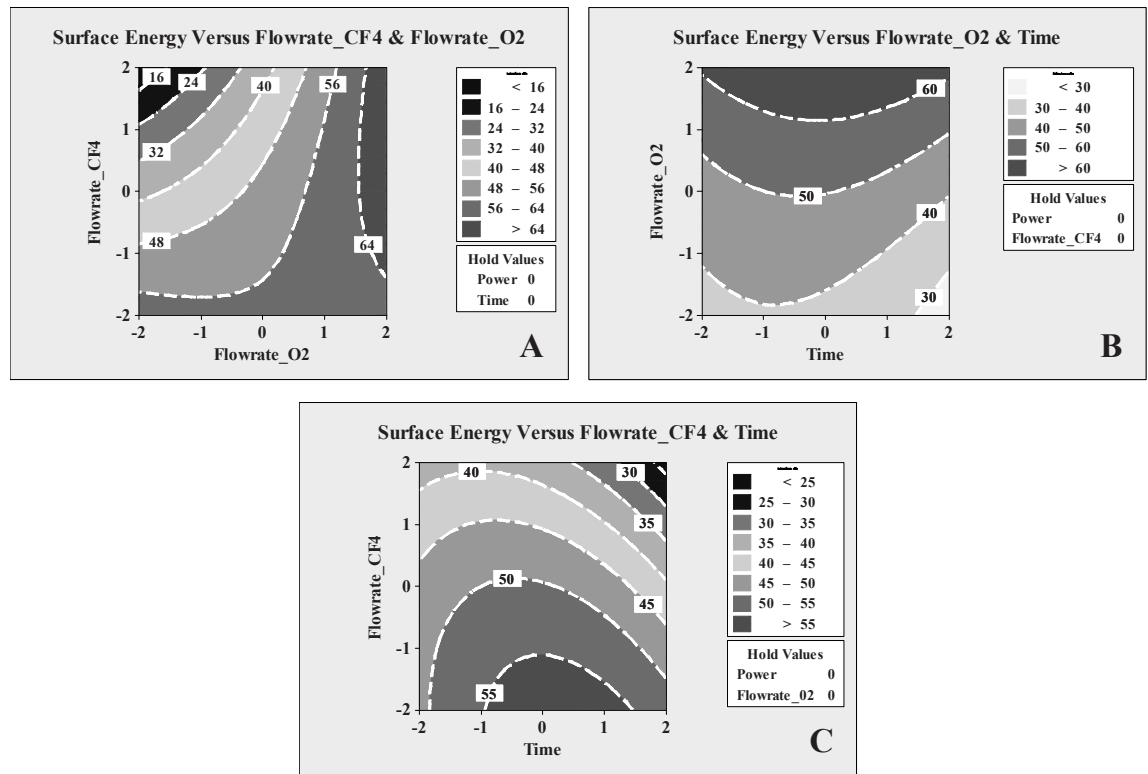

Figure 4.13: Contour plots depicting the relationship between the significant process parameters and the surface energy of the plasma etched substrates 
Chapter 4

From the contour plots of surface roughness shown in figure 4.12 and surface energy shown in figure 4.13 , the conclusions listed below can be arrived at. It should be noted that conclusions drawn here are relevant to the corresponding hold values of the parameters that are not plotted; in all the individual plots of figures 4.12 and 4.13 , the hold values are at level 0 :

(1) Figure 4.12(A): The surface roughness increases with the increase of $\mathrm{O}_{2}$ flowrate, except when the flowrate of $\mathrm{CF}_{4}$ also increases. As discussed already, the increase of the latter leads to the formation of a passivation layer on the substrate. Even though $\mathrm{CF}_{4}$ is important for high etch rates (as it promotes oxygen attack on the substrate), very low or the total absence of $\mathrm{CF}_{4}$ does not seem to influence the roughening of the substrate $-\mathrm{O}_{2}$ flowrate seems to have the decisive influence. Etch rates were not measured during this study.

(2) Figure 4.12(B): The surface roughness increases with a simultaneous increase in $\mathrm{O}_{2}$ flowrate and process time. However, at low $\mathrm{O}_{2}$ flowrates i.e. level -1 or -2 , the surface roughness values remain low irrespective of longer process times, due to a relatively higher proportion of $\mathrm{CF}_{4}$ as well as insufficient oxygen in the gas mixture. Even at shorter process times, the surface roughness is on the higher side for high $\mathrm{O}_{2}$ flowrates, reiterating the importance of this parameter in determining the surface roughness.

(3) Figure 4.12(C): At low $\mathrm{CF}_{4}$ flowrates, the surface roughness increases with increase in time, as a result of the $\mathrm{O}_{2}$ flowrate being held constant at 0 level, which is sufficient. At high $\mathrm{CF}_{4}$ flowrates, there is some roughening of the substrate when process times are lower. With increasing process time, the formation of passivation layer completely inhibits the attack of $\mathrm{O}_{2}$ on the substrate, and hence the low surface roughness values as depicted in this figure.

(4) Figure 4.13(A): Surface energy increases with increasing $\mathrm{O}_{2}$ flowrate. Unlike surface roughness, at high $\mathrm{O}_{2}$ flowrates, surface energy does not seem to be affected with increasing $\mathrm{CF}_{4}$ flowrate. But at lower $\mathrm{O}_{2}$ flowrates, high proportion of $\mathrm{CF}_{4}$ is detrimental to surface energy.

(5) Figures 4.13(B) \& 4.13(C): The influence of time on surface energy is minimal, as the latter is mainly determined by the flowrates of $\mathrm{O}_{2}$ and $\mathrm{CF}_{4}$. Just as in the case of surface roughness, at high $\mathrm{CF}_{4}$ flowrates, process time does have some influence on surface energy - for the given fixed $\mathrm{O}_{2}$ flowrate (level 0), 
a combination of long process times and high $\mathrm{CF}_{4}$ flowrates result in low surface energy values, albeit not the lowest. The lowest surface energy values are encountered when $\mathrm{O}_{2}$ flowrate is low or totally absent, as shown in figure 4.13(A).

\subsubsection{Electroless plating trials on plasma treated substrates}

The selected inkjet printed silver seed layers on plasma treated substrates were successfully copper plated. The plasma treatment parameters were the same as the ones chosen based on the DoE. Figure 4.14 shows the time evolution of copper growth on inkjet printed silver seed layers. It can be seen clearly from this figure that the copper grains grow in size with time. Microscopic studies revealed that individual copper grains reach out to the neighbouring grains, and with time, they form a continuous structure. This covers up the pores and the cracks on the inkjet printed silver seed layer.
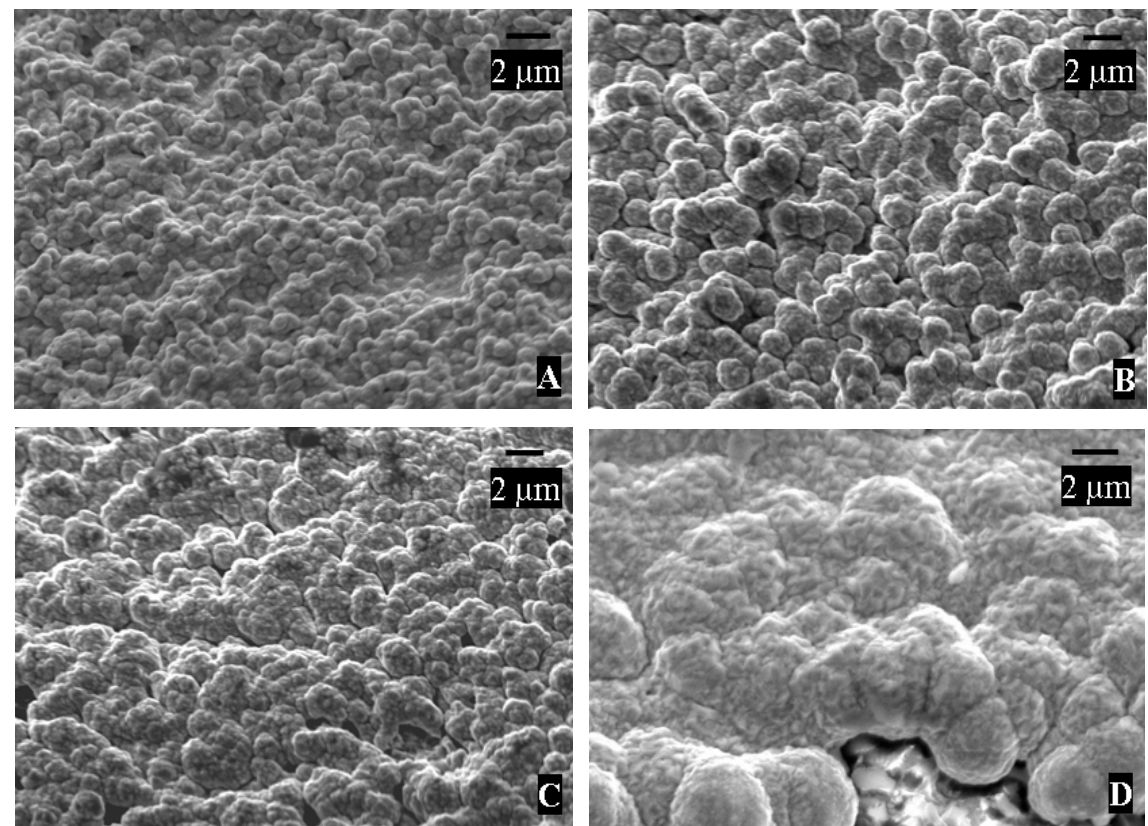

Figure 4.14: Time evolution of copper growth on silver seed layers, after (A) 2 minutes, (B) 20 minutes, (C) 30 minutes and (D) 120 minutes. 
Chapter 4

SEM studies showed that the plated copper layer was continuous, indicating that there was no local silver delamination. Figure 4.15(A) depicts an inkjet printed-electroless plated structure on a plasma treated substrate, and 4.15(B) depicts the surface morphology of the same. The final printed-plated structure on plasma treated substrates passed the Scotch tape test, as neither cohesive nor adhesive failure was observed.
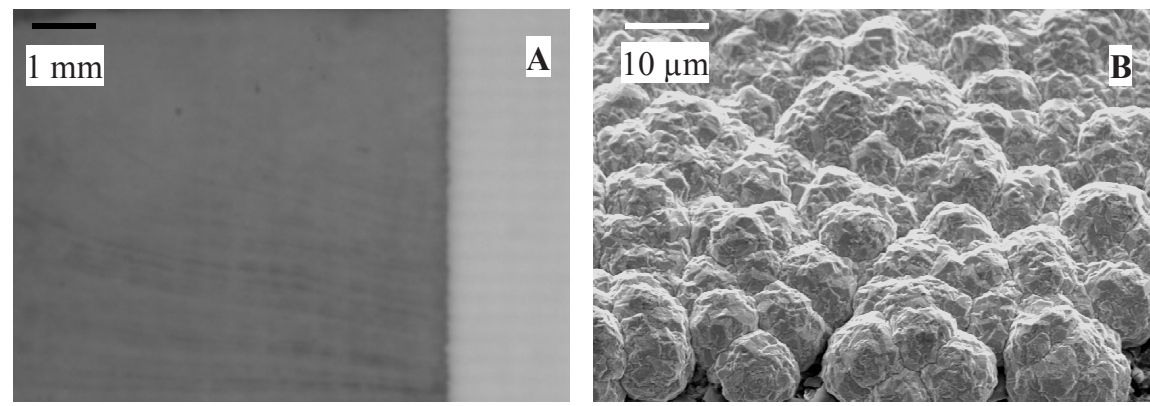

Figure 4.15: Topography of an inkjet printed-electroless plated structure on a plasma treated RO4003 substrate: (A) macroscopic and (B) microscopic.

\subsection{Conclusions}

Seed layers inkjet printed on untreated substrates could not be plated as silver delaminated during the plating process. The influence of plasma etching, deployed to modify the surface morphology of the substrates prior to printing, was studied with the aid of DoE. It was found that the flowrates of plasma producing gases are very important in determining the surface energy and surface roughness of the substrate material.

Of the 31 substrates yielded by the DoE, 2 were found to be suitable with respect to their improved adhesion to inkjet printed silver-electroless plated copper structures as well as to their post-treatment surface topography that is conducive to high-frequency RF applications. The magnitude of plasma treatment parameters for both these substrates were on the lower side of the chosen range of values i.e. equal to or less than level 0 . The coded and actual values of these 2 parameter sets are shown in table 4.5. They were only marginally rougher than an untreated substrate; the surface energy increase was also moderate. However, these proved to be sufficient, as the inkjet printed 
structures on these substrates were successfully copper plated using the electroless plating process. Scotch tape tests done on printed-plated structures on the selected substrates showed that the structures remained intact and there was no cohesive or adhesive failure.

Table 4.5: Coded and actual values of the selected parameter sets.

\begin{tabular}{|c|c|c|c|c|}
\hline Substrate $\rightarrow$ & \multicolumn{2}{|c|}{1} & \multicolumn{2}{c|}{19} \\
\hline Parameter $\downarrow$ & Coded & Actual & Coded & Actual \\
\hline P (watt) & -1 & 2900 & 0 & 3300 \\
t (min) & -1 & 20 & -2 & 10 \\
f_O $\mathbf{O}_{\mathbf{2}(\mathbf{m l} / \mathbf{m i n})}$ & -1 & 500 & 0 & 1000 \\
f_CF $_{4}$ & -1 & 50 & 0 & 100 \\
\hline
\end{tabular}

Hence, it was proved that $\mathrm{CF}_{4} / \mathrm{O}_{2}$ plasma etching is indeed an effective way of improving adhesion between the selected substrate material and the ink deposited on it. For applications (e.g. low frequency electronic applications) in which rougher substrates or those subjected to substantial etching do not alter the functional characteristics, substrates such as those highlighted in table 4.4 can be made use of, in order to utilise their enhanced mechanical interlocking with the deposited ink.

It needs to be mentioned that the DoE model does have some shortcomings, not the least of which is the omission of process pressure as a DoE parameter. Moreover, the selected parameters for DoE analysis and the model outcomes are highly case-specific; different substrate materials with different compositions require an independent selection and analysis of process parameters.

\subsection{References}

[1] T.H.J. Van Osch, J. Perelaer, A.W.M. de Laat and U.S. Schubert: "Inkjet printing of narrow conductive tracks on untreated polymeric substrates", Advanced Materials, Vol. 20, No. 2, p. 343, 2008.

[2] M. Mantysalo and P. Mansikkamaki: “An inkjet-deposited antenna for $2.4 \mathrm{GHz}$ applications", International Journal of Electronics and Communications, Vol. 63, No. 1, p. $31,2009$. 
Chapter 4

[3] J. Perelaer: "Microstructures prepared via inkjet printing and embossing techniques", Ph.D. Thesis - Eindhoven University of Technology, Eindhoven, the Netherlands, 2009.

[4] J.J.P. Valeton, K. Hermans, C.W.M. Bastiaansen, D.J. Broer, J. Perelaer, U.S. Schubert, G.P. Crawford and P.J. Smith: "Room temperature preparation of conductive silver features using spin-coating and inkjet printing", Journal of Materials Chemistry, Vol. 20, No. 3, p. 543, 2010.

[5] C.-Y. Kao and K.-S. Chou: "Electroless copper plating onto printed lines of nanosized silver seeds", Electrochemical and Solid-State Letters, Vol. 10, No. 3, p. D32, 2007.

[6] M.J.T.M. Van Bellegem, H.A. Ankersmit, R. Van Langh and W. Wei: "Solder on silver: historical usage and the problem of fretting”, Proceedings of Metal 2004 National Museum of Australia Canberra ACT, p. 104

[7] V. Subramanian, S.K. Volkman and D.R. Redinger: "Printed zinc oxide based electronics: materials, devices, and outlook", Proceedings of the Large-area, Organic and Printed Electronics Convention, Frankfurt, Germany, 2009.

[8] H.-Y. Lee and J. Qu: "Microstructure, adhesion strength and failure path at a polymer/roughened metal interface”, Journal of Adhesion Science and Technology, Vol. 17, No. 2, p. 195, 2003.

[9] R. Van Tijum and J.Th.M. De Hosson: "Effects of self-affine surface roughness on the adhesion of metal-polymer interfaces", Journal of Materials Science, Vol. 40, No. 13, p. 3503, 2005.

[10] S.-C. Park, S.-H. Cho, H.-C. Jung, J.-W. Joung and Y.-B. Park: "A study on improvement of interfacial adhesion energy of inkjet-printed $\mathrm{Ag}$ thin film on polyimide by $\mathrm{CF}_{4}$ plasma treatment", Korean Journal of Materials Research, Vol. 17, No. 4, p. $215,2007$.

[11] E.K. Chong, M.G. Stevens and K.E. Nissen: "Effect of surface roughness on the adhesion of electrolessly plated platinum to poly(ethylene terephthalate) films", The Journal of Adhesion, Vol. 79, No. 7, p. 667, 2003.

[12] J. Ge, R. Tuominen and J.K. Kivilahti: “Adhesion of electrolessly-deposited copper to photosensitive epoxy", Journal of Adhesion Science and Technology, Vol. 15, No. 10, p. $1133,2001$. 
[13] A. Schirmeisen, D. Weiner and H. Fuchs: "Measurements of metal-polymer adhesion properties with dynamic force spectroscopy", Surface Science, Vol. 545, p. 155, 2003.

[14] L.J. Martin and C.P. Wong: "Chemical and mechanical adhesion mechanisms of sputter-deposited metal on epoxy dielectric for high density interconnect printed circuit boards", IEEE Transactions on Components and Packaging Technologies, Vol. 24, No. 3, p. 416, 2001.

[15] Y. Nakamura, Y. Suzuki and Y. Watanabe: "Effect of oxygen plasma etching on adhesion between polyimide films and metal", Thin Solid Films, Vol. 290-291, p. 367, 1996.

[16] B.-I. Noh, C.-S. Seok, W.-C. Moon and S.-B. Jung: "Effect of plasma treatment on adhesion characteristics at interfaces between underfill and substrate", International Journal of Adhesion \& Adhesives, Vol. 27, No. 3, p. 200, 2007.

[17] K. Narushima and H. Ikeji: "Plasma surface modification of poly(aryl ether ether ketone) and surface metallization using copper metal”, Sen'i Gakkaishi, Vol. 65, No. 5, p. $127,2009$.

[18] S.H. Kim, S.W. Na, N.-E. Lee, Y.W. Nam and Y.-H. Kim: "Effect of surface roughness on the adhesion properties of $\mathrm{Cu} / \mathrm{Cr}$ films on polyimide substrate treated by inductively coupled oxygen plasma", Surface \& Coating Technology, Vol. 200, No. 7, p. 2072, 2005.

[19] Enthone Envision EC2130 Datasheet, Enthone Inc., USA.

[20] D.P. Obeng, S. Morrell and T.J. Napier-Munn: “Application of central composite rotatable design to modelling the effect of some operating variables on the performance of the three-product cyclone", International Journal of Mineral Processing, Vol. 76, No. 3, p. 181, 2005.

[21] K. Acikalin, F. Karaca and E. Bolat: "Central composite rotatable design for liquefaction of pine barks”, Fuel Processing Technology, Vol. 87, No. 1, p. 17, 2005.

[22] B. Manohar and S. Divakar: "Application of central composite rotatable design to lipase catalysed synthesis of m-cresyl acetate", World Journal of Microbiology \& Biotechnology, Vol. 18, No. 8, p. 745, 2002.

[23] D. Hegemann, H. Brunner and C. Oehr: "Plasma treatment of polymers for surface and adhesion improvement", Nuclear Instruments and Methods in Physics Research B, Vol. 208, p. 281, 2003. 
Chapter 4

[24] S.R. Cain, L.J. Matienzo and D.W. Wang: "Evaluation of a silicon-containing benzocyclobutene thermoset resin as a plasma etch stop", Journal of Materials Research, Vol. 5, No. 8, p. 1733, 1990.

[25] OCA20 Web Help, OCA video-based contact meter, DataPhysics Instruments $\mathrm{GmbH}$, Germany.

[26] A. Pizzi and K.L. Mittal: "Handbook of adhesive technology", Marcel Dekker Inc., ISBN: 9780824709860 .

[27] S. Sapieha, A.M. Wrobel and M.R. Werthheimer: "Plasma-assisted etching of paper", Plasma Chemistry and Plasma Processing, Vol. 8, No. 3, p. 331, 1988.

[28] StatGuide, Minitab 15 software program, Minitab Inc., USA.

[29] R. Waterman: Statistics Department, University of Pennsylvania, http://wwwstat.wharton.upenn.edu/ waterman/Teaching/701f99/Class04/Notes/node4.htm (accessed: February 2010). 


\section{Quantification of adhesion in an inkjet printed metal-polymer system}

In this chapter, adhesion between inkjet printed silver structures and a PCB material is quantified in terms of interfacial fracture energy. The pull-off test method, discussed briefly in chapter 3, was used to measure the tensile force required to cause fracture in the abovementioned metal-polymer system. The interfacial fracture energy was calculated from the measured tensile force.

To determine the influence of mechanical interlocking on interfacial adhesion, the PCB substrates were roughened to impart surface roughness of varying magnitude. Subsequently, silver structures were inkjet printed on these substrates. Additionally, polished substrates with minimal surface roughness, and hence negligible mechanical interlocking, were prepared and silver structures were inkjet printed on them as well. By comparing the interfacial fracture energy of these specimens, conclusions concerning the importance of mechanical interlocking are drawn in this chapter.

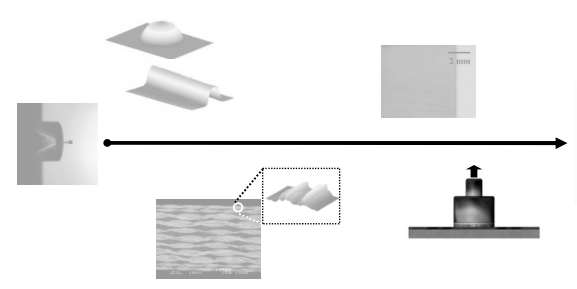


Chapter 5

\subsection{Introduction}

Reliability and robustness are crucial factors that determine the extent to which a process or a product is successful - inkjet printed electronics is no exception to that Structures fabricated by inkjet printing for electronics applications can be classified as thin films due to their high surface-to-volume ratio, as well as due to their micrometre/sub-micrometre thickness. In general, thin films are more vulnerable to thermo-mechanical stresses than the bulk material. Since the interface between a thin film and the substrate on which it is deposited is the most probable crack formation and propagation location, reliability and robustness of these films are strongly dependant on interfacial adhesion [1,2]. According to Mittal [3], 'adhesion is very important in thin film technology because the thin films are so fragile that these must be supported by more substantial substrates and the degree to which the film can share the strength of the substrate depends upon the adhesion between the two.' However, it is not easy to experimentally determine the interfacial strength of thin films because of their thinness. Although a number of methods have been proposed and tried out to measure the adhesion strength of thin films, most of them differ from each other not just with respect to the testing methodology itself, but also in terms of the experimental quantities measured as representations of adhesion. For instance, the pull-off test method gives the tensile force required to cause fracture of an interface as the output; on the other hand, the peel test method provides adhesion values in terms of energy or work done per unit area.

Of late, important results concerning the reliability of inkjet printed structures have been published. Caglar et al [4] discussed the mechanical reliability of inkjet printed silver structures in terms of tensile adhesive performance, determined using the pull-off test method. They tested the adhesion of silver structures to different flexible substrates, and concluded that in order to select the right substrate and ink combination for various electronics applications, adhesion performance is an important factor that needs to be taken into consideration. A few other publications, like the one by Kaydanova et al [5], have dealt with adhesion performance of inkjet printed structures using qualitative test methods like the scotch tape test. However, the effect of surface roughness and surface energy of the substrate material on the mechanical reliability of inkjet printed structures 
has not been given sufficient attention so far, though there are some exceptions like the work of Joo and Baldwin [6] and Park et al [7]. The former concluded that roughening the substrate does enhance the interfacial adhesion, based on experiments using a new adhesion test method called the modified button shear test (MBST) to estimate the interfacial fracture energy of NPS films. Park et al concluded based on $180^{\circ}$ peel testing that by plasma treating the polyimide substrate prior to inkjet printing, the interfacial debonding energy of the silver-polyimide system can be increased markedly.

It would be fair to assume that extensive investigations on the mechanical reliability of inkjet printed structures would be undertaken in the near future, owing to the increasing number of research initiatives focussing on inkjet printing as a tool to deposit functional materials. Herein arises a question as to whether it is possible to draw conclusions concerning the mechanical reliability of inkjet printed structures from the experimental results of thin film testing available from comparable research areas. Physical vapour deposition (PVD) and sputter coating are two such research areas that immediately seem to be of interest. Therein lies a problem: adhesion of thin films is affected by the mode of deposition, the rate of deposition, the film thickness, the type of substrate, the substrate temperature, the process pressure etc [3]. Hence, any test procedure used to determine adhesion of inkjet printed thin films, and thus their mechanical reliability, should be developed/optimised keeping the nature of the deposited films in mind.

Chemical bonding and mechanical interlocking are the two most important mechanisms of adhesion in a metal-polymer system [6,8]. The other bonding mechanisms, namely electrostatic forces, diffusion and physical adsorption can be neglected for the system under study [6]. The main objective of this study is to segregate the two important components of adhesion, namely chemical bonding and mechanical interlocking, so as to quantitatively prove their significance to the reliability and robustness of the end products.

Throughout this research, the Scotch tape test method was used to qualitatively test inkjet printed structures for adhesion. In this chapter, a quantitative approach in the form of the pull-off test method is presented. 
Chapter 5

\subsection{Important mechanisms of metal-polymer adhesion}

Mechanical interlocking and chemical bonding are considered to be the most important adhesion mechanisms in a metal-polymer system. These two mechanisms are explained in this section.

\subsubsection{Mechanical interlocking}

Strictly speaking, mechanical interlocking is not an intrinsic adhesion mechanism, and is not a phenomenon at the molecular level. It refers to the anchoring of one entity by another, by virtue of their surface roughness. In the context of this study, mechanical interlocking refers to the anchoring of the inkjet printed silver structure by the polymeric substrate. Due to the anchoring, additional energy should be supplied to separate the silver structure and the substrate.

The adhesion enhancement due to surface roughness is associated with a mechanism that prevents the separation of the interface from occurring completely along the true interface [9]. In the presence of a propagating delamination at the interface, interpenetrating features de-bond to deflect the crack front in directions that reduce its propensity to propagate, leading to greater energy requirement to separate the adhering entities [10]. Therefore, the interface becomes more robust i.e. tougher by virtue of mechanical interlocking.

In some cases however, the increased roughness can lead to decreased adhesion strength. This can happen when the deposited silver fails to penetrate the roughness valleys, which causes voids between the silver and the substrate. This allows the accumulation of moisture, which undermines the strength of the interface [9].

\subsubsection{Chemical bonding}

The chemical bonding refers to the formation of covalent bonds at the interface when the silver ink comes into contact with the polymeric substrate. This usually occurs as a result of charge transfer from silver to the substrate. The reaction involves hydrophilic 
groups at the surface of the polymeric substrate, which readily accept the charge from silver. This transfer results in the formation of a charge transfer complex, which enhances the interfacial adhesion [9].

Generally, polymers are not very conducive to chemical bond formation. It is for this reason that surface modification methodologies like plasma treatment are used. Such surface modification techniques impart functional i.e. hydrophilic groups on the surface of the substrate.

\subsection{The pull-off test method}

The pull-off test method was already described briefly in chapter 3 . Since it is the crux of this chapter, it is given a detailed treatment here.

The test specimen consists of a film and a substrate on which the film is deposited. In the present context, the film is an inkjet printed silver structure, and the substrate, a PCB material. An aluminium dolly is glued to the specimen to be tested, and is subsequently pulled-off by applying a vertical load. In order to get meaningful results concerning the adhesion strength of the specimen from this test method, the selection of the glue is crucial. Its adhesive properties should be such that the dolly-glue adhesion as well as the specimen-glue adhesion is stronger than either the cohesive strength of the printed silver layer or the adhesive strength of the silver-substrate interface. In addition to that, the cohesive strength of the glue should also be greater than either the cohesive strength of the printed silver layer or the adhesive strength of the silver-substrate interface. The pull-off force required to fracture the weakest interface/component is the outcome of this test. The type of failure can be cohesive, adhesive or cohesive-adhesive. The averaged critical tensile stress at fracture $\left(\sigma_{c}\right)$ is calculated using:

$$
\sigma_{c}=F_{p u l l} / A_{\text {dolly }}
$$

Here, $F_{\text {pull }}$ is the pull-off force, and $A_{\text {dolly }}$, the cross-section of the base of the dolly that is glued to the specimen. 
Chapter 5

The pull-off test method has certain drawbacks associated with it, the most important of which are listed below:

- Simple tensile tests are difficult to perform, as most of the times they involve a complex interaction of tensile and shear forces, making the interpretation of the test results difficult [3].

- This test methodology usually results in large plasticity in the test area, especially at the edge; hence, it is not easy to correlate the pull-off force to the work of adhesion [11].

- The stress concentration at the edges leads to low pull-off force values, resulting in lower calculated $\sigma_{c}$ values. In other words, the test outcome is geometrydependant.

Despite these drawbacks, the pull-off test method was nevertheless selected, as it is a straightforward method to compare the adhesion of specimens under consistent conditions [11]. Moreover, the other available test methods, such as the peel test method, also have certain disadvantages, as discussed by Mittal [3]. For instance, he pointed out that in order to make any useful measurement, the film must be removed from the substrate completely, which limits the applicability of this technique to those interfaces that exhibit relatively poor adhesion.

The pull-off test method can be executed with two different configurations namely matched and unmatched. While the former refers to the case in which the silver structure and the dolly are matched (figure 5.1(A)), the latter refers to the case in which the silver structure covers a larger area than the dolly, and hence they are unmatched (figure 5.1(B)).

In this study, the 'matched' configuration was adopted. The reason is a practical one: it was observed from initial experimental trials that when the dolly is glued onto the specimen, the epoxy glue is squeezed out of the interface and accumulates along the circumference of the test area. Even after wiping the excess glue off, it was found after the curing of the glue that a thin layer of glue still remains along the periphery of the test area. This results in an undesired increase in the pull-off area, and could also lead to high local stress concentrations. Hence, isolation of the test area using a cutting tool was 
unavoidable. This process is explained in the next section. It was also decided to select an equipment that clamps the specimen by applying a normal load adjacent to (and surrounding) the silver structure, so as to minimise the bending of the substrate.
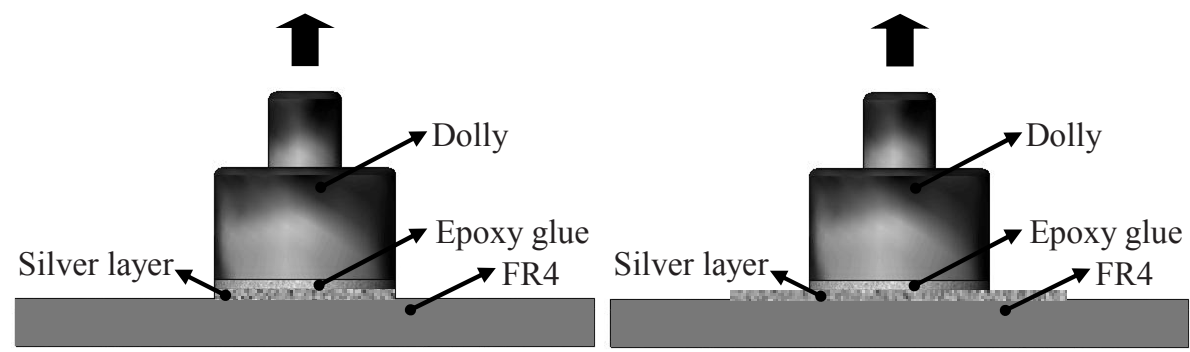

Figure 5.1: The two variants of the pull-off test method: $(A)$ matched and $(B)$ unmatched. The arrows indicate the direction of pull-off.

\subsection{Experiments}

\subsubsection{Materials and methods}

The materials and equipments used for this study are listed in table 5.1:

Table 5.1: Details of the important raw materials and equipments relevant to this chapter.

\begin{tabular}{|l|l|l|}
\hline Raw material/equipment & Type/Name & Supplier \\
\hline Substrate & FR4 & Park Electrochemical Corp., \\
Inkjet ink & $\begin{array}{l}\text { Urganic silver complex } \\
\text { compound (TEC-IJ-040) }\end{array}$ & InkTec Company Limited, \\
Etchant to roughen copper foil & AlphaPREP & Enthone Inc., USA \\
Interferometer & Micromap 560 & ATOS GmbH, Germany \\
Surface roughness measurement & DEKTAK surface & Veeco Instruments Inc., USA \\
Pull-off adhesion tester & profiler & DeFelsko Corp., USA \\
Epoxy adhesive for pull-off testing & Araldite 2011 & Huntsman Advanced Materials, \\
\hline
\end{tabular}


Chapter 5

Table 5.2 lists the properties of the materials used in this study; these properties were used to calculate the interfacial fracture energy.

Table 5.2: Material properties.

\begin{tabular}{|l|l|l|l|}
\hline Material & Function & $\begin{array}{l}\text { Young's } \\
\text { modulus (GPa) }\end{array}$ & $\begin{array}{l}\text { Poisson's } \\
\text { ratio }\end{array}$ \\
\hline Aluminium & Dolly & 71 & 0.3 \\
Epoxy & Glue & 3.2 & 0.4 \\
Silver & Measurand & 83 & 0.37 \\
Polyester & Flexible substrate & 3 & 0.4 \\
FR4 & Rigid substrate & 18.6 & 0.25 \\
\hline
\end{tabular}

RO4003 substrate, used for the DoE-based plasma treatment described in the previous chapter, was not selected for this study. The reason being, the tailor-made RO4003 substrates procured from the supplier (Rogers Corporation, USA) specifically for inkjet printing exhibited poor resistance to tensile stresses induced by the pull-off test. The interfacial bond strength between the individual laminates that make up the substrate was found to be lower than the adhesive strength between the silver structure and the substrate. This can be seen clearly in figure 5.2, which shows inter-ply failure of the substrate. This observation is, however, holds true only for the tailor-made substrates procured for inkjet printing, and not necessarily for the RO4003 substrates that are commercially available.
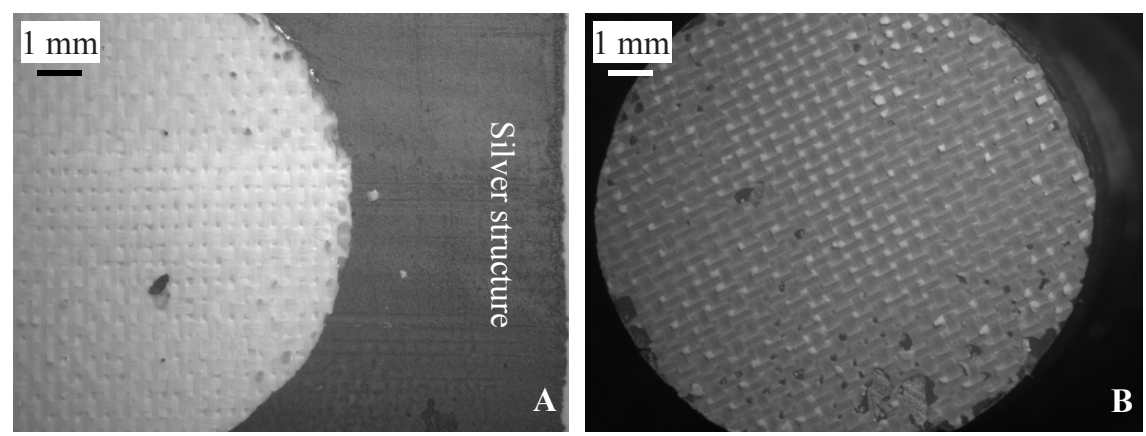

Figure 5.2: The interlaminate failure of RO4003 substrate during pull-off testing; (A) the delaminated substrate, and $(B)$ the underside of the dolly showing the top-most ply pulled off the substrate. 


\subsubsection{Specimen preparation}

As mentioned before, the main objective of this study is to segregate the adhesion components resulting from mechanical interlocking and chemical bonding. In addition to that, it was intended to determine the influence of the magnitude of surface roughness on adhesion. To accomplish this, the following approach was adopted:

1. Imparting varying levels of surface roughness on a set of FR4 substrates.

2. Preparing another set of FR4 substrates in such a way that their surface is as smooth as possible; this will eliminate the influence of mechanical interlocking on adhesion to a large extent.

3. Inkjet printing of silver test structures on all the substrates.

4. Pull-off adhesion testing of these test structures and recording the critical tensile stress at fracture.

5. Calculating the interfacial fracture energy using the critical tensile stress, for all the measurements.

6. Segregating the contributions of mechanical interlocking and chemical bonding to adhesion; this can be done by comparing the difference in interfacial fracture energy for polished and roughened substrates.

Plasma treatment, which was discussed elaborately in chapter 4, was not used for roughening the substrates in this study. The reason being, in addition to roughening the surface of the substrate, plasma also modifies it chemically, making the segregation of adhesion components impossible. Hence, a different methodology based on a pressing process was adopted for this purpose. Figure 5.3 illustrates the step-by-step procedure leading to roughened substrate surface. 


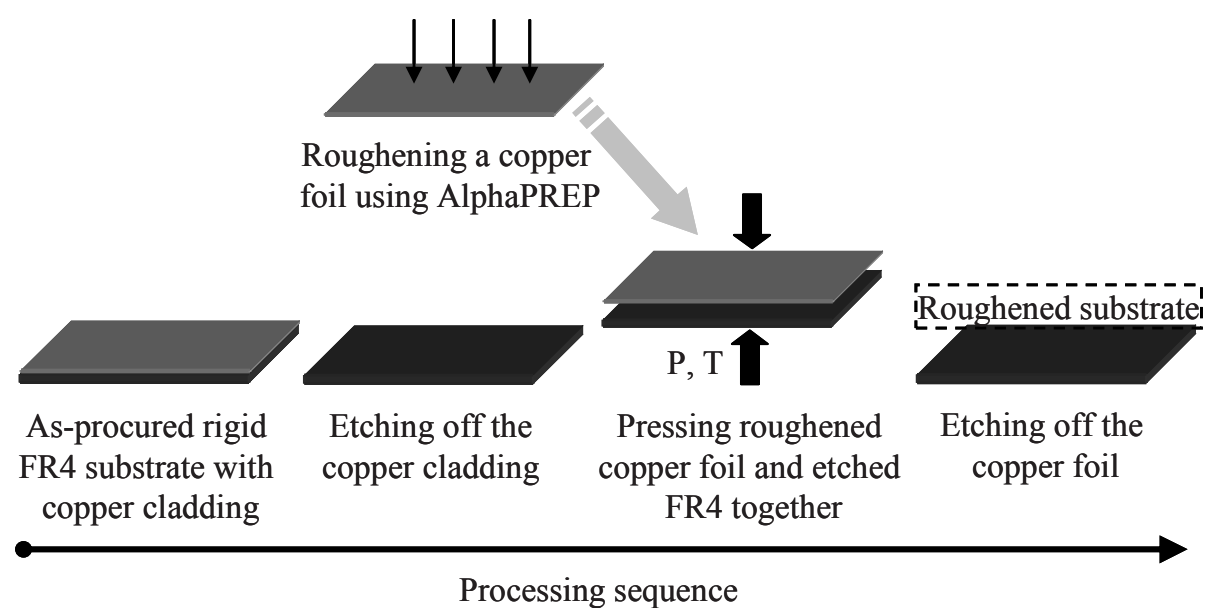

Figure 5.3: Schematic of the methodology deployed to roughen the substrates prior to inkjet printing.

The as-procured FR4 substrates had an inherent copper cladding. As the first step, the cladding was etched away chemically using the routine PCB wet etching process. Thin, smooth copper foils were subsequently roughened using AlphaPREP organo-metallic conversion coating. This process is based on a peroxide-sulphuric chemistry, which etches away minimal amounts of copper [12]. By passing different copper foils through the automated etching equipment at different feed rates, surface roughness of varying magnitudes was imparted on them. Seven copper foils with different surface roughness values were prepared and the roughness values were measured. Subsequently, each copper foil was pressed onto different FR4 substrates. During the pressing process, the platens of the press were maintained at $182^{\circ} \mathrm{C}$; the pressure applied on the copper foilFR4 layers was 23 bars, and the duration of the pressing process was 100 minutes. These parameters, determined based on prior experience at Thales Nederland B.V., were sufficient to ensure that the polymer (epoxy) matrix of FR4 deformed around the roughness peaks of the copper foil, as the former's glass transition temperature $\left(T_{g}\right)$ was exceeded. Upon curing, the copper foil was chemically etched off the FR4 substrates. This left behind the negative impression of the surface topography of copper foils on FR4 substrates. The surface roughness of these FR4 substrates was measured again, and the values were found to be almost the same as that of the corresponding copper foils. Each substrate was subjected to three roughness measurements at different spots. Then, each substrate was cut into three pieces. 
The methodology followed here is convoluted, involving many steps, which is not inline with the aim of this thesis; nevertheless, it was adopted for this study as the means were readily available. This however does not mean that this methodology will form a part of the proposed process chain. As mentioned before, the sole purpose of using this pressing-based methodology was to impart varying levels of roughness on the substrate prior to inkjet printing. It should be mentioned here that the surface morphologies of the substrates prepared by this method were more printing-friendly than the surface morphologies of the substrates discussed in chapter 3 . This is owing to the fact that the former did not have surface porosity.

To prepare substrates that have minimal surface roughness, polishing was used. Firstly, two FR4 substrates were hand-polished using $1 \mu \mathrm{m}$ diamond abrasives sprayed on a Struers DP-Mol polishing cloth mounted on a disc, for 3 minutes. Subsequently, another polishing cloth of the same type loaded with $0.25 \mu \mathrm{m}$ diamond abrasives was used to fine-polish the substrates, the duration being 5 minutes. The rotational speed of the polishing disc was maintained at 200 RPM. After polishing, the FR4 substrates were inspected using an interferometer. It was found that the average roughness was about $100 \mathrm{~nm}$, which indicates a drastic reduction in roughness compared to the substrates prepared by pressing. Both the polished FR4 substrates were subjected to interferometer measurements at three different spots.

Silver test structures measuring $25 \mathrm{~mm} \times 25 \mathrm{~mm}$ were inkjet printed all the prepared substrates. The printed structures were sintered on a table heater for 30 minutes at $150^{\circ} \mathrm{C}$.

Henceforth in this chapter, the printed structure-substrate combination is referred as specimen.

\subsubsection{Adhesion testing}

The eight sets of specimens (seven roughened and one polished) were subjected to pulloff testing following the procedure already described. Aluminium dollies were glued on the specimens. To ensure that only a thin, uniform layer of glue is present between the 
Chapter 5

dolly and the specimen, a compressive load was applied on the dolly for 10 seconds. Excess glue around the test area was wiped off using a cloth. After the glue was completely cured, the test area was isolated using a cutting tool. To apply a tensile load on the dolly, a portable pull-off tester was used. The tester and its accessories are shown in figure 5.4. An additional specimen was prepared to find out the thickness of the glue layer using SEM. This detail was needed for calculations described later in this section.

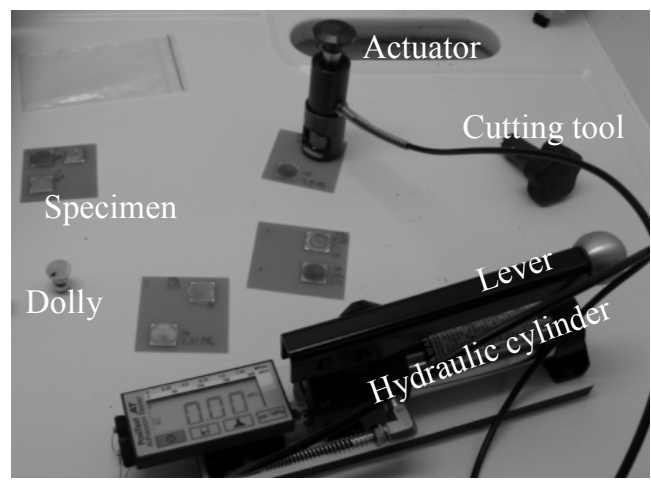

Figure 5.4: The pull-off adhesion testing equipment and accessories.

The dolly is held in place by a quick-coupling mechanism that is part of the actuator. The load is applied on the dolly via the actuator, which is in turn activated by a handoperated hydraulic cylinder. The actuator and the quick-coupling mechanism are connected in such a way that the load is applied vertically. All the pull-off tests were performed at a fairly constant rate of load application $\left(\sim 0.5 \mathrm{MPa}^{-1} \mathrm{~s}^{-1}\right)$, to ensure consistency. The rate is indicated live on the graphical pull-rate indicator on the digital display. When the critical tensile stress sufficient to cause fracture is reached, the dolly is lifted (pulled) off the specimen. The critical tensile stress at fracture, indicated on the digital display, was recorded for all the tested specimens.

The pulled off dollies were studied under an optical microscope as well as a SEM, to ascertain the nature of fracture i.e. whether the fracture was adhesive, cohesive or cohesive-adhesive. The recorded critical stress values were used to calculate the interfacial fracture energy according to the formula proposed by Kendall [13], and subsequently modified by Joo et al [6]: 


$$
\frac{F_{\text {pull }}}{\pi a^{2}}=\sigma_{c}=\sqrt{\frac{2 G_{a}}{\sum_{i=1}^{n} \frac{t_{i}}{K_{i}}}}
$$

where, $F_{\text {pull }}$ is the pull-off force $(\mathrm{N}), \sigma_{c}$ is the critical stress that causes fracture $\left(\mathrm{N} . \mathrm{m}^{-2}\right)$, $a$ is the contact radius (m), $G_{a}$ is the interfacial fracture energy at the fracture interface $\left(\mathrm{J} \cdot \mathrm{m}^{-2}\right), t$ is the thickness of each elastic layer $(\mathrm{m}), n$ is the number of elastic layers, and $K$ is the bulk modulus, a function of Young's modulus $E\left(\mathrm{~N} . \mathrm{m}^{-2}\right)$ and Poisson's ratio $v$ of each elastic layer; it is defined as:

$$
K=\frac{E}{3(1-2 v)}
$$

Originally, eqn. 5.2 was proposed by Kendall in the context of determination of interfacial fracture energy when an elastic material bonded to two rigid objects (an infinite rigid plane i.e. substrate and a rigid disc i.e. dolly); the modification by Joo et al was done to include multiple elastic materials involved in pull-off testing. It should be mentioned that this formula neglects the effects of plasticity at the test area, as well as crack branching.

\subsection{Results and discussion}

The roughness values of the FR4 substrates prepared using the methodology illustrated in figure 5.3, are shown in table 5.3. These roughness values are reasonable for inkjet printing, as most of them are approximately of the same magnitude as that of the substrates that were selected from the DoE described in chapter 4. Substrate number ' 0 ' stands for the polished one. 
Chapter 5

Table 5.3: Surface roughness values of the FR4 substrates prepared for pull-off testing.

\begin{tabular}{|l|l|}
\hline Substrate nr. & $\begin{array}{l}\text { Roughness } \\
\left(\mathbf{R}_{\mathbf{a}}\right)(\boldsymbol{\mu m})\end{array}$ \\
\hline 0 & 0.10 \\
1 & 0.51 \\
2 & 0.68 \\
3 & 0.70 \\
4 & 0.89 \\
5 & 1.05 \\
6 & 1.08 \\
7 & 1.16 \\
\hline
\end{tabular}

The results of the pull-off tests, in terms of critical tensile stress at fracture, are shown in figure 5.5. The thickness of the silver structure, the glue layer and FR4 substrate were determined to be $0.8 \mu \mathrm{m}, 110 \mu \mathrm{m}$ and $1.6 \mathrm{~mm}$, respectively. The interfacial fracture energy values, calculated based on the critical tensile stress values, are shown in figure 5.6. The tensile strength values of the inkjet printed silver-FR4 interface are consistent with the literature $[4,8,14]$. The increase in critical tensile stress due to substrate roughening, such as that seen in figure 5.5, was also observed by Ge et al for an electroless plated metal-polymer system [8]. However, the interfacial fracture energy values are way lower (by nearly two orders of magnitude) when compared to those experimentally determined by Park et al [7]. But Park et al used the peel test method, the mechanics of which are fundamentally different from those of the pull-off test method.

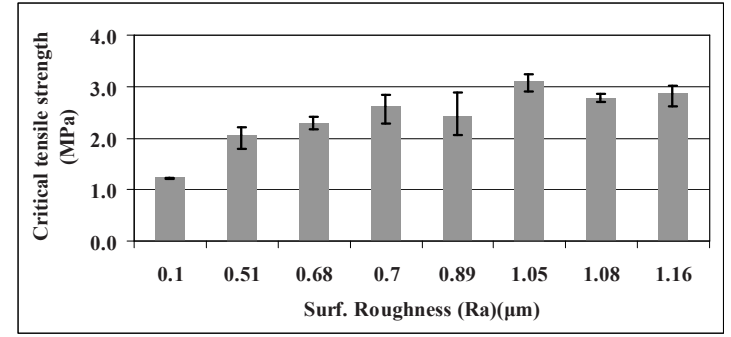

Figure 5.5: The pull-off test results depicting critical tensile stress values at fracture. 


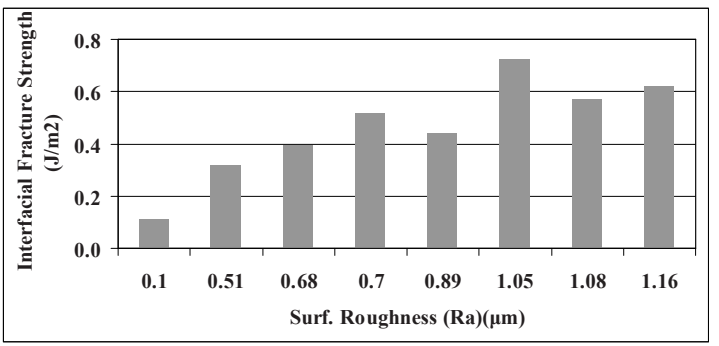

Figure 5.6: The interfacial fracture strengths calculated based on critical tensile stress values.

From figures 5.5 and 5.6, it can be directly concluded that mechanical interlocking promotes interfacial adhesion between inkjet printed silver and FR4. The interfacial fracture energy of the specimen prepared on the polished substrate (with an average roughness of $0.1 \mu \mathrm{m}$ ) is $0.11 \mathrm{Jm}^{-2}$, while that of the specimen prepared on the least roughened substrate (with an average roughness of $0.51 \mu \mathrm{m}$ ) is $0.32 \mathrm{Jm}^{-2}$. This is an increase of nearly $200 \%$, which is very high indeed. This is not an one-off occurrence; other specimens prepared on substrates with higher roughness values exhibited even higher interfacial fracture energies, the maximum being $0.72 \mathrm{Jm}^{-2}$. By-and-large within the current experimental domain, the interfacial fracture energy increases with an increase in surface roughness. This is understandable, as the mechanical anchors have to be broken, which requires additional energy. But the increase in the critical tensile stress needed for fracture is minimal for surface roughness values greater than $0.68 \mu \mathrm{m}$. This reveals the limitation in the cohesive/interfacial fracture toughness of this inkjet printed metal-polymeric substrate system.

The failure mode also shifts from adhesive for smooth (polished) substrate towards cohesive/cohesive-adhesive for roughened specimens. That is, in the case of the polished substrate, the silver structure was pulled almost clean off the substrate i.e. the failure mode was adhesive. For the roughened substrates, the failure occurred either within the silver structure (cohesive) or partially within the silver structure and partially at the silver-substrate interface (cohesive-adhesive). These three types of failures are shown in figure 5.7 . 

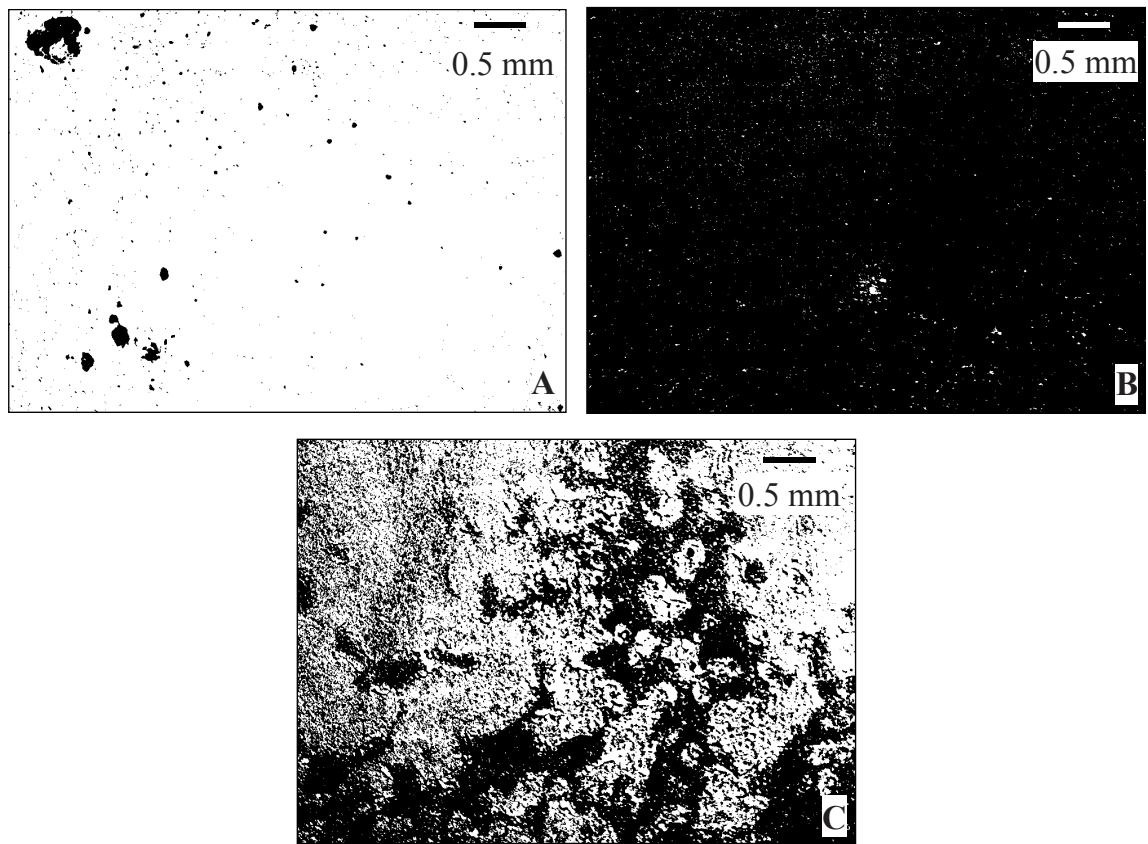

Figure 5.7: The three modes of fracture encountered during the pull-off tests, shown after image analysis: $(A)$ adhesive, $(B)$ cohesive and (3) cohesive-adhesive. The dark areas in these pictures indicate the left over silver on the substrate, which is represented by the bright areas.

The cohesive-adhesive failure was the most common type encountered during the pulloff tests in this study. This is consistent with the literature $[4,8]$ dealing with similar metal-polymer systems. It is however difficult to comment on the crack path for this type of test, as the material is pulled off the substrate in an instant. Unlike the peel test, where an initiated crack takes its course that is dependent on the path of least resistance, the pull-off test method does not have a readily definable crack path. The pull-off test can have multiple cracks initiated and propagated by the applied tensile load.

Since the polished specimens exhibited adhesive failure at the silver-FR4 interface, it is fair to assume that the contribution of mechanical interlocking towards the interfacial fracture energy is negligible. Hence, the calculated interfacial fracture energy of 0.11 $\mathrm{Jm}^{-2}$ can be considered as the result of chemical bonding between the two materials. It is however difficult to attribute the increased interfacial fracture energy of roughened substrates solely to mechanical interlocking. It is very likely that chemical bonding also 
played a role, as a result of an increase in interfacial area due to roughening. Figure 5.8 compares the surface roughness profiles of specimen number 1 and 5 listed in table 5.3. This figure clearly shows that the interfacial area of specimen number 5 is a lot higher than that of specimen number 1 .

Using image analysis, it was calculated that this increase in interfacial area due to higher surface roughness is three-fold. An increased interfacial (contact) area between the printed structure and the substrate leads to more bonding opportunities. That said, the influence of mechanical interlocking on interfacial fracture energy is as important as that of chemical bonding for the chosen metal-polymer substrate system and the metal deposition method. As shown in figure 5.8, the sidewalls of the roughness peaks and valleys are rough, giving rise to the anchoring mechanism. The cohesive-adhesive failure of most roughened specimens (and the cohesive failure in the remaining roughened specimen) point to the fact that anchoring led the crack to move away from the interface, thereby increasing the energy needed to cause fracture.

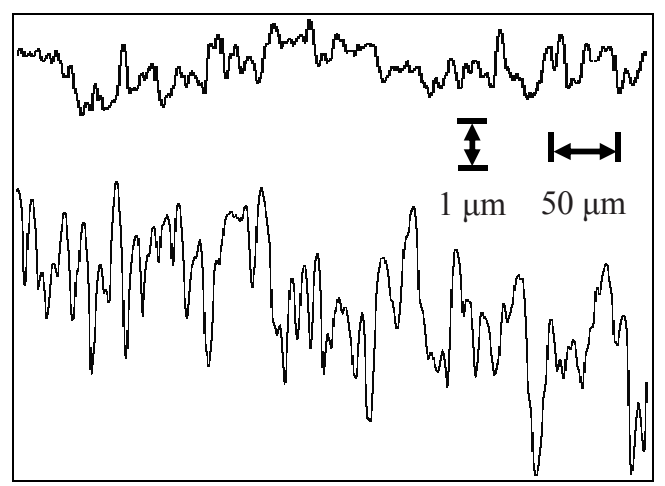

Figure 5.8: The surface roughness $\left(\mathrm{R}_{\mathrm{a}}\right)$ comparison of substrate number 1 and 5, as listed in table 5.3.

Figure 5.9 aids in a better visualisation of the proposed influence of the roughness profiles on mechanical interlocking, and consequently, adhesion. This figure shows the 3D interferometer images of substrate number 1 at different magnifications. It can be seen clearly that the roughness profiles are complex in nature, with multiple anchoring locations, even on a moderately roughened substrate. 
Chapter 5

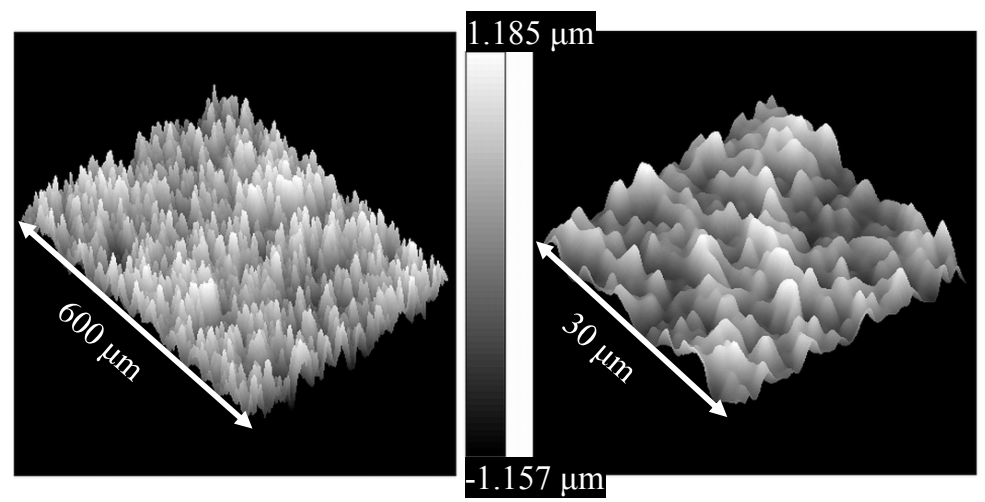

Figure 5.9: Interferometer images depicting the surface roughness $\left(\mathrm{S}_{\mathrm{a}}\right)$ of substrate number 1 at different magnifications.

\subsection{Conclusions}

A pressing-based methodology was adopted to roughen the surface of the substrates without increasing their surface energy, unlike the plasma treatment. The substrates roughened by this methodology were first inkjet printed upon, and then subjected to pull-off adhesion testing. The results of pull-off adhesion tests highlighted the importance of surface roughness of the substrate.

It can be concluded from the results of pull-off testing that the interfacial fracture energy of an inkjet printed silver-FR4 interface is highly enhanced by an increase in surface roughness. The contribution of mechanical interlocking to the interfacial fracture energy is as important as that of chemical bonding for the chosen materials and deposition technique. In the context of inkjet printing, the conclusion concerning the importance of mechanical interlocking can be extended to other rigid substrates as well, as long as the roughness scales are comparable. The reason being, mechanical interlocking is a material-independent phenomenon.

Since reliability and robustness of an inkjet printed structure is highly dependent on interfacial adhesion, roughening the substrate to reasonable surface roughness values is a good approach towards fabricating reliable and robust inkjet printed structures. It is however important to take the strength of the substrate into consideration, before any 
attempt to improve the interfacial adhesion between the printed structure and the substrate. As discussed in section 5.4 with the example of RO4003 substrate material, the interfacial strength within this substrate itself (i.e. the cohesion between the individual plies of the substrate) could be lower than the interfacial adhesion between the printed structure and the substrate. In this case, the reliability and robustness of the end product tends to have a greater dependence on the substrate itself, rather than the printed structure.

Based on experimental evidences presented in this chapter as well as in chapter 4, it is proposed that the reasonable roughness values are in the order of the layer thickness of a single inkjet printed layer.

\subsection{References}

[1] M. Omiya, K. Kishimoto, H. Inoue and M. Amigai: "Measurement of copper thin film adhesion by multi-stages peel test", Proceedings of the $4^{\text {th }}$ International Symposium on Electronic Material and Packaging, p. 472, 2002.

[2] H. Hirakata, T. Kitamura and T. Kusano: "Pre-cracking technique for fracture mechanics experiments along interface between thin film and substrate", Engineering Fracture Mechanics, Vol. 72, No. 12, p. 1892, 2005.

[3] K.L. Mittal: “Adhesion measurement of thin films", Electrocomponent Science and Technology, Vol. 3, p. 21, 1976.

[4] U. Caglar, K. Kaija and P. Mansikkamaki: "Evaluation of adhesion pull-off performance of nanoparticle-based inkjet-printed silver structure on various substrates", International Journal of Nanotechnology, Vol. 6, No. 7-8, p. 681, 2009.

[5] T. Kaydanova, A. Miedaner, J.D. Perkins, C. Curtis, J.L. Alleman and D.S. Ginley: "Direct-write inkjet printing for fabrication of barium strontium titanate-based tunable circuits", Thin Solid Films, Vol. 515, No. 7-8, p. 3820, 2007.

[6] S. Joo and D.F. Baldwin: "Interfacial adhesion of nano-particle silver interconnects for electronics packaging application", $58^{\text {th }}$ IEEE Electronic Components and Technology Conference p. 1417, 2008.

[7] S.-C. Park, S.-H. Cho, H.-C. Jung, J.-W. Joung and Y.-B. Park: "A study on improvement of interfacial adhesion energy of inkjet-printed Ag thin film on polyimide 
Chapter 5

by $\mathrm{CF}_{4}$ plasma treatment", Korean Journal of Materials Research, Vol. 17, No. 4, p. $215,2007$.

[8] J. Ge, R. Tuominen and J.K. Kivilahti: “Adhesion of electrolessly-deposited copper to photosensitive epoxy", Journal of Adhesion Science and Technology, Vol. 15, No. 10, p. 1133, 2001.

[9] J. Ge: "Interfacial adhesion in metal/polymer systems for electronics", Ph.D. Thesis

- Helsinki University of Technology, Espoo, Finland, 2003.

[10] M.P. Larsson and M.M. Ahmad: "Improved polymer-glass adhesion through micro-mechanical interlocking”, Journal of Micromechanics and Microengineering, Vol. 16, p. 161, 2006.

[11] O. Dos Santos Ferreira: "Adherence of nickel films to inorganic materials", Ph.D. Thesis - Eindhoven University of Technology, the Netherlands, 2006.

[12] AlphaPREP PC-7030 Datasheet, Enthone Inc., USA.

[13] K. Kendall: "The adhesion and surface energy of elastic solids", Journal of Physics D: Applied Physics, Vol. 4, p. 1186, 1971.

[14] M.P.K. Turunen, P. Marjamaki, M. Paajanen, J. Lahtinen and J.K. Kivilahti: "Pulloff test in the assessment of adhesion at printed wiring board metallisation/epoxy interface", Microelectronics Reliability, Vol. 44, p. 993, 2004. 


\title{
6. Fabrication of RF circuit structures using inkjet printing-electroless plating: a proof-of-concept
}

\begin{abstract}
A proof-of-concept of the methodology for fabrication of RF circuit structures, described in chapter 4 , is presented. The validation was done by fabricating an S-band filter and a transmission line on RO4003 substrate, and comparing their RF performance with that of similar RF circuit structures fabricated using the conventional lithography-based method. It was found that the performance of the inkjet printed-electroless plated circuit structures is comparable to that of the conventionally fabricated structures. The reproducibility of the proposed fabrication methodology was also verified with favourable results, thus proving its suitability for practical RF applications.
\end{abstract}


Chapter 6

\subsection{Introduction}

\subsubsection{An inkjet printing-based process chain}

It was illustrated in the previous chapters that due to the limitations posed by solderability, adhesion and substrate surface morphology, it is not straightforward to use inkjet printing as a one-step fabrication method for functional (RF-) electronics. In the context of this research, it can be concluded that it is not possible to fabricate robust conductive circuit structures using inkjet printing without any pre- and post-processing steps. Based on the results of chapter 4, a new process chain for inkjet printing-based fabrication of conductive circuit structures is proposed in figure 6.1. The process chain is depicted in this figure in the form of a 'Cause-and-Effect (or Ishikawa) Diagram'.

Adhesion improvement

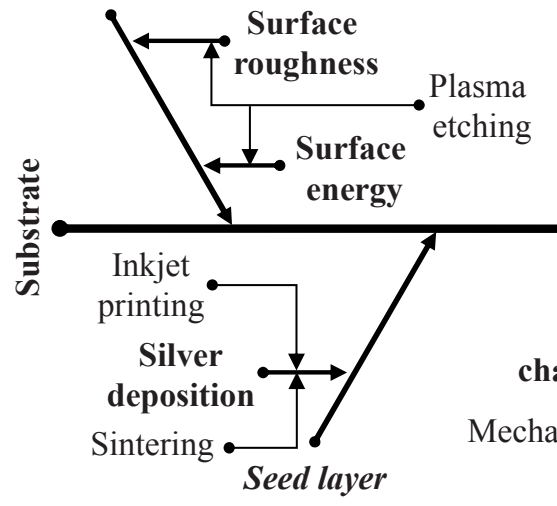

Thicker

tracks

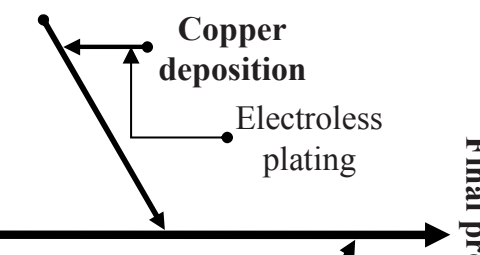

RF testing •-

Functional characterisation

Figure 6.1: Cause-and-effect diagram representing the components of the proposed process chain.

In this process chain, 4 major steps are involved in preparing the final product i.e. substrate with conductive circuit structures: improving the adhesion properties of the substrate, inkjet printing of the silver seed layer, electroless copper deposition and, finally, qualification by functional characterisation. Each of these steps contains substeps, as indicated in figure 6.1 .

Although the proposed process chain has more steps than what was desired at the beginning of this research, all the steps and sub-steps are vital to the realisation of the 
end product. In fact, if substrate materials with surface morphology favourable to the deposited ink can be procured from the supplier, it is then possible to leave out the plasma etching step from the proposed process chain. In any case, the reduction in the number of process steps when compared to the conventional lithography-based fabrication of conductive circuit structures on PCB is marked. This is evident from figure 6.2, which depicts the conventional process chain to fabricate conductive structures. The sub-steps constituting each step are not depicted in this figure.

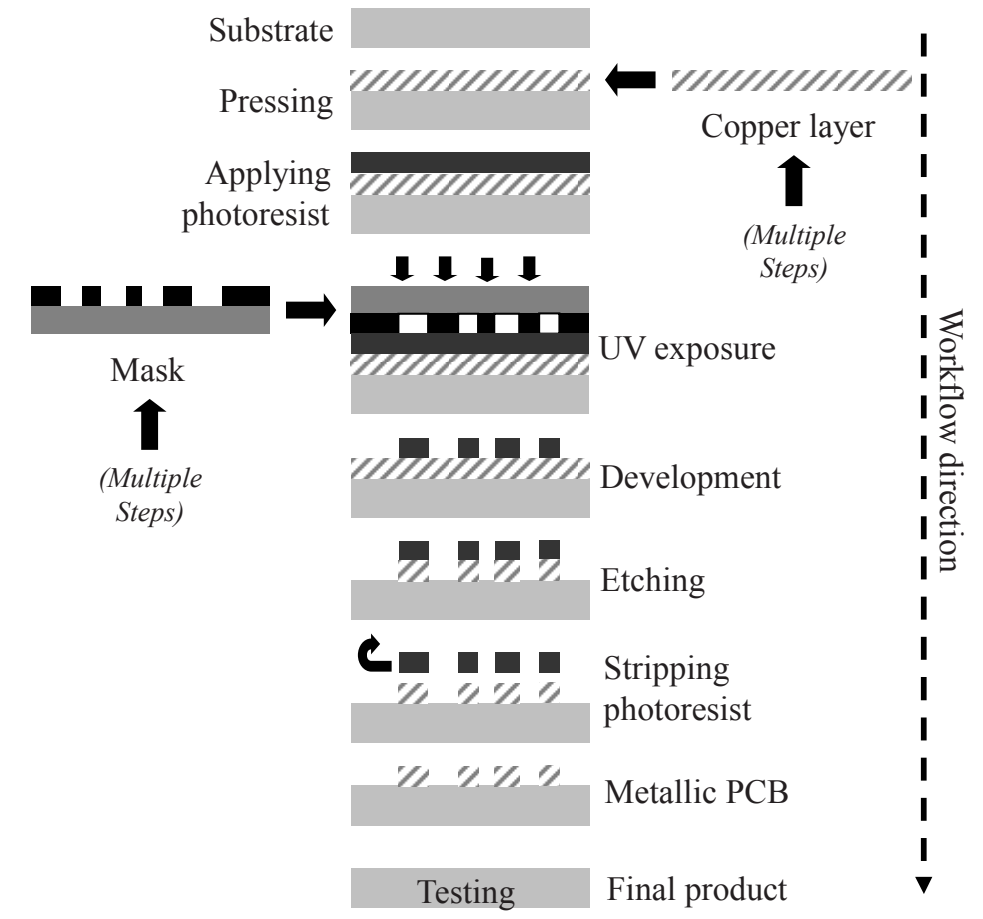

Figure 6.2: Steps involved in conventional lithography-based fabrication of conductive circuit structures [1].

\subsubsection{A proof-of-concept}

The proposed process chain has fewer steps when compared to the conventional process chain, as shown in the previous section. Moreover, the inkjet printing-based process chain minimises material wastage due to its additive nature. But before concluding that it is possible to replace the conventional method by the proposed one for specific 
Chapter 6

applications, a proof-of-concept is needed to validate the proposed process chain. For this purpose, an S-band filter and a transmission line were selected. The intention was to fabricate these structures using the proposed and conventional methods.

Henceforth, the circuit structures fabricated using the proposed process chain will be referred as 'printed-plated structures' (i.e. printed-plated filter and printed-plated transmission line), and those fabricated using the conventional method will be referred as 'reference structures' (i.e. reference filter and reference transmission line).

The reasons behind the selection of S-band filter and transmission line for validation of the proposed concept are as follows:

- The performance of inkjet printed radio frequency identification devices (RFIDs) that operate in the megahertz $(\mathrm{MHz})$ range was already characterised under contemporary research initiatives [2,3]. Hence, it was decided to investigate a different frequency range and consequently a different application.

- The gigahertz $(\mathrm{GHz})$ range of frequencies is widely used in applications such as telecom, wireless IT, radar systems, radio astronomy etc. The partners in this research project, Thales Nederland B.V. and ASTRON, also focus extensively on $\mathrm{GHz}$ frequency applications. The fabrication of circuit structures for $\mathrm{GHz}$ applications is more challenging than for lower frequencies. This is due to the fact that the higher the frequency, the greater the role played by dimensional integrity of the structures in determining the RF performance. If it could be shown that the proposed process chain is suitable for the $\mathrm{GHz}$ frequency range, then it can be directly concluded that it is suitable for lower frequencies as well.

- The choice of a filter and a transmission line is logical, as it is relatively straightforward to compare the performances of the printed-plated and conventional structures. The key characteristics that can be compared are return loss $\left(S_{11}\right)$ and insertion loss $\left(S_{21}\right)$ and, in the case of the filter, the passband as well. 


\subsubsection{Approach}

An S-band filter and a $50 \mathrm{ohm}$ transmission line were designed. They were fabricated in the two different ways outlined in section 6.1.1. Subsequently, their performances were characterised by network analysis. It should be mentioned here that the filter design was not optimised, as this study was carried out primarily as a feasibility study, to find out the applicability of the proposed process chain in the fabrication of high-frequency RF circuit structures. It was not intended to fabricate a filter with an exact, pre-defined response.

The reproducibility of inkjet printed-electroless plated RF circuit structures was also evaluated by fabricating multiple specimens using the proposed fabrication methodology and comparing their performances.

In this chapter, the term 'printed structures' is used to denote inkjet printed silver seed layers; the term 'printed-plated structures', to denote inkjet printed and subsequently electroless plated layers; finally, the term 'specimen' is used to denote inkjet printedelectroless plated layers in combination with the substrate.

\subsection{Filter design}

A stripline S-band filter was designed using the software Advanced Design System (ADS, from Agilent Technologies). The functional elements of the stripline filter were realised by sections of high-impedance/low-impedance lines; a high-impedance line (narrow line) substituting an inductive element and a low-impedance line (broad line) substituting a capacitive element. This type of filter that is designed using only the sections of high- and low-impedance transmission lines is known as distributed element filter. The alternating low- and high-impedance design is referred to as stepped impedance design. A detailed discussion on distributed element filters can be found in Microwave Engineering by Pozar [4]. The designed transmission line had an impedance of $50 \mathrm{ohm}$, the standard value used in RF technology. 
Chapter 6

The design of the filter as well as the transmission line, along with the dimensions (rounded off to 2 significant digits), is depicted in figure 6.3(A). The S-band filter is at the top and the $50 \mathrm{ohm}$ reference transmission line is at the bottom. The transmission line was included in the design with the aim of locating the problem source, should the filter not yield the desired results; i.e. to identify whether the eventual discrepancy in RF performance was due to dimensional deviations of the printed-plated filter or the printing-plating process itself. Figure 6.3(B) shows the cross-section of the test design. The dielectric constant of the substrate material is 3.55 and its dielectric loss tangent is 0.0027 . The thickness of the circuit structures in this design is $17.5 \mu \mathrm{m}$. The filter and transmission lines are separated from the copper ground planes, which are at the top as well as the bottom of the cross-section, by a dielectric thickness of $1 \mathrm{~mm}$. Hence, the cross-section of the design shows 4 dielectric substrate layers, each $0.5 \mathrm{~mm}$ thick.

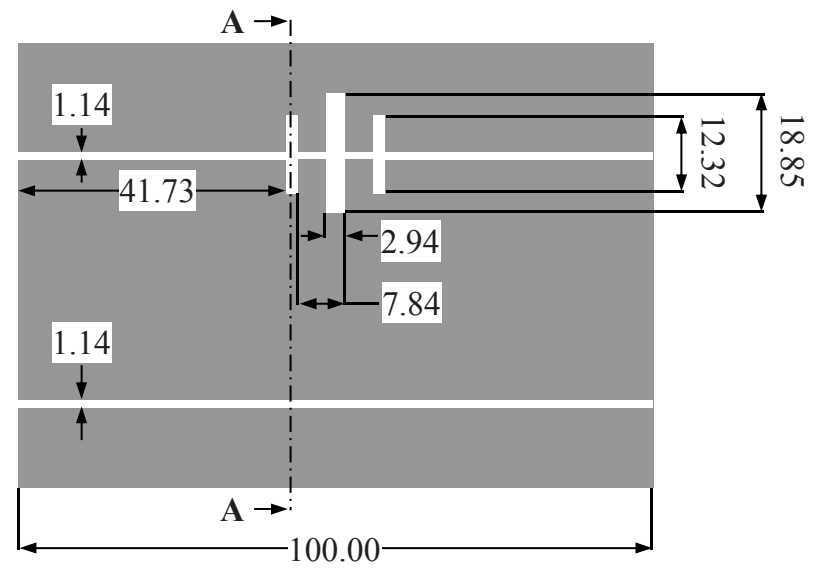

SECTION A-A

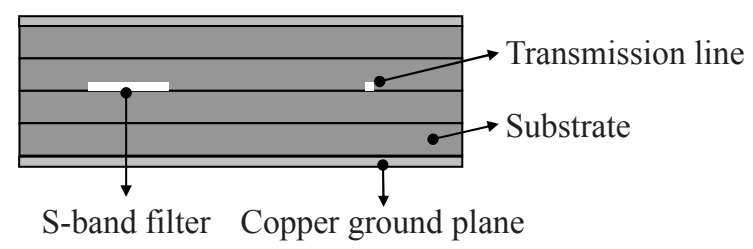

Figure 6.3:(A) S-band filter and $50 \mathrm{ohm}$ reference transmission line. The S-band filter is symmetric about horizontal and vertical axes passing through its geometric centre (all dimensions in $\mathrm{mm}$ ); (B) Schematic representation of the cross-section of the design. 


\subsection{Experiments}

\subsubsection{Materials and methods}

The materials and equipments used for this study are listed in the table below:

Table 6.1: Details of the raw materials and equipments relevant to this chapter.

\begin{tabular}{|c|c|c|}
\hline $\begin{array}{l}\text { Raw } \\
\text { material/equipment/process }\end{array}$ & Type/Name & Supplier \\
\hline Substrate & RO4003 & Rogers Corporation, USA \\
\hline Plasma etching setup & TePla 3067-E & $\begin{array}{l}\text { Technics Plasma GmbH, } \\
\text { Germany }\end{array}$ \\
\hline & 1.Harima silver nanopaste & 1. Harima Chemicals Inc., Japan \\
\hline Inkjet ink & $\begin{array}{l}\text { 2. Organic silver complex } \\
\text { compound (TEC-IJ-040) }\end{array}$ & $\begin{array}{l}\text { 2. InkTec Company Limited, } \\
\text { Korea }\end{array}$ \\
\hline Nozzle & $\begin{array}{l}\text { Piezo-actuated, } 60 \mu \mathrm{m} \\
\text { inner diameter }\end{array}$ & $\begin{array}{l}\text { MicroFab Technologies Inc., } \\
\text { USA }\end{array}$ \\
\hline Electroless copper plating system & Envision-2130 & Enthone Inc., USA \\
\hline Cleaning prior to plating & Neutraclean & Shipley Company, USA \\
\hline Network analysis & HP 8510 Network Analyser & Agilent Technologies, USA \\
\hline Surface roughness measurement & DEKTAK surface profiler & Veeco Instruments Inc., USA \\
\hline Electron microscope & JSM-6400 & JEOL Limited, Japan \\
\hline
\end{tabular}

\subsubsection{Initial experimental trials}

Prior to printing of the designed RF circuit structures, inkjet printing and electroless plating trials were carried out with the objective of identifying the optimal inkjet printing parameters and the plating duration, respectively. These trials were done on substrates subjected to plasma etching, for reasons outlined in chapter 4 . 
Chapter 6

\subsubsection{Inkjet printing}

The parameters of the inkjet printing process were optimised to ensure the continuity of the printed structures, without inducing pronounced cross-sectional variations due to droplet overlapping. This step was necessary as the droplet spreading depends on the surface morphology of the substrate, and is hence case-specific. The droplet spacing (pitch) in $\mathrm{X}$ and $\mathrm{Y}$ directions was the outcome of this optimisation process. The bipolar waveform described in chapter 3 was chosen to actuate the nozzle. The distance between the nozzle tip and the substrate was maintained at $1.5 \mathrm{~mm}$. The droplet size after spreading on the substrate was approximately $175 \mu \mathrm{m}$. The droplet pitch values thus determined were used for all the subsequent printing trials.

\subsubsection{Electroless plating}

Initial electroless plating trials were carried out on printed tracks in order to determine the plating rate. The plating rate was mentioned as 2 to $2.5 \mu \mathrm{m} / 30$ minutes in the datasheet provided by the supplier [5] of the plating solution. Nevertheless, it was decided to ascertain the same independently, as the rate can vary depending on the seed layer, which acts as the catalyst for plating reactions. In general, the time required to obtain a continuous copper layer is highly influenced by the local surface density of the silver particles and the composition of the plating bath. The plating bath composition as well as the pre- and post-plating cleaning steps was the same as discussed in chapter 4 . The evolution of the microstructure as a result of the plating process was studied using SEM. The thickness of the printed-plated structures was measured by surface profiling.

\subsubsection{Specimen preparation}

The first step in specimen preparation was to program the coordinates of the S-band filter and the transmission line following a printer-specific syntax. Apart from the coordinates, other parameters such as the droplet spacing (pitch), which was determined earlier, the direction of printing and the velocity of substrate holder movement were also specified in the program. 
Substrates measuring $100 \mathrm{~mm} \times 100 \mathrm{~mm}$ (length $\times$ width), were cut. They were then cleaned with ethanol and dried in a convection oven. Inkjet printing of the seed layer was the next step. All the printed structures were only one layer thick, as their primary function was to act as seed layers and not as functional RF circuit structures by themselves. The printed structures were then sintered in a convection oven for 60 minutes at $210{ }^{\circ} \mathrm{C}$ as specified by the ink supplier. This temperature is sufficient to enable the onset and development of nanoparticle growth and grain boundary migration, leading to continuity in the printed structures. However, it should be noted that the sintered tracks were not $100 \%$ dense - pores were observed, resulting from the shrinkage of the printed material during the sintering process. Similar observations have been reported in [6-8].

The next step in the specimen preparation was electroless plating. Even though the design thickness of the RF circuit structures was $17.5 \mu \mathrm{m}$, it was decided to aim for a plating thickness of just 2.5 to $3 \mu \mathrm{m}$, as the 'skin effect' is quite pronounced in the frequency range being dealt with, thereby rendering thicker conductive structures superfluous. In brief, the skin effect can be explained as the tendency of the electromagnetic waves to travel along the 'skin' or the surface of a conductor, at high frequencies. As the frequency increases, the penetration depth of the electromagnetic field into the conductor decreases. Calculations were done to ascertain the skin depth, using equation (6.1) [9], which is widely used in RF theory and technology:

$\delta=\frac{1}{\sqrt{\pi f \mu \sigma}}$, Eqn. (6.1)

where $\delta$ is the skin depth (m), $f$, the frequency of the electromagnetic wave (Hz), $\mu$, the magnetic permeability of the conductor $\left(\mathrm{H}_{\mathrm{m}} \mathrm{m}^{-1}\right.$ or $\left.\Omega . \mathrm{s}^{\mathrm{m}} \mathrm{m}^{-1}\right)$ and $\sigma$, the conductivity of the conductor $\left(\Omega^{-1} \cdot \mathrm{m}^{-1}\right)$. From these calculations, the skin depths for copper in the S-band range were found to be $1.46 \mu \mathrm{m}$ at $2 \mathrm{GHz}$ and $1 \mu \mathrm{m}$ at $4 \mathrm{GHz}$. The combined thickness of the final bi-material printed-plated circuit structures was about $4 \mu \mathrm{m}$.

The higher-than-required thickness of the circuit structures was intended to compensate the higher resistivity of the printed-plated structures $(3 \mu \Omega . \mathrm{cm})$ when compared to bulk 
Chapter 6

copper $(1.72 \mu \Omega . \mathrm{cm})$. The higher thickness of the printed-plated structures provides greater skin thickness for the RF wave. The resistivity was calculated from resistance measurements, assuming that the cross-section of the printed-plated structure was uniform. However, the actual cross-section dimensions were not uniform owing to the nature of the fabrication process combination used. Figure 6.4 shows a SEM micrograph of the cross-section of a printed-plated structure. The thicknesses of circuit structures after inkjet printing as well as after printing and plating were obtained from their crosssection images.

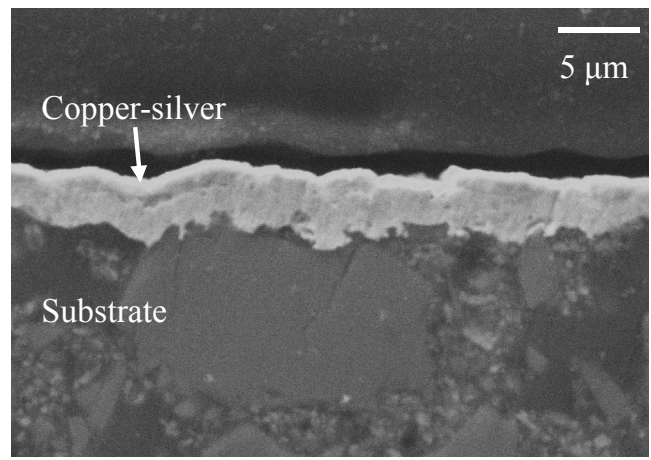

Figure 6.4: SEM micrograph of a printed-plated structure.

To compare the performance of the printed-plated structures with that of the structures fabricated using conventional lithography-based process, the same RF test structures were fabricated on a copper clad RO4003 substrate. For this purpose, the process chain depicted in figure 6.2 was used.

\subsubsection{RF performance characterisation}

The substrates containing circuit structures fabricated using 2 different methods were separately sandwiched between 2 substrate layers on one side and 1 substrate layer on the other (as shown in figure 6.3(B)). Then, Sub-Miniature version A (SMA) connectors, which are coaxial RF connectors, were mounted on each specimen. The specimen and connectors are shown in figure 6.5. In this figure, there are three pairs of SMA connectors mounted on the setup. The third pair of connectors was used with the intention of characterising a third RF test structure (apart from the S-band filter and the 
$50 \mathrm{ohm}$ line), which is not discussed in this thesis. To eliminate any air gap between the dielectric substrates or between the RF circuit structures and the adjacent substrate, this setup was clamped tightly between two aluminium plates. Finally, the two specimens were subjected to network analysis to evaluate their performance characteristics.

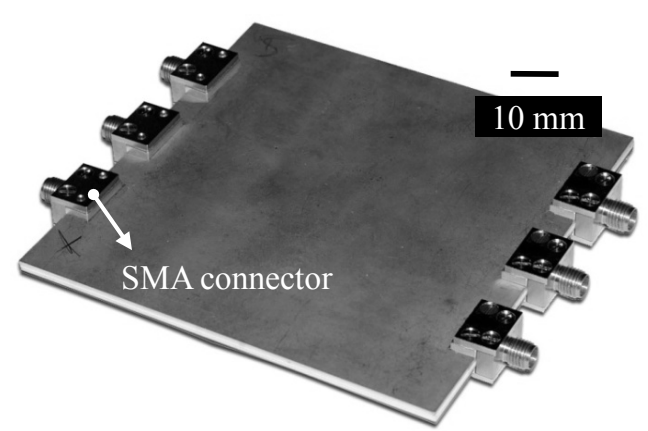

Figure 6.5: Specimen for network analysis.

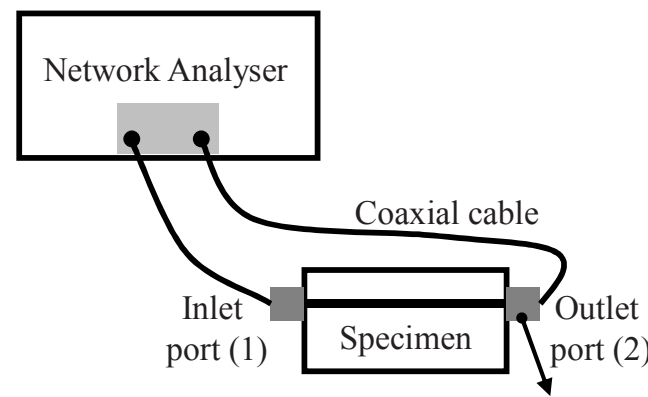

SMA connector

Figure 6.6: Schematic of the network analysis setup.

Figure 6.6 shows the measurement schematic. The incident RF wave from the network analyser enters the test specimen (i.e. S-band filter or transmission line) via port 1. Let $a_{1}$ be the voltage of the incident wave, $b_{2}$, the voltage available at the outlet port 2 , and $b_{1}$, the voltage reflected back and hence exiting from port 1 . From these 3 variables, the scattering parameters or S-parameters, which are commonly used to characterise RF performance, can be determined. The S-parameters of interest to this study are insertion loss $\left(S_{21}\right)$ and return loss $\left(S_{11}\right)$. They can be defined as, respectively [10,11]:

$S_{21}=b_{2} / a_{1}$

Eqn. (6.2) 
Chapter 6

$S_{11}=b_{1} / a_{1}$

Eqn. (6.3)

Usually, the S-parameters are represented in decibels $(\mathrm{dB})$. To convert the S-values from linear magnitude to logarithmic values, the following equations are used:

$S_{11}(d B)=20 \log \left[S_{11}\right.$ (magnitude) $]$

Eqn. (6.4)

$S_{21}(d B)=20 \log \left[S_{21}\right.$ (magnitude)]

Eqn. (6.5)

Insertion loss $\left(S_{21}\right)$ is a measure of energy lost during the transmission of a signal through the specimen under analysis, and return loss $\left(S_{11}\right)$ accounts for the signal reflection due to e.g. impedance mismatch.

\subsubsection{Reproducibility of the proposed fabrication method}

Once it became clear that the performance of the printed-plated structures is comparable to the conventionally fabricated ones, the question that arose immediately was whether or not the proposed fabrication method is reproducible. To assess this, 4 more S-band filter specimens were inkjet printed and electroless plated. While Harima silver nanopaste was used in the previous case, the organic silver complex compound was used in this case to print the filters. This was done to check if a change in seed layer has any effect on RF performance. No modification was made to the plating process, including the duration. The consistency of the passbands of these filters was studied.

\subsection{Results and discussion}

\subsubsection{Inkjet printing}

Figure 6.7 shows the substrate on which droplets with different $\mathrm{X}$ and $\mathrm{Y}$ pitch values were deposited. Microscopic studies indicated that the optimal pitch values for both $\mathrm{X}$ and $\mathrm{Y}$ directions were $0.15 \mathrm{~mm}$. There was indeed droplet overlapping in both the directions; it was kept to a minimum though, in order to keep cross-sectional variations limited to a smaller area. Highly uneven, undulating cross-sections lead to a longer path the RF wave has to travel, resulting in deviations from the desired RF performance. Increasing the pitch to eliminate overlapping led to scalloped edges, which is also not 
favourable to good RF behaviour. Hence, a compromise was found by choosing the abovementioned pitch.

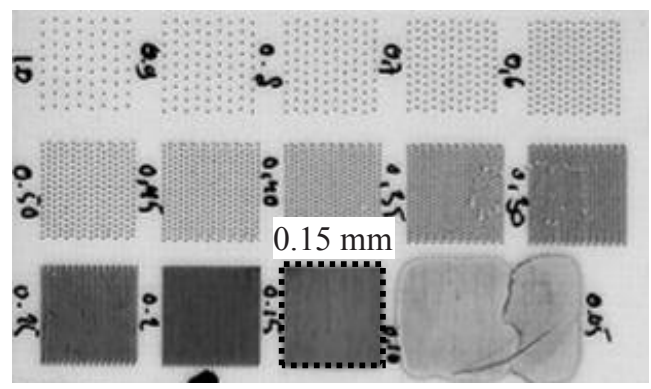

Figure 6.7: RO4003 substrate on which droplet optimisation was carried out. The chosen (optimal) pitch value and the corresponding printed structure are indicated. The Harima silver nanopaste was used for this optimisation process.

\subsubsection{Electroless plating}

The electroless copper plating rate was found, based on surface profiling, to be about $1.5 \mu \mathrm{m} / \mathrm{hr}$. This value is much lower than that specified in the datasheet of the supplier. As mentioned earlier, the nature and composition of the seed layer plays an important role in determining the rate of plating.

\subsubsection{Fabrication of RF circuit structures}

The S-band filter and transmission line were inkjet printed using the optimised droplet spacing, and were subsequently electroless plated. Since the plating rate was found to be $1.5 \mu \mathrm{m} / \mathrm{hr}$, the printed structures were immersed in the electroless plating bath for 120 minutes, in order to obtain a copper layer thickness of about $3 \mu \mathrm{m}$. Figure 6.8 shows the printed-plated RF structures on a RO4003 substrate. The same structures were also fabricated by the conventional method, the specifics of which are not discussed here. 
Chapter 6

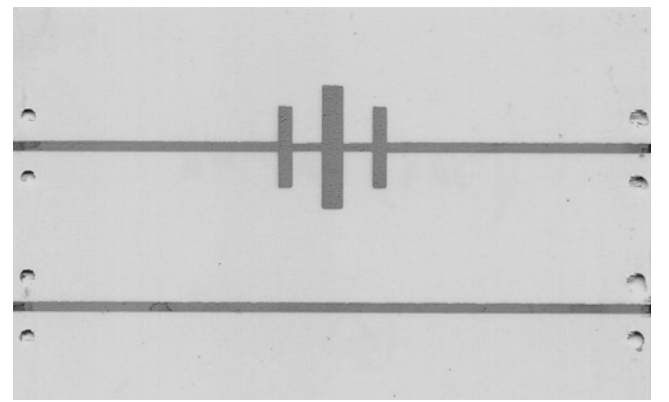

Figure 6.8: Inkjet printed-electroless plated RF structures on a RO4003 substrate.

\subsubsection{RF measurements}

6.4.4.1. Comparison of printed-plated and reference circuit structures

During the circuit analysis, the frequency was increased from $1 \mathrm{GHz}$ to $10 \mathrm{GHz}$ in steps of $45 \mathrm{MHz}$. It became clear from the measurements that the centre frequency of the reference (conventional) filter was $2.305 \mathrm{GHz}$. Figure 6.9 compares the return losses of the reference and printed-plated filters. The larger the $S_{11}$ is in negative $\mathrm{dB}$, the smaller is the reflection, and hence the lower the return loss.

It can be seen from figure 6.9 that the performance of the printed-plated filter is comparable to that of the reference filter. It can also be seen from this figure that the printed-plated filter is resonant at two different frequencies i.e. there are 2 nulls, and the reflection increases slightly between these frequencies. This has to do with the design of the filter, shown in figure 6.3. Preliminary simulations done during the filter design also exhibited 2 nulls around the centre frequency. However, the reference filter with the same design exhibits just one null. The exact reason(s) for this mismatch is unclear. 


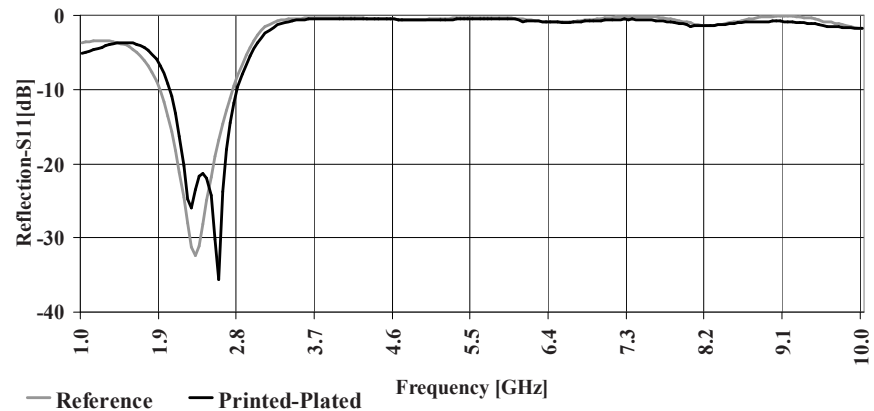

Figure 6.9: Comparison of return losses of the two S-band filters.
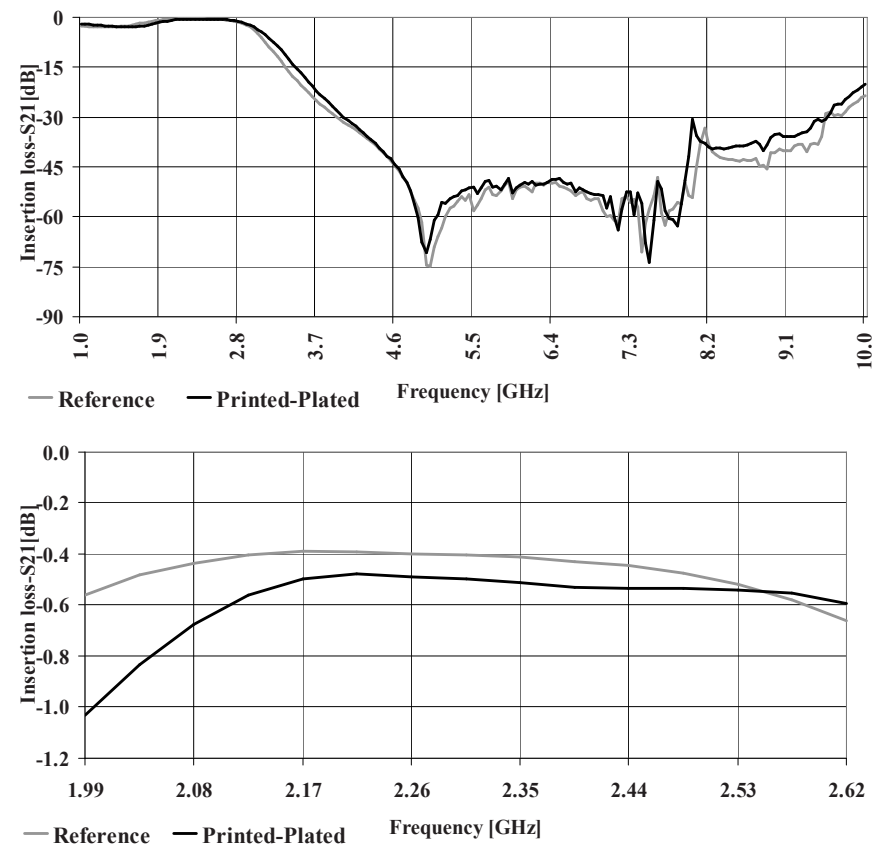

Figure 6.10: (A) Comparison of insertion losses of the two S-band filters; (B) Detailed view of insertion losses of the two S-band filters around the centre frequency.

Figure $6.10(\mathrm{~A})$ compares the insertion losses of the two filters. A value of $0 \mathrm{~dB}$ indicates full transmission of the RF wave. As the insertion drops, a smaller proportion of the signal is transmitted, and the value in terms of negative $\mathrm{dB}$ becomes larger. In this case also, the performance of the printed-plated filter is almost identical to the reference filter. Figure 6.10(B) shows the insertion loss of the filter in the region of interest i.e. around the centre frequency. It can be seen in this figure that the insertion loss for the 
Chapter 6

printed-plated filter is close to $0 \mathrm{~dB}(-0.496 \mathrm{~dB}$, to be exact) for the centre frequency. The corresponding value for the reference filter is, as shown in the figure, $-0.402 \mathrm{~dB}$. The average insertion loss over the passband for the printed-plated filter is $-0.534 \mathrm{~dB}$, as against $-0.440 \mathrm{~dB}$ for the reference filter.

Apart from the S-band filters, the $50 \mathrm{ohm}$ transmission lines were also tested. The performance of the printed-plated transmission line and the reference transmission line are shown in figures 6.11 and 6.12. From figure 6.12, it is clear that the insertion losses for these two types of transmission lines are more or less in agreement with each other, while figure 6.11 shows some difference in their responses with respect to the return loss. In both cases, the transmission line fabricated using the conventional method showed a slightly superior performance when compared to printed-plated line. The insertion loss comparison in this case is very similar to that of the results presented in [12], in which the response curve of a printed transmission line was just under that of a conventionally fabricated transmission line, indicating a slightly inferior performance of the former. However, the difference is marginal and can be neglected.

Interestingly, figures 6.11 and 6.12 depict wave-like responses of the transmission lines. This is due to the fact that the network analyser was connected using coaxial cables to the coaxial SMA connectors, which were, in turn, mounted on the striplines. Calibration of the network analyser was done up to, but not including, the SMA connectors. This transition from coaxial to stripline at the input, and stripline to coaxial at the output, is considered the reason for the periodic distortion in the responses of the transmission lines. Also, a shift between the peaks of the two responses can be seen in figures 6.11 and 6.12, which can be attributed to the minor differences in the transmission lines' dimensions. This shift can be seen in the responses of the filters as well, shown in figures 6.9 and 6.10 , due to the same reason.

The dimensions of the printed-plated filter as well as the printed-plated transmission line deviated from the design values. In the case of the filter, the deviation varied from $0.3 \%$ to $8 \%$, depending on the dimensions of individual lines that make up the filter. In the case of the transmission line, the deviation was about $6 \%$. The cause for these deviations is that with fixed, optimised droplet spacing, it was not possible to realise the 
exact design dimensions i.e. the design dimensions were not exact multiples of a single track width. As explained before, marked overlapping of adjacent tracks was not desired; hence, deviations from the design values were deemed acceptable. It can also be seen from figures 6.11 and 6.12 that the wave-like patterns exhibited by the reference transmission line are fairly regular and well-defined, whereas the response of the printed-plated transmission line is irregular. This can be attributed to the consistent cross-section of the reference transmission line. On the other hand, the cross-section of the printed-plated line in terms of thickness and width varied along its length. In spite of optimisation of the printing parameters, this is unavoidable, as the inkjet printed seed layer is bound to have such undulations resulting from overlapping of droplets.

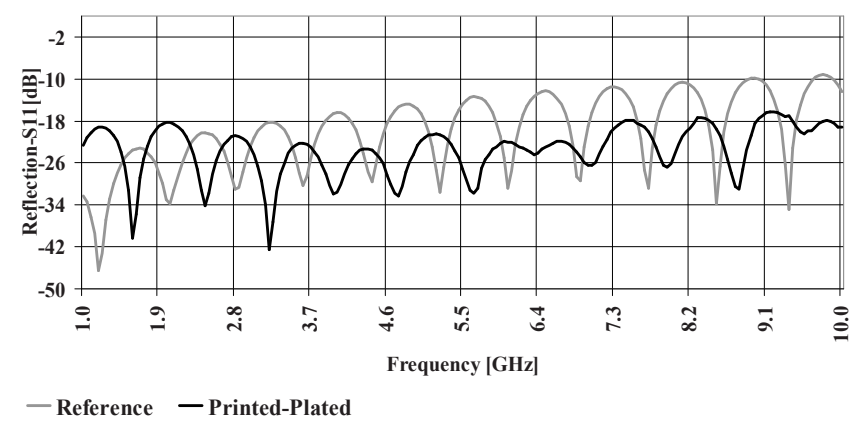

Figure 6.11: Return losses of the $50 \mathrm{ohm}$ transmission lines.

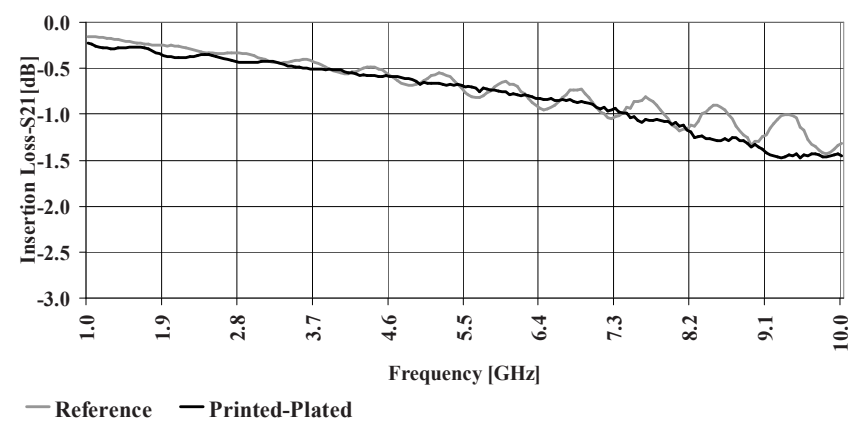

Figure 6.12: Insertion losses of the $50 \mathrm{ohm}$ transmission lines.

\subsubsection{Reproducibility of the proposed fabrication method}

Figure 6.13 depicts the passband performance of the 4 filter specimens (numbers 1 to 4 ) printed using the organic silver complex compound. This figure also shows the 
performance of the specimen printed using Harima silver nanopaste (number 0), which was already discussed. It is clear from this figure that the average insertion losses of all the filters in the passband is well below $1 \mathrm{~dB}$, and are in agreement with each other. There are of course variations, but they are negligible. It can also be seen that the performances of filters 1 to 4 are marginally better than that of filter 0 . This is due to minor differences in their dimensions. These 2 different ink types exhibit different spreading behaviour on RO4003 substrate. Even though the droplet spacing was individually optimised before printing filters using these 2 inks, dimensional variations did result.

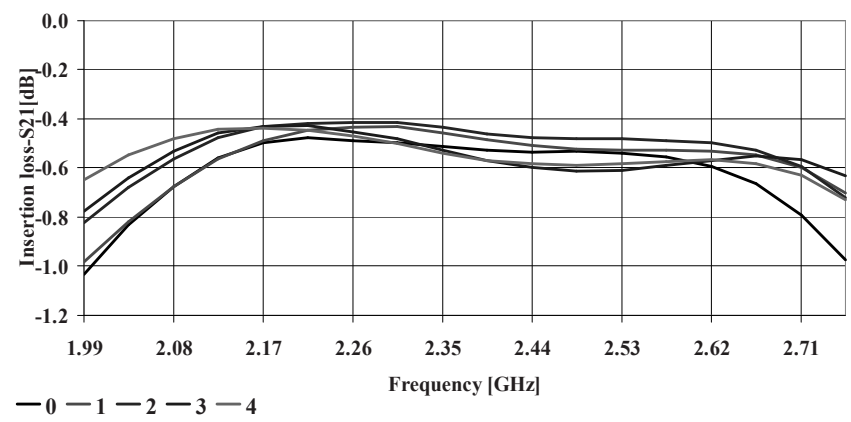

Figure 6.13: Detailed view of insertion losses of S-band filters around the centre frequency; 0 represents the previously characterised filter and 1 to 4 represent the subsequently fabricated filters.

\subsection{Conclusions}

The most important outcome of this study is the successful fabrication of an S-band filter, using a combination of inkjet printing and electroless plating, with a response comparable to that of a conventional filter. Moreover, the inkjet printing-electroless plating-based process chain that is proposed in this thesis has been validated. As already discussed in chapter 4, Scotch tape tests indicated neither cohesive nor adhesive failure of inkjet printed-electroless plated circuit structures. Based on all these outcomes, it can be concluded that this process chain exhibits great potential for commercial fabrication of (RF-) electronic circuits. 


\subsubsection{Reduction of manufacturing time and process scalability}

As shown in figure 6.1, there are 4 main steps in the proposed fabrication method, namely, plasma treatment, inkjet printing, electroless plating and functional characterisation. The last step is a general process step, which is integral to the conventional fabrication method as well. Usually, in an industrial environment, such a process step is applied only to a few specimens from a batch for quality control. Hence, there are 3 main steps that should be repeated for each and every product produced using the proposed process chain. The total process time is relatively high in this case, especially due to the long plating time, and has to be brought down for successful commercial implementation. It is possible to reduce the process time, due to the following reasons:

- To print the S-band filter as well as the transmission line, the printer used during this study took about 12 minutes. If industrial inkjet printers are used, the printing time will be much lower, due to faster drives and simultaneous printing by multiple nozzles.

- The number of substrates that can be simultaneously electroless plated is limited only by the size of the plating bath and the minimum distance that should be maintained between two adjacent substrates, which is generally in the order of $10 \mathrm{~mm}$. This means, with a suitable plating bath, tens or even hundreds of substrates could be simultaneously plated, reducing the process time per substrate drastically. Moreover, higher plating rates of up to $7.2 \mu \mathrm{m}$ per hour have been reported [13]. So, the usage of plating baths yielding such high plating rates will quicken the process even further.

The inkjet printing-electroless plating based fabrication method is readily scalable upward, as both inkjet printing and electroless plating are widely used in the industry for small, medium as well as large scale fabrication. Moreover, this process combination can be used to fabricate circuit structures on rigid as well as flexible substrates. 
Chapter 6

\subsection{References}

[1] "Inkjet PCB production project”, New Energy and Industrial Technology

Development Organization (NEDO), Japan:

http://www.nedo.go.jp/english/activities/1_sangyo/2/p03026e.html (accessed: February 2010).

[2] R. Vyas, A. Rida, L. Yang and M.M. Tentzeris: "Design and development of a novel paper-based inkjet-printed RFID-enabled UHF (433.9 MHz) sensor node", Asia-Pacific Microwave Conference Proceedings, APMC, Art. No. 4554641, 2007.

[3] L. Zheng, S. Rodriguez, L. Zhang, B. Shao and L.-R. Zheng: "Design and implementation of a fully reconfigurable chipless RFID tag using inkjet printing technology", Proceedings of the IEEE International Symposium on Circuits and Systems, Art. No. 4541720, p. 1524, 2008.

[4] D.M. Pozar: "Microwave engineering", $2^{\text {nd }}$ edition, John Wiley \& Sons, Inc., 1998.

[5] Enthone Envision EC2130 Datasheet, Enthone Inc., USA.

[6] S.P. Wu, K.C. Yung, L.H. Xu and X.H. Ding: "Fabrication of polymer silver conductor using inkjet printing and low temperature sintering process", IEEE Transactions on Electronics Packaging Manufacturing, Vol. 31, No. 4, p. 291, 2008.

[7] N.A. Luechinger, E.K. Athanassiou and W.J. Stark: “Graphene-stabilized copper nanoparticles as an air-stable substitute for silver and gold in low-cost ink-jet printable electronics”, Nanotechnology, Vol. 19, No. 44, Art. No. 445201, 2008.

[8] B.-K. Park, D. Kim, S. Jeong, J. Moon and J.-S. Kim: "Direct writing of copper conductive patterns by ink-jet printing", Thin Solid Films, Vol. 515, No. 19, p. 7706, 2007.

[9] B.K. Sen and R.L. Wheeler: "Skin effects models for transmission line structures using generic SPICE circuit simulators":

http://www.wheeler.com/technology/technicalpaper2/technicalpaper2.pdf (accessed: February 2010).

[10] “Agilent AN 154 S-parameter design (application note)", Agilent Technologies, Inc., USA, 2006.

[11] http://www.microwaves101.com/encyclopedia/sparameters.cfm (accessed:

February 2010). 
[12] M. Mantysalo and P. Mansikkamaki: “An inkjet-deposited antenna for $2.4 \mathrm{GHz}$ applications", International Journal of Electronics and Communications, Vol. 63, No. 1, p. 31, 2009.

[13] C.-Y. Kao and K.-S. Chou: "Electroless copper plating onto printed lines of nanosized silver seeds", Electrochemical and Solid-State Letters, Vol. 10, No. 3, p. D32, 2007. 
7. Conclusions and Recommendations

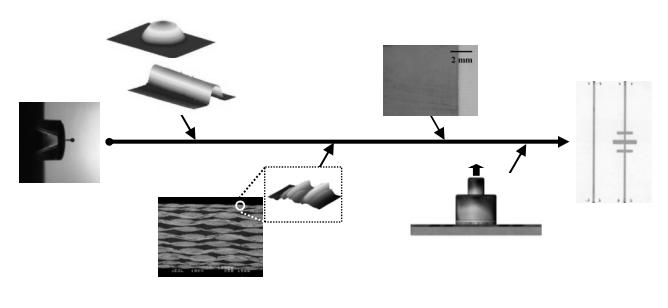


Chapter 7

\subsection{Conclusions}

The main conclusions of this thesis are summarised thus:

1. For inkjet printing to be used to print structures on rigid, reinforced PCB substrate materials, process-specific surface morphology of the substrates is vital. Printing with accuracy on such substrates is possible if they are pre-conditioned accordingly. The surface morphology of conventional PCBs, from which the inherent copper cladding was etched away, is generally unfavourable to the printing accuracy. The same conclusion can be drawn about the surface morphology of substrates on which crimping of fibre reinforcements occurred during their production.

2. An inkjet printing-based process chain was proposed for the fabrication of functional conductive structures on printed circuit board materials. This process chain includes techniques to pre-condition the substrate for improved adhesion (without affecting the printability), fabricating seed structures, developing the seed structures into functional circuit structures, and finally, characterising the same. The individual elements i.e. techniques of the proposed process chain fulfil the following functionalities:

- Plasma treatment: It is an effective way to create functional groups on the surface of a polymeric PCB substrate. These functional groups aid chemical bond formation with the (inkjet-) deposited metal. Additionally, plasma treatment also enhances surface roughness, which improves mechanical interlocking between the deposited metal and the substrate.

- Inkjet printing: The central component of the proposed process chain, inkjet printing is the technique chosen to fabricate the seed structures on a PCB substrate. Even though it is possible to inkjet print multiple layers one on top of the other (i.e. stacking), it was demonstrated that this approach has some inherent pitfalls. Lower conductivity values, problems in soldering, and formation of pores and cracks due to sintering are some of the pitfalls. Hence, the approach followed in this thesis involves inkjet printing of only one (seed) 
layer. The inkjet printing process is already applied on an industrial scale, with the availability of high-speed, multi-nozzle printing systems.

- Electroless plating: This technique was drafted into the process chain as a suitable method to impart the desired thickness to the inkjet printed seed structures. This technique, like inkjet printing, is readily scalable upwards. The number of substrates containing inkjet printed seed layers that can be electroless plated simultaneously is dependent only on the size of the plating bath, and the minimum distance that should be maintained between two adjacent substrates. Both these factors can be addressed readily in an industrial environment.

- Characterisation: RF and mechanical characterisation are the two components that make up this process step. Even in a conventional PCB fabrication setup, mechanical and RF characterisation is usually done to ascertain the quality of the end product.

3. The proposed process chain was validated with a practical application in the form of RF circuit structures. An important offshoot of this validation is the demonstration of the applicability of inkjet printing for high-radio-frequency applications, beyond that of the oft-discussed RFID tags. An S-band filter fabricated within the framework of this research, exhibiting a comparable performance to that of a conventionally fabricated counterpart, serves as a proof-of-concept.

4. It is readily possible to apply the proposed process chain to lower i.e. $\mathrm{MHz}$ and $\mathrm{kHz}$ range frequencies as well. The main difference between the demonstrated S-band application and lower frequency applications such as RFID tags is in the dimensions of the printed structures. Typically, lower frequencies demand broader and thicker lines. Since this process chain requires inkjet printing of just a single seed layer, printing wider structures should not pose any problems. The case for this process chain is emphasized by the less stringent edge and cross-sectional accuracies of the conductive circuit structures needed for lower frequency applications. 
Chapter 7

5. A typical electronic circuit includes active as well as passive components such as diodes and resistors. Inkjet printing is seen as a technique that can be used to fabricate these components as well. However, electroless plating has no role to play in the fabrication of these components. Hence, it is necessary to inkjet print multiple layers one on top of the other i.e. stacking, to attain the desired thickness of these 3D structures. In this thesis, experiments were done to find out the best approach to inkjet print multiple layers. It was concluded that the wet-on-partial-dry approach, which involves heating of the substrate while printing, is more suitable than the wet-on-wet approach.

6. Finally, with the aid of the pull-off test method, it was shown that surface roughness plays a major role in determining the interfacial strength in an inkjet printed metalpolymeric substrate system. At the same time, pronounced surface roughness will affect the dielectric properties of the substrate, besides negatively influencing the printing accuracy. Moreover, a rougher substrate leads to a longer path that the electrons need to travel to transmit energy. As a rule-of-thumb, for (RF-) electronics applications, the higher the frequency, the greater is the influence of substrate surface roughness on the performance. Therefore, a case- and application-specific trade-off between substrate surface roughness and the desired performance is necessary for optimum results. 


\subsection{Recommendations}

Based on the conclusions drawn from this thesis, some recommendations are presented here for follow-up research:

1. A functional electronic circuit (demonstrator) such as an RLC (resistance-inductancecapacitance) circuit can be fabricated using the proposed process chain. Its performance should be compared with that of a conventionally fabricated circuit or simulation results. This would demonstrate whether or not the proposed process chain can be integrated into the larger domain of electronics fabrication.

2. The proposed process chain can be modified to include laser ablation in the place of plasma treatment. Laser ablation is a selective surface patterning process that can be used to create 'anchoring locations' on the substrate. These anchoring locations, typically holes, can be in the order of a few $\mu \mathrm{m}$ in diameter and 1 or $2 \mu \mathrm{m}$ in depth. By ablating an array of such holes on the substrate prior to printing, mechanical interlocking of the printed structure to the substrate is promoted. This has a positive effect on interfacial adhesion.

During the course of this research, laser ablation of polymeric substrate materials was also performed to impart roughness patterns on them. A detailed discussion about these experiments is not provided in this thesis, as the complete set of results is not available due to time constraints. Nevertheless, selected results from the experiments done during this research are provided in Appendix-B, to serve as a guideline for future research direction.

An advantage of the laser ablation method over plasma treatment is that it is highly selective, and the equipment used for laser ablation can be programmed in such a way that the anchoring patterns can be varied locally on the substrate. This can be done to compensate for the unique spreading behaviour of each ink type on a given substrate material. Moreover, laser ablation does not modify the chemical structure of the substrate surface significantly, which is not the case with plasma treatment. This is beneficial, since the ink-substrate interaction for a given substrate is ink-specific; a 
Chapter 7

plasma-modified chemical structure might be favourable for one type of ink, while at the same time proving to be undesirable in the case of the other.

This modified process chain can be applied to fabricate the abovementioned RLC circuit. The conductive circuit structures, capacitors and inductors can be fabricated by the inkjet printing-electroless plating combination. Subsequently, the same process chain, but without the electroless plating step, can be applied to fabricate resistors. In this case, inkjet printing of multiple layers is necessary to attain the desired thickness of the resistors.

3. The quantification of interfacial adhesion done in this thesis can be taken a step further to include the influence of thermal, mechanical and environmental stress on interfacial strength of an inkjet printed structure-polymeric substrate system. This will provide an insight into the reliability and robustness of inkjet printed structures in conditions in which they will be applied. 


\section{Appendix-A}

\section{Coefficient of determination $\left(\mathbf{R}^{2}\right)$}

In the case of simple regression analysis, the coefficient of determination measures the proportion of the variance in the dependent variable explained by the independent variable [1]. It indicates how well the model fits the actual data [2]. It is defined as the ratio of two sums of squares, and is calculated by:

$R^{2}=S S R / S S T$,

where $S S R$ is the sum of squares due to regression and SST is the total sum of squares. Sum of squares refers to the sum of squared deviations between the predicted values and the mean $(S S R)$, or the sum of squared deviations between the actual (observed) values and the mean $(S S T)$ [3]. Considering a dataset with actual values $y_{i}$ and model values $f_{i}$, SSR and SST can be calculated as:

$\operatorname{SSR}=\left(f_{i}-\bar{f}\right)^{2}$, and

$S S T=\left(y_{i}-\bar{y}\right)^{2}$

The coefficient of determination can take up values between 0 and $100 \%$. Values closer to $100 \%$ indicate a good fit of the predicted values. For example, an $R^{2}$ value of $90 \%$ indicates that $90 \%$ of the total variation in the outcome can be explained by the regression equation that relates the process variables (predictors) and the outcome.

\section{Predicted coefficient of determination $\left(R^{2}\right.$ (predicted))}

Predicted coefficient of determination is used in regression analysis to indicate how well the model predicts responses for new observations. It can prevent over-fitting the model and can be useful for comparing models. Over-fitting refers to models that appear to 
Appendix- $A$

explain the relationship between the predictor and response variables for the data set used for model calculation but fail to provide valid predictions for new observations [2].

Predicted $R^{2}$ is calculated by systematically removing each observation from the data set, estimating the regression equation, and determining how well the model predicts the removed observation. Predicted $R^{2}$ ranges between 0 and $100 \%$ and is calculated from the PRESS (Prediction Residual Error Sum of Squares) statistic. Larger values of predicted $R^{2}$ indicate models of good predictive ability [2].

$R^{2}($ predicted $)=[1-($ PRESS $/ S S T)] \times 100$,

where the PRESS statistic is used to summarise the fit of a particular model in a sample of observations that were not used to estimate the model parameters. It can be computed as the sums of squares of the prediction residuals for those observations [4].

\section{References}

[1] M.P. Allen: "Understanding regression analysis", Springer, USA, 1997.

[2] http://www.minitab.com/en-US/support/answers/answer.aspx?ID=983 (accessed:

February 2010).

[3] http://www.statistics.com/resources/glossary/c/coeffdeterm.php (accessed: February 2010).

[4] http://www.statsoft.com/textbook/statistics-glossary (accessed: February 2010). 


\section{Appendix-B}

\section{Laser ablation of FR4 substrates}

In chapter 5, a pressing-based method to roughen substrates prior to printing, without modifying them chemically, was presented. Another method to produce roughness patterns on the substrate prior to printing, without modifying it chemically was also experimented with. This method is the pulsed laser ablation method.

As already mentioned in chapter 1, laser ablation involves removal of material by a laser beam that transfers its energy to the target i.e. substrate. In the pulsed mode of operation, energy from the laser beam is transferred to the material in the form of pulses, thereby heating up the material locally in a very short time. This prevents the dispersion of heat into the bulk of the material. This method is very attractive as a candidate for substrate pretreatment, due to the following reasons:

- Possibility to pattern the substrate in a case-specific, ink-specific manner without chemically modifying the substrate.

- Contemporary research initiatives consider pulsed laser as a technique that can also be used to sinter metallic (nano-) particles deposited by inkjet printing [1,2]. This augurs well for the future, as the same laser equipment could potentially be used to pre-treat the substrate and, subsequent to inkjet printing, sinter the metallic ink as well.

- Laser is also widely used in the PCB industry for via-drilling. Pudas et al [3] discussed the PCB patterning as well as via drilling using a $355 \mathrm{~nm}$ wavelength pulsed laser setup. They also highlighted that a single laser setup can be used for a variety of operations within the PCB industry. Even though the laser type and wavelength used in the cited studies [1-3] are different, there is a possibility to find a common ground. Such an attempt might lead to further shortening of the complete PCB fabrication chain. 


\section{Appendix-B}

With these prospects as motivation, laser ablation experiments were carried out on FR4. The objective was to create roughness patterns in the form of ablated holes, with diameter less than $10 \mu \mathrm{m}$ and depth less than $3 \mu \mathrm{m}$. By ablating an array of such holes, anchoring locations can be created for the deposited metallic structure, which will promote interfacial adhesion. It was intended to vary three parameters governing the array viz. pitch $\left(\mathrm{p}_{\mathrm{x}}\right.$ and $\left.\mathrm{p}_{\mathrm{y}}\right)$, hole diameter (D) and hole depth (d), so as to identify their optimum values for good adhesion between the silver structure and FR4 substrate. These parameters are illustrated in figure 1 .
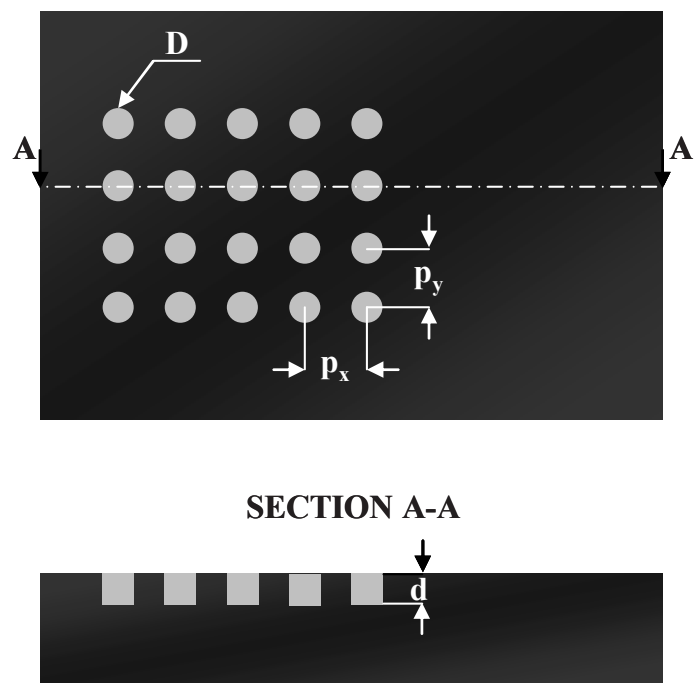

Figure 1: Schematic of the array of laser ablated holes and the associated parameters.

The details of the laser setup used for pulsed laser ablation experiments are given in table 1 . Thales Nederland B.V. is the owner of this setup. Table 2 lists the optimised ablation parameters. The optimisation was based on a statistical DoE, similar to the one used for plasma treatment, discussed in chapter 4. 
Table 1: Details of the laser ablation setup.

\begin{tabular}{|l|l|}
\hline Setup & Hitachi H-Mark-50L \\
Type of laser & $\mathrm{UV}$ \\
Wavelength & $355 \mathrm{~nm}$ \\
Medium & $\mathrm{YVO}_{4}$ \\
Beam diameter & $50 \mu \mathrm{m}$ \\
Maximum average power & $10 \mathrm{~W}$ \\
Maximum pulse duration & $30 \mathrm{~ns}$ \\
\hline
\end{tabular}

Table 2: Optimised parameters for laser ablation of holes on FR4.

\begin{tabular}{|l|l|}
\hline Power & $0.5 \mathrm{~W}$ \\
Energy & $62.5 \%$ \\
Frequency & $73 \mathrm{kHz}$ \\
No. of pulses per hole & 4 \\
\hline
\end{tabular}

The results of laser ablation on FR4 were encouraging, as holes with a diameter of about 10 $\mu \mathrm{m}$ and a depth of about $3.5 \mu \mathrm{m}$ were ablated. The profile of the holes was quite homogeneous as well. Figure 2(A) shows an FR4 substrate on which holes were ablated using the optimised parameter set listed in table 2. Figure 2(B) shows the cross-section of an ablated hole.
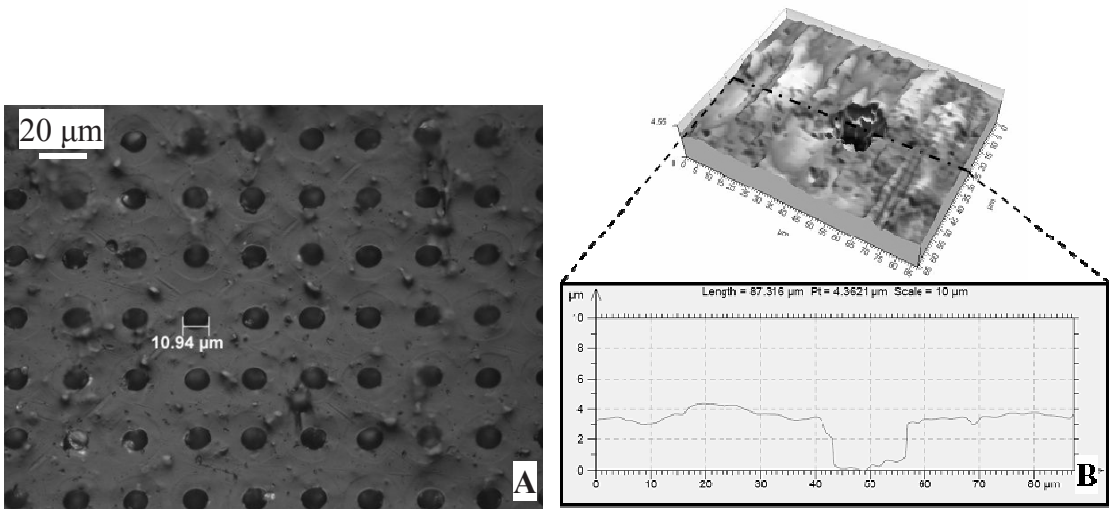

Figure 2: Laser ablated array of holes on FR4; (A) top-view using an optical microscope, and (B) cross-section of a single hole using a confocal scanning microscope. 
Appendix-B

\section{References}

[1] W.M. Cranton, S.L. Wilson, R. Ranson, D.C. Koutsogeorgis, K. Chi, R. Hedgley, J. Scott, S. Lipiec, A. Spiller and S. Speakman: "Excimer laser processing of inkjet-printed and sputter-deposited transparent conducting $\mathrm{SnO}_{2}: \mathrm{Sb}$ for flexible electronics", Thin Solid Films, Vol. 515, No. 24, p. 8534, 2007.

[2] S.H. Ko, H. Pan, C.P. Grigoropoulos, C.K. Luscombe, J.M.J. Frechet and D. Poulikakos: "All-inkjet-printed flexible electronics fabrication on a polymer substrate by low-temperature high-resolution selective laser sintering of metal nanoparticles", Nanotechnology, Vol. 18, No. 34, Doi: 10.1088/0957-4484/18/34/345202.

[3] M. Pudas, A. Kruusing, J. Saavalainen, J. Mahonen and J. Vahakangas: "Novel laser processing applications for microelectronics", Lasers in Engineering, Vol. 16, p. 133, 2006. [4] http://www.statsoft.com/textbook/statistics-glossary (accessed: February 2010). 


\section{Nomenclature}

\section{Latin}

$\begin{array}{lll}\text { Symbol } & \text { Expression } & \text { Unit } \\ \boldsymbol{A} & \text { Area } & \mathrm{m}^{2} \\ \boldsymbol{a} & \text { Contact radius } & \mathrm{m} \\ \boldsymbol{d} & \text { Depth } & \mathrm{m} \\ \boldsymbol{D} & \text { Diameter } & \mathrm{m} \\ \boldsymbol{E} & \text { Young's modulus } & \mathrm{N} \cdot \mathrm{m}^{-2} \\ \boldsymbol{f} & \text { Frequency } & \mathrm{Hz} \\ \boldsymbol{F} & \text { Force } & \mathrm{N} \\ \boldsymbol{G} & \text { Interfacial fracture energy } & \mathrm{J} . \mathrm{m}^{-2} \\ \boldsymbol{K} & \text { Bulk modulus } & \mathrm{N} \cdot \mathrm{m}^{-2} \\ \boldsymbol{n} & \text { Number } & - \\ \boldsymbol{p} & \text { Pitch } & \mathrm{m} \\ \boldsymbol{R}^{2} & \text { Coefficient of determination } & - \\ \boldsymbol{R}_{\boldsymbol{a}} & \text { Arithmetic average of profile surface roughness } & \mathrm{m} \\ \boldsymbol{S} & \text { Scattering parameters } & \mathrm{dB} \\ \boldsymbol{S}_{\boldsymbol{a}} & \text { Arithmetic average of areal surface roughness } & \mathrm{m} \\ \boldsymbol{S}_{\boldsymbol{S} L} & \text { Spreading coefficient } & - \\ \boldsymbol{t} & \text { Thickness } & \mathrm{m} \\ \boldsymbol{t} & \text { Time } & \mathrm{s} \\ \boldsymbol{T} & \text { Temperature } & { }^{\circ} \mathrm{C} \\ \boldsymbol{V} & \text { Voltage } & \mathrm{V} \\ \boldsymbol{Y} & \text { Yield } & \mathrm{m}, \mathrm{N} \cdot \mathrm{m}^{-1}\end{array}$


Nomenclature

Greek

Symbol Expression

Unit

$\boldsymbol{\alpha} \quad$ Level of significance

$-$

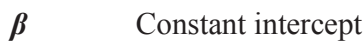

$\gamma \quad$ Surface free energy/Interfacial free energy/Surface tension $\quad \mathrm{J} . \mathrm{m}^{-2}, \mathrm{~N} . \mathrm{m}^{-1}$

$\boldsymbol{\delta} \quad$ Skin depth

$\mathrm{m}$

$\boldsymbol{\theta} \quad$ Contact angle

$\boldsymbol{\mu} \quad$ Permeability

H.m $\mathrm{m}^{-1}$ or $\Omega$. s. $\mathrm{m}^{-1}$

$v \quad$ Poisson's ratio

$\sigma \quad$ Electrical conductivity

$\boldsymbol{\sigma} \quad$ Tensile stress

$\Omega^{-1} \cdot m^{-1}$

$\mathrm{MPa}, \mathrm{N} \cdot \mathrm{m}^{-2}$ 
Abbreviations

\begin{tabular}{|c|c|}
\hline$A N O V A$ & ANalysis Of VAriance \\
\hline$C C R D$ & Central Composite Rotatable Design \\
\hline$D M A$ & Dynamic Mechanical Analysis \\
\hline DoE & Design of Experiments \\
\hline$E D T A$ & Ethylenediaminetetraacetic Acid \\
\hline FR4 & Flame Retardant 4 \\
\hline ISO & International Organization for Standardization \\
\hline IT & Information Technology \\
\hline LASER & Light Amplification by Stimulated Emission of Radiation \\
\hline$L E D$ & Light Emitting Diode \\
\hline MBST & Modified Button Shear Test \\
\hline NPS & Nanoparticle Silver \\
\hline$O L E D$ & Organic Light Emitting Diode \\
\hline$P A C M A N$ & Phased Array Communication Antennas for Mass-market Application Needs \\
\hline$P C B$ & Printed Circuit Board \\
\hline PEDOT:PSS & Poly(3,4-ethylenedioxythiophene) poly(styrenesulfonate) \\
\hline PRESS & Prediction Residual Error Sum of Squares \\
\hline$P V D$ & Physical Vapour Deposition \\
\hline$R F$ & Radio Frequency \\
\hline RFID & Radio Frequency Identification Device \\
\hline$R L C$ & Resistance-Inductance-Capacitance \\
\hline$R P M$ & Revolutions Per Minute \\
\hline SEM & Scanning Electron Microscope/Microscopy \\
\hline$S M A$ & Sub-Miniature Version A connector \\
\hline$S S$ & Sum of Squares \\
\hline $\boldsymbol{U} \boldsymbol{V}$ & Ultraviolet \\
\hline
\end{tabular}




\section{Summary}

A process chain to fabricate conductive structures on printed circuit board materials is presented in this thesis. This process chain comprises four main steps: plasma treatment, inkjet printing, electroless plating and functional characterisation. It represents a drastic reduction in the number of process steps when compared to the conventional lithography-based method, widely used in the printed circuit board industry.

The role of the process steps and how they are treated in this thesis can be summarised thus:

- Plasma treatment is done to render the surface of the polymeric substrate material suitable for inkjet printing, by modifying its surface morphology. The surface modification results in improved adhesion of the inkjet printed structure to the substrate. $A \mathrm{CF}_{4} / \mathrm{O}_{2}$ plasma treatment process is used in this thesis to modify the surface morphology. The influence of process parameters (viz. flowrates of gases, power and duration of plasma treatment) on a commercial printed circuit board material is studied in detail with the aid of design of experiments. Based on the design of experiments, conclusions concerning substrate surface morphology suitable for inkjet printing are arrived at.

- Inkjet printing, the core step of the process chain, is used to fabricate seed layers of the desired conductive circuit structures. For this purpose, two different ink types were investigated: a silver nanoparticle-based ink and an organic silver complex ink. The 3D build-up of structures by inkjet printing multiple layers is also studied in detail, even though it is not relevant for the proposed process chain.

- Electroless plating is done subsequent to inkjet printing, to deposit copper on the seed layers. The copper growth on silver leads to increased thickness of the conductive structures, resulting in increased electrical conductivity, besides improving the integrity of the structures. Another positive effect of copper plating is improved solderability, as soldering on inkjet printed silver structures resulted in local dissolution of silver.

- Mechanical and electrical characterisation represents the final step of the proposed process chain. Mechanical testing in the form of adhesion testing is 
Summary

performed using the Scotch tape test method and the pull-off test method. The influence of surface roughness on adhesion between printed silver and substrate is studied using the pull-off test method. Electrical characterisation is a standard procedure used in the industry to quantify the electrical performance of the fabricated circuit structures. In this thesis, return loss $\left(S_{11}\right)$, insertion loss $\left(S_{21}\right)$ and conductivity are the electrical parameters that are investigated.

The proposed process chain is validated by fabricating radio frequency circuit structures in the form of an S-band filter and a transmission line, and comparing their performance with that of conventionally fabricated S-band filter and transmission line. The comparison shows that the inkjet printing-electroless plating combination can be used even for the $\mathrm{GHz}$ frequency range, marking an improvement from the oft-discussed RFID applications in the context of inkjet printing. 


\section{Samenvatting}

In dit proefschrift wordt een produktieketen beschreven voor het maken van geleidende structuren op PCB-materialen (printed circuit board materialen).

Deze produktieketen bestaat in hoofdzaak uit vier stappen: een plasmabehandeling, inkjet printing, niet-elektrolytisch metalliseren en karakterisering van de functies. In vergelijking met de conventionele productiewijze op basis van lithografische processen, zoals nu gebruikelijk in de PCB industrie, betekent dit een drastische vermindering van het aantal benodigde processtappen.

- De plasmabehandeling wordt gedaan om de morfologie van het oppervlak van het polymere substraat te modificeren zodat het geschikt is voor inkjet printing. Het gevolg van deze oppervlakte modificatie is een verbeterde hechting van de door inkjet printing gemaakte structuur aan het substraat. Een $\mathrm{CF}_{4} / \mathrm{O}_{2}$ plasma is in dit werk gebruikt om het substraatoppervlak te modificeren. De invloed van de procesparameters (debiet van de gassen, vermogen en duur van de behandeling) is bestudeerd in het geval van een commercieel verkrijgbaar PCB materiaal. Op basis van een design of experiments worden conclusies getrokken aangaande de substraat oppervlaktemorfologie, geschikt voor inkjet printing.

- Inkjet printing is de wezenlijke stap in de productieketen en wordt toegepast om de gewenste geleidende sporen aan te brengen in de vorm van een laag kiemen. Voor dit doel werden twee typen inkt onderzocht: een inkt op basis van zilver in de vorm van nano-deeltjes, en een inkt op basis van een organisch zilvercomplex. Ook de ruimtelijke structuur die ontstaat bij inkjet printing in meerdere lagen is in detail onderzocht, hoewel dit niet direct relevant is voor de genoemde productieketen.

- In aansluiting op het inkjet printing stap is koper aangebracht op de kiemsporen door niet-elektrolytisch metalliseren. De aangroei van koper op de zilverkiemen leidt tot toename van de dikte van de geleidende structuren hetgeen resulteert in verbetering van de elektrische geleiding en verbetering van de robustheid van de structuren. Nog een positief effect van het aanbrengen van het koper is het 
Samenvatting

mogelijk maken van soldeerverbindingen; solderen op de zilverstructuren leidt tot het oplossen van het zilver in de soldeer.

- De laatste stap van de voorgestelde productieketen wordt gevormd door mechanische en electrische karakterisering. Mechanisch zijn de structuren getest door middel van de Scotch tape test en de pull-off test. De invloed van de oppervlakte ruwheid op de hechting tussen het geprinte zilver en het substraat is bestudeerd met behulp van de pull-off test. Electrische karakterisering is een standaard procedure in de industrie om de functionaliteit van de gefabriceerde circuits te meten. In dit werk worden de return loss $\left(S_{11}\right)$, de insertion loss $\left(S_{21}\right)$ en de electrische geleiding onderzocht.

De voorgestelde productieketen is gevalideerd door circuits te fabriceren die van toepassing zijn voor het radio-frekwentie gebied: een S-band filter en een transmissielijn, en hun eigenschappen te vergelijken met een conventioneel gemaakte Sband filter en transmissielijn. Deze vergelijking toont aan dat de combinatie van inkjet printing en niet-electrolytisch metalliseren zelfs bruikbaar is voor het $\mathrm{GHz}$ frekwentiegebied; hetgeen een verbetering betekent ten opzichte van de veel besproken RFID toepassing in samenhang met inkjet printing. 


\section{Acknowledgements}

'Man is a social animal' - this adage pretty much sums up what I would like to convey here. In the four-and-odd years I worked on my PhD at the University of Twente, I had the pleasure of interacting with a number of individuals from different professional and social backgrounds. All of them have one thing in common: they contributed constructively, directly or indirectly, to the manner in which my research as well as personality evolved. I hope to have had a similar effect on at least some of those individuals. I consider it a privilege to be able to give them due credit here.

Sir Isaac Newton remarked that he was able to see further as he stood on the shoulders of giants that preceded him. Now, I am not comparing myself with Newton, which will be tantamount to sacrilege; however, his conclusion is valid in my case too, as I was able to see further than I otherwise would have, by standing on the shoulders of my supervisor, Durk van Dijk, and my professor, Remko Akkerman. Durk, I am thankful for your invaluable scientific inputs, and the entertaining chit-chats on history and current affairs. I will surely miss our discussions on a wide range of topics, as far afield as the Gothic Architecture and the Dutch Golden Age. Remko, many thanks for periodically reminding me of the big picture. In spite of your high standing in academia, your down-to-earth demeanour and amiable personality even in the face of difficulties will be something I will always remember and try to emulate.

Thanks are due to my project-mates at Thales Nederland B.V. and ASTRON. I would like to make a special mention of the contributions of Jan Mannak, Rob Legtenberg and Bernard Enthoven. Jan: for your insight in bridging academic research with industrial requirements, and for your efforts in finding competent students (Jacob, Mattijs, Terrence and Olav) who made valuable contributions to my thesis. Rob: for your technical inputs, and for meticulously reading my thesis and giving me valuable feedback. Bernard: for your constant interest in the progress of my research.

Quite logically, a major chunk of my time in the past few years was spent at the university (even though Laurent 'Gandhi' Warnet will disagree with me), with my colleagues - and what a time it was! I consider myself lucky to have interacted with 
Acknowledgements

them on a daily basis. Rene: thanks for your encouraging words when things were not really going as planned at the beginning of my research, and for being the ideal officemate. Dimitris: thanks for sharing your expertise in specimen preparation and polishing, for the squash games, and for agreeing to be my paranymph. Wouter: thanks for the entertaining squash and chess games, and for your critical review of my thesis. Sebastiaan: thanks for your valuable inputs on my thesis - you and Wouter raised so many questions about the contents, which, despite the extra work, resulted in a definite improvement of my thesis. Laurent: thanks for 'flanzing' the peel test setup that I under-utilised. Laura: thanks for the nice SEM images and for promptly arranging the required things in the lab. Ton, Bert, Gert Jan, Erik van de Ven and Michael de Groot: thanks for your inputs/assistance at various stages of my research. Erik de Vries and Walter Lette: a special mention for your support in carrying out experiments, despite not being involved in my project. Wessel: I enjoyed our discussions concerning the possibilities of inkjet printing. Debbie, Belinda and Tanja: thanks for promptly taking care of the administrative formalities.

Jolke Perelaer, thank you for the pleasant professional and casual interactions we have had in the past four years; an additional thanks to you, along with Patrick Smith and Prof. Schubert, for facilitating my initiation into inkjet printing. George Wheadon, thank you for arranging different types of substrate materials with enthusiasm.

Perhaps the most important outcome of my stay at the University of Twente is the number of friends I made. For want of space, I refrain from listing each and every one of them. But that does not diminish their importance by any means.

Sandeep, Jithu, Vishnu, Srivatsa and Pramod: you deserve a special mention for the typical friendly Indian interactions, food, get-together etc, and Pelagia, for being a good friend and for agreeing to be my paranymph; I am glad to have two Greeks as my paranymphs, thus honouring the Greek tradition.

My parents deserve a very special mention for all the hard work they have put in over the years, as well as for their support and confidence in me. 
Finally, I dedicate this thesis to my wife Sowmya: it is not an exaggeration to say that I would not have come this far without her support, motivation, understanding, enthusiasm and love. 


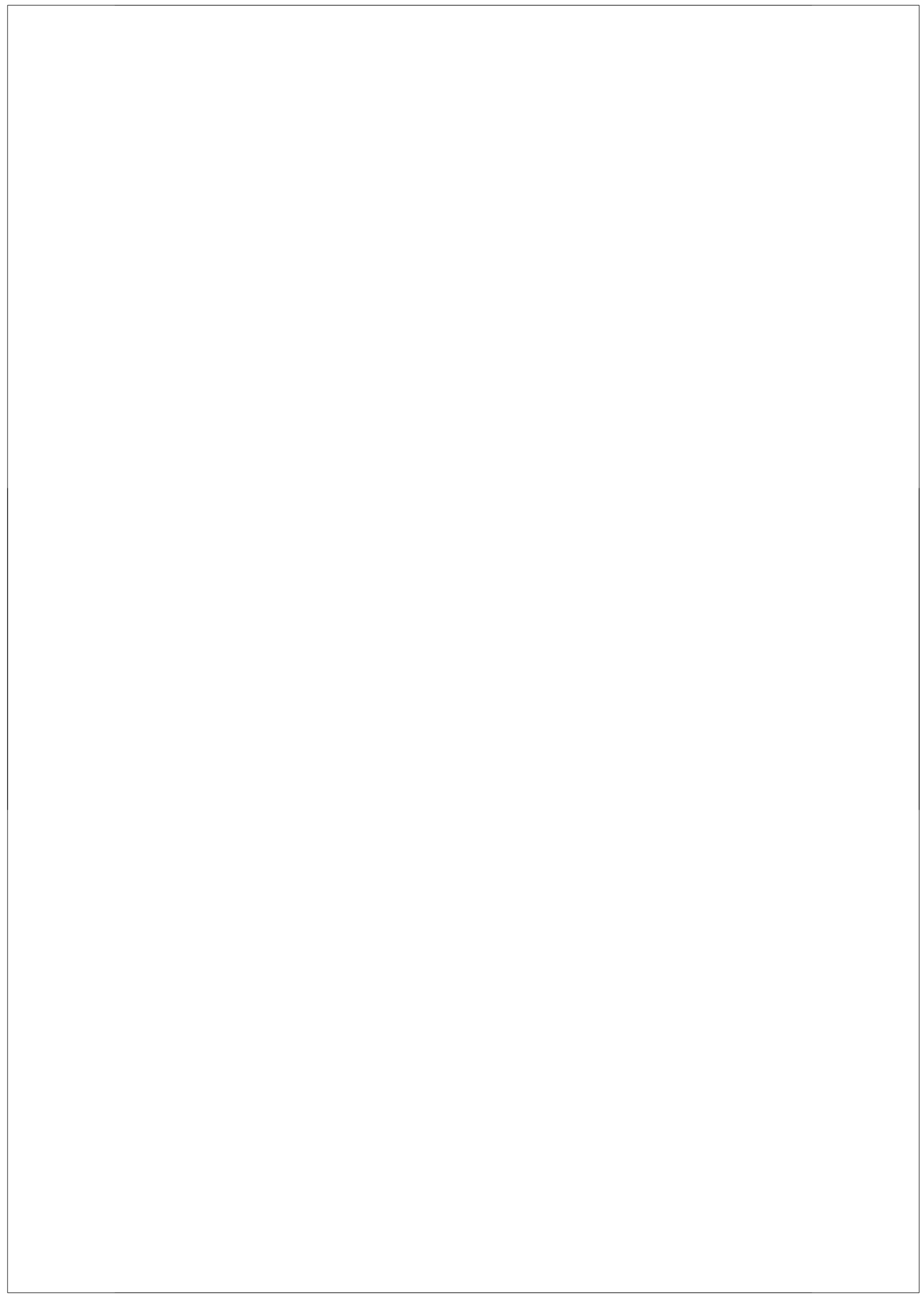

